\title{
Quantifying Solvophobic Effects in Organic Solvents using a Hydrocarbon Molecular Balance
}

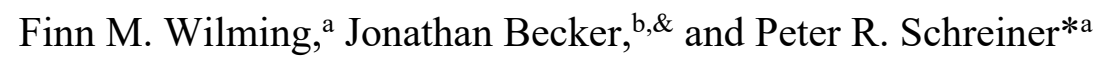

a Institute of Organic Chemistry, Justus Liebig University, Heinrich-Buff-Ring 17, 35392

Giessen (Germany) and Center for Materials Research (ZfM), Justus Liebig University,

Heinrich-Buff-Ring 16, 35392 Giessen (Germany)

${ }^{b}$ Institute of Inorganic and Analytical Chemistry, Justus Liebig University, Heinrich-Buff-

Ring 17, 35392 Giessen (Germany)

${ }^{\&}$ X-ray crystal structure determination

*Email:prs@uni-giessen.de

\section{Table of Contents}

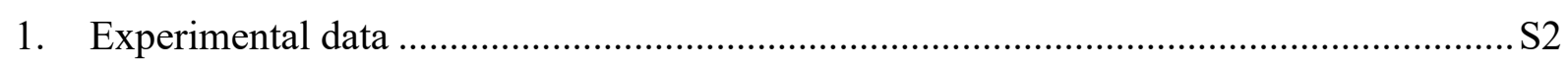

1.1. General procedure for obtaining $\Delta G_{Z / E}$ from NMR data ................................... S2

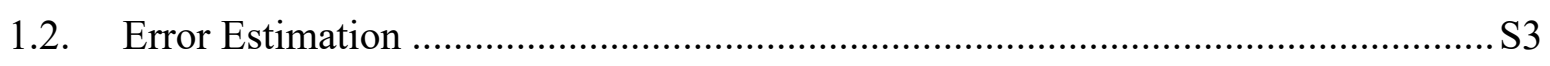

1.3. Influence of dipole moment on the equilibrium between $\mathrm{S} 1 E / Z$........................... S4

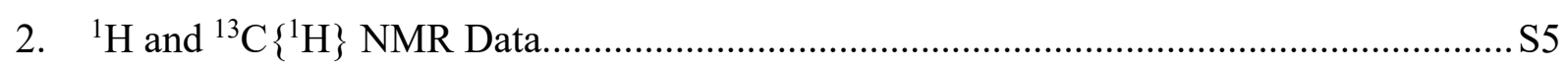

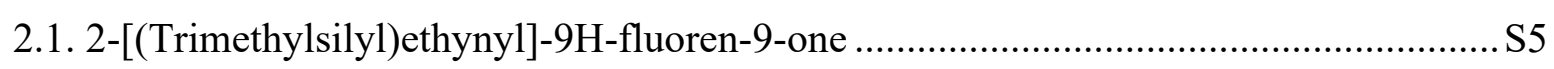

2.2. 2,2'-Bis[(trimethylsilyl)ethynyl]-9,9'-bifluorenylidene ............................................. S6

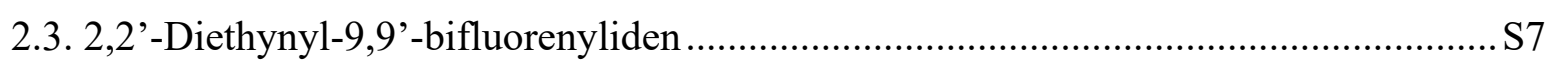

3. High Resolution Mass Spectrometry Data............................................................... S8

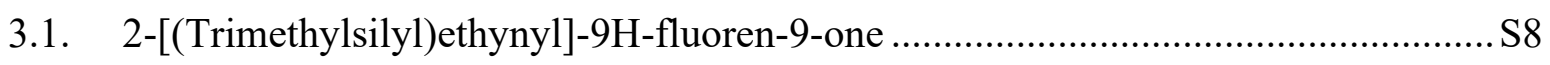

3.2. 2,2'-Bis[(trimethylsilyl)ethynyl]-9,9'-bifluorenylidene ......................................... S10

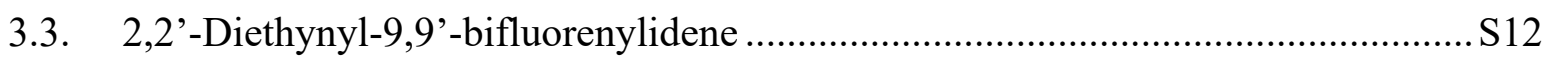

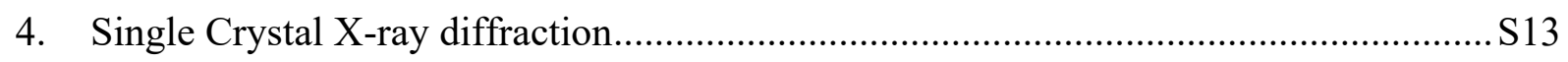

4.1. (1Z) Z-2,2'-Diethynyl-9,9'-bifluorenylidene........................................................... S14

4.2. (1E) E-2,2'-Diethynyl-9,9'-bifluorenylidene ....................................................... S25

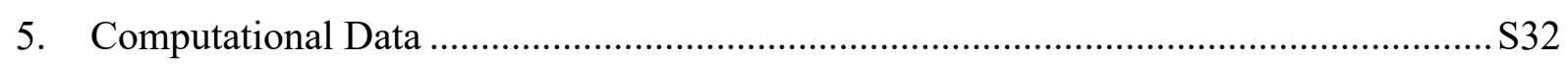

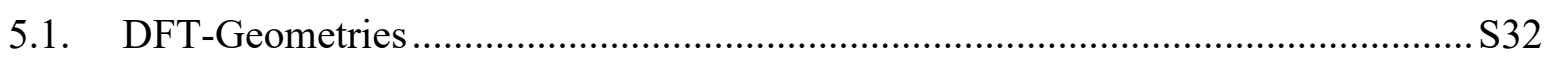

5.2. Symmetry Adapted Perturbation Theory (SAPT) of the Dimers ........................... S34

5.3. SMD Solvent Model Computations ……........................................................... S35 
5.3.1. E-2,2'-Diethynyl-9,9'-bifluorenylidene S35

5.3.2. Z-2,2'-Diethynyl-9,9'-bifluorenylidene S43

5.3.3. E-3,3'-Dibromo-9,9'-bifluorenylidene S51

5.3.4. Z-3,3'-Dibromo-9,9'-bifluorenylidene S53

6. References S55

\section{Experimental data}

\subsection{General procedure for obtaining $\Delta G_{Z / E}$ from NMR data}

All NMR-measurements for the determination of $\Delta G_{Z / E}$ were carried out with a Bruker Avance III HD $600 \mathrm{MHz}$ spectrometer heated to $333 \mathrm{~K}$. We used the following deuterated solvents due to their high boiling point and commercial availability:

acetic acid- $d_{4}$, benzene- $d_{6}$, bromobenzene- $d_{5}$, chlorobenzene- $d_{5}$, cyclohexane- $d_{12}, n$-dodecane$d_{26}$, ethanol- $d_{6}$, methanol- $d_{4}$, nitrobenzene- $d_{5}, \quad n$-octane- $d 18$, pyridine- $d_{5}, \quad 1,1,2,2-$ tetrachloroethane- $d_{2}$, THF- $d_{8}$, toluene- $d_{8}, p$-xylene- $d_{10}$.

All NMR spectra were interpreted using MestReNova version 14.2.0-26256. After polynomial fit baseline correction for all obtained ${ }^{1} \mathrm{H}-\mathrm{NMR}$ spectra, we used the line fitting option with the generalized Lorentzian shape type to integrate the signals of the protons in 1, 1', 8 , and 8' position of the bifluorenylidene backbone. The obtained values for $K_{Z / E}$ together with the calculated values for $\Delta G_{Z / E}$ are shown in Table S1.

Table S1. Experimentally obtained values for $K_{E / Z}$ and $\Delta G_{E / Z \text {. }}$

\begin{tabular}{|ccc|}
\hline Solvent & $\boldsymbol{K}_{\boldsymbol{Z} / \boldsymbol{E}}$ & $\Delta \boldsymbol{G}_{\boldsymbol{Z} / \boldsymbol{E}} / \mathbf{~ k c a l ~}^{\mathbf{~ m o l}}{ }^{\mathbf{1}}$ \\
\hline cyclohexane- $d_{12}$ & 0.729 & 0.20 \\
\hline toluene- $d_{8}$ & 0.732 & 0.20 \\
\hline$p$-xylene- $d_{10}$ & 0.747 & 0.19 \\
\hline$n$-dodecane- $d_{26}$ & 0.754 & 0.18 \\
\hline $1,1,2,2$-tetrachloroethane- $d_{2}$ & 0.755 & 0.18 \\
\hline$n$-octane- $d_{18}$ & 0.763 & 0.17 \\
\hline benzene- $d_{6}$ & 0.764 & 0.17 \\
\hline bromobenzene- $d_{5}$ & 0.773 & 0.17 \\
\hline THF- $d_{8}$ & 0.778 & 0.16 \\
\hline chlorobenzene- $d_{5}$ & 0.781 & 0.16 \\
\hline pyridine- $d_{5}$ & 0.787 & 0.15 \\
\hline nitrobenzene- $d_{5}$ & 0.819 & 0.13 \\
\hline ethanol- $d_{6}$ & 0.830 & 0.12 \\
\hline acetic acid- $d_{4}$ & 0.869 & 0.09 \\
\hline methanol- $d_{4}$ & 0.920 & 0.05 \\
\hline
\end{tabular}

We calculated the ced of the used solvents at $333 \mathrm{~K}$ using [S1+S2]. The experimental values for $\Delta H_{\mathrm{v}}$ and $V_{\mathrm{m}}$ together with the obtained results are listed in Table $\mathrm{S} 2$. 


$$
\begin{gathered}
\text { ced }=\frac{\Delta H_{v}-R T}{V_{m}} \\
V_{m}=\frac{M}{\rho}
\end{gathered}
$$

Table S2. Solvent properties used to calculate the ced at $333 \mathrm{~K}$. We chose $\Delta H_{v}$ values from literature that were measured closest to $333 \mathrm{~K}$. We used the density at r.t. because of unavailability of temperature dependent density

\begin{tabular}{|c|c|c|c|c|c|}
\hline Solvent & $\begin{array}{c}\Delta H_{\mathrm{v}} / \\
\mathrm{cal} \mathrm{mol}^{-1}\end{array}$ & 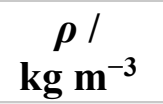 & $\begin{array}{c}M / \\
\mathrm{g} \mathrm{mol}^{-1}\end{array}$ & $\begin{array}{c}V \mathbf{m} / \\
\mathbf{c m}^{3} \mathbf{m o l}^{-1}\end{array}$ & $\begin{array}{c}\text { ced / } \\
\text { cal } \mathbf{c m}^{-3}\end{array}$ \\
\hline cyclohexane- $d_{12}$ & $7452^{[1]}$ & 893 & 96.23 & 107.8 & 43.5 \\
\hline toluene- $d_{8}$ & $8455^{[2]}$ & 943 & 100.19 & 106.2 & 53.5 \\
\hline$p$-xylene- $d_{10}$ & $9936^{[3]}$ & 948 & 116.09 & 122.5 & 56.0 \\
\hline$n$-dodecane- $d_{26}$ & $13877^{[4]}$ & 864 & 196.5 & 227.4 & 48.8 \\
\hline $\begin{array}{c}1,1,2,2- \\
\text { tetrachloroethane- } d_{2}\end{array}$ & $10008^{[5]}$ & 1620 & 169.86 & 104.9 & 69.0 \\
\hline$n$-octane- $d_{18}$ & $9339^{[1]}$ & 815 & 132.34 & 162.4 & 40.5 \\
\hline benzene- $d_{6}$ & $7619^{[6]}$ & 950 & 84.15 & 88.6 & 54.8 \\
\hline bromobenzene- $d_{5}$ & $10127^{[7]}$ & 1539 & 162.05 & 105.3 & 69.9 \\
\hline THF $-d_{8}$ & $7356^{[8]}$ & 985 & 80.16 & 81.4 & 56.4 \\
\hline chlorobenzene- $d_{5}$ & $9267^{[5]}$ & 1157 & 117.59 & 101.6 & 63.9 \\
\hline pyridine- $d_{5}$ & $9196^{[9]}$ & 1050 & 84.13 & 80.1 & 80.2 \\
\hline nitrobenzene- $d_{5}$ & $12969^{[10]}$ & 1253 & 128.14 & 102.3 & 99.7 \\
\hline ethanol- $d_{6}$ & $9602^{[6]}$ & 892 & 52.11 & 58.4 & 117.0 \\
\hline acetic acid- $d_{4}$ & $9769^{[11]}$ & 1119 & 64.08 & 57.3 & 122.2 \\
\hline methanol- $d_{4}$ & $8957^{[12]}$ & 888 & 36.07 & 40.6 & 152.4 \\
\hline
\end{tabular}
data for deuterated solvents.

\subsection{Error Estimation}

For the estimation of experimental error, we used the total differential of [S3] derived in [S4] $-[\mathrm{S} 6]$. We estimated the error of quantitative NMR analysis to be $5 \% . \Delta T=0.15 \mathrm{~K}$.

$$
\begin{gathered}
\Delta G_{Z / E}=-\ln \left(K_{Z / E}\right) \cdot R \cdot T \\
\Delta \Delta G_{Z / E}=\frac{\partial\left(-\ln \left(K_{Z / E}\right) \cdot R \cdot T\right)}{\partial K_{Z / E} \cdot T} \cdot \Delta K_{\text {fold }} \cdot \Delta T \\
K_{Z / E}=\frac{c[\text { folded }]}{c[\text { unfolded }]} \sim \frac{\int A[\text { folded }]}{\int A[\text { unfolded }]} \\
\Delta \Delta G_{Z / E}=\left|-\frac{R \cdot \ln \int A[\text { unfolded }] \cdot T}{\int A[\text { folded }]}\right| \cdot \Delta \int A[\text { folded }]+ \\
\left|-\frac{R \cdot \ln \int A[\text { folded }] \cdot T}{-\int A[\text { unfolded }]}\right| \cdot \Delta \int A[\text { unfolded }]+
\end{gathered}
$$




$$
\left|-\ln \frac{\int A[\text { folded }]}{\int A[\text { unfolded }]} \cdot R\right| \cdot \Delta T
$$

We calculated an estimated error of $0.01 \mathrm{kcal} \mathrm{mol}^{-1}$ for all 15 measurements.

\subsection{Influence of dipole moment on the equilibrium between $\mathrm{S} 1 E / Z$}

To probe the influence of the difference in dipole moments of the $E$ - and $Z$-isomers on the $E / Z$ equilibrium of $\mathbf{1 E} / \boldsymbol{Z}$ we synthesized 3,3'-dibromo-9,9'-bifluorenylidene $\mathbf{S} \boldsymbol{E} / \boldsymbol{Z}$ according to literature procedures and experimentally determined $E / Z$ ratios in solvents of varying polarity. ${ }^{[13]}$ We chose this system because its bromine substituents are positioned such that they are expected have no noncovalent interactions with each other and the computed dipole moments (B3LYP-D3/def2SVP; Figure S1) are comparable to $\boldsymbol{1} \boldsymbol{E} / \boldsymbol{Z}$ (Table S3). We also computed the sas of both isomers to be almost identical $\left( \pm 0.2 \AA^{2}\right)$ which excludes the possibility of solvophobic effects influencing the equilibrium between $\mathbf{S 1 E}$ and $\mathbf{S 1 Z}$.
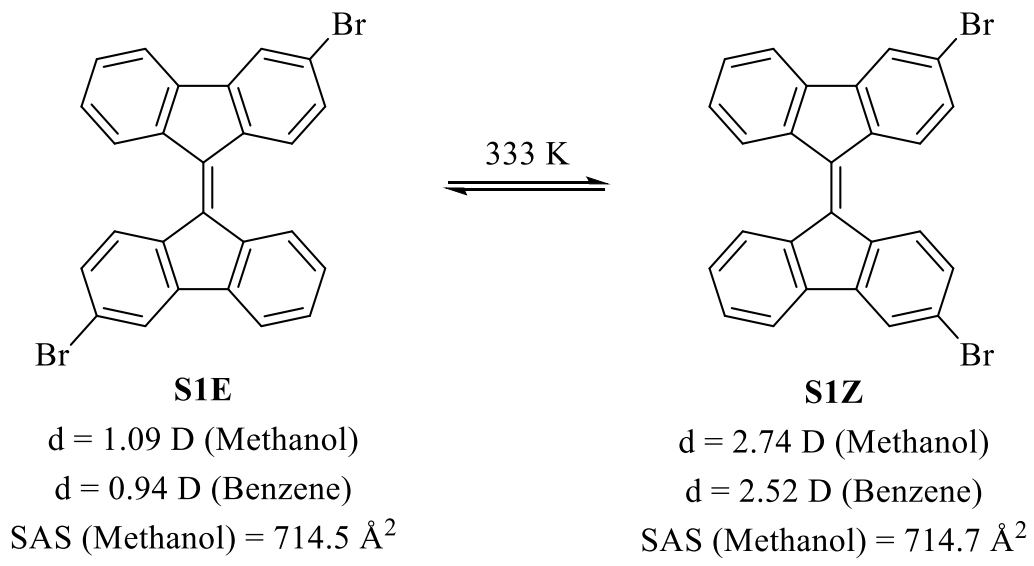

Figure S1. We used the equilibrium between $\mathbf{S 1 E}$ and $\mathbf{S 1 Z}$ to probe the influence of difference in dipole moment of the two isomers on the E/Z ratios in organic solvents with varying polarity.

We measured $\Delta G_{E / Z}$ in $p$-xylene- $d_{10}$, benzene- $d_{6}$, chlorobenzene- $d_{5}$ and bromobenzene- $d_{5}$ and found a $Z$-favorization by only $0.01 \mathrm{kcal} \mathrm{mol}^{-1}$ for all four solvents. The equilibrium between $\mathbf{S} 1 \boldsymbol{E}$ and $\mathbf{S} 1 Z$ therefore seems to be almost uninfluenced by a change in solvent polarity. We chose aromatic solvents with differing dipole moments because the solubility of $\mathbf{S} \boldsymbol{E} / \boldsymbol{Z}$ is very limited in non-aromatic solvents. Transferring these findings to the molecular balance system of $1 E / Z$, they suggest that a difference in dipolemoment of $1 E / Z$ is generally not much affecting the $E / Z$-ratios in solvents with different polarity.

Table S3. Dipole moments of $1 E / Z$ computed at B3LYP-D3/def2SVP

\begin{tabular}{|ccc|}
\hline Solvent & $\overrightarrow{\boldsymbol{d}}(\mathbf{1 E}) /$ Debye & $\overrightarrow{\boldsymbol{d}}(\mathbf{1 Z}) /$ Debye \\
\hline cyclohexane- $d_{12}$ & 0.75 & 1.65 \\
\hline toluene- $d_{8}$ & 0.76 & 1.67 \\
\hline benzene- $d_{6}$ & 0.76 & 1.67 \\
\hline THF- $d_{8}$ & 0.79 & 1.78 \\
\hline chlorobenzene- $d_{5}$ & 0.83 & 1.74 \\
\hline ethanol- $d_{6}$ & 0.82 & 1.89 \\
\hline methanol- $d_{4}$ & 0.84 & 1.96 \\
\hline
\end{tabular}




\section{2. ${ }^{1} \mathrm{H}$ and ${ }^{13} \mathrm{C}\left\{{ }^{1} \mathrm{H}\right\}$ NMR Data}

\subsection{2-[(Trimethylsilyl)ethynyl]-9H-fluoren-9-one}

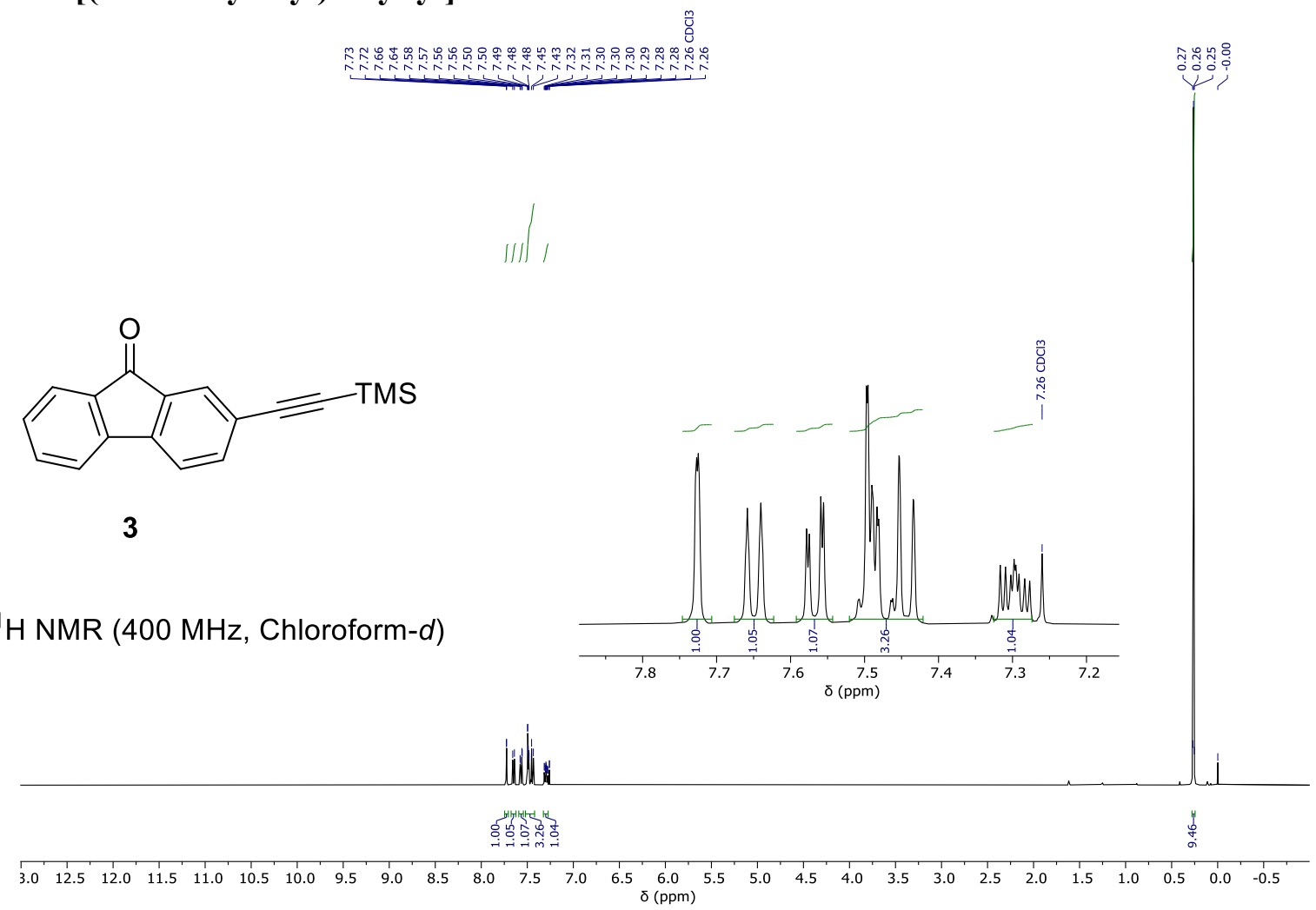

Figure S2. ${ }^{1} H$-NMR spectrum for 3 measured in Chloroform-d.

\begin{tabular}{|c|c|c|c|}
\hline & 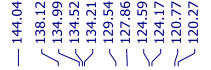 & 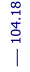 & 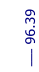 \\
\hline
\end{tabular}

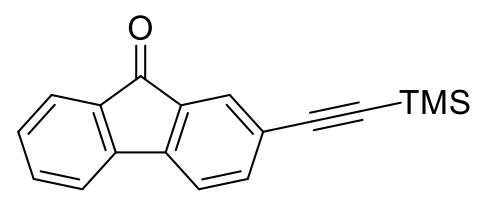

3

${ }^{13} \mathrm{C}\left\{{ }^{1} \mathrm{H}\right\}$ NMR $(100 \mathrm{MHz}$, Chloroform- $d)$

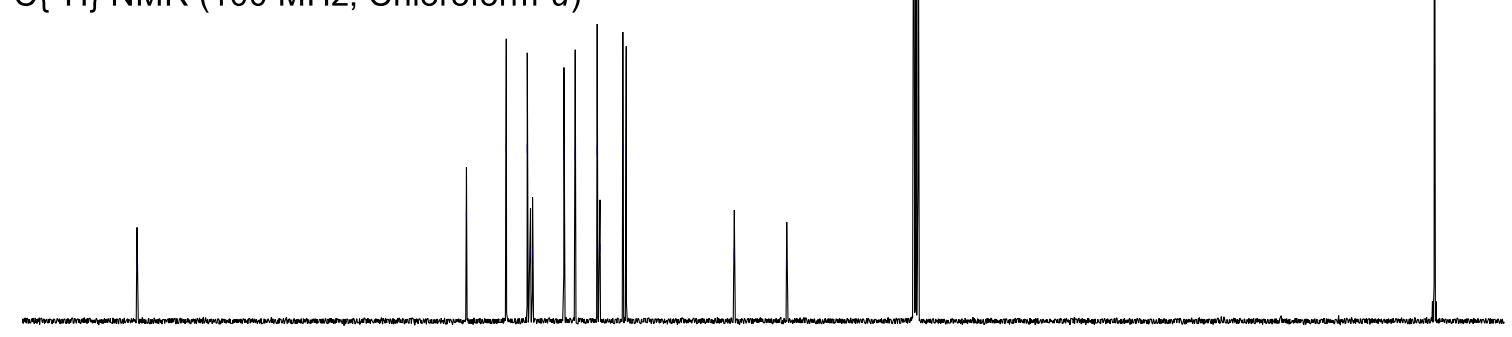

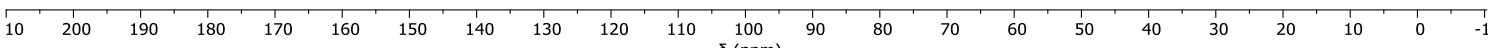

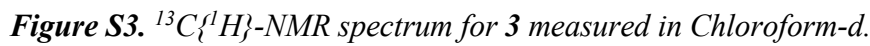




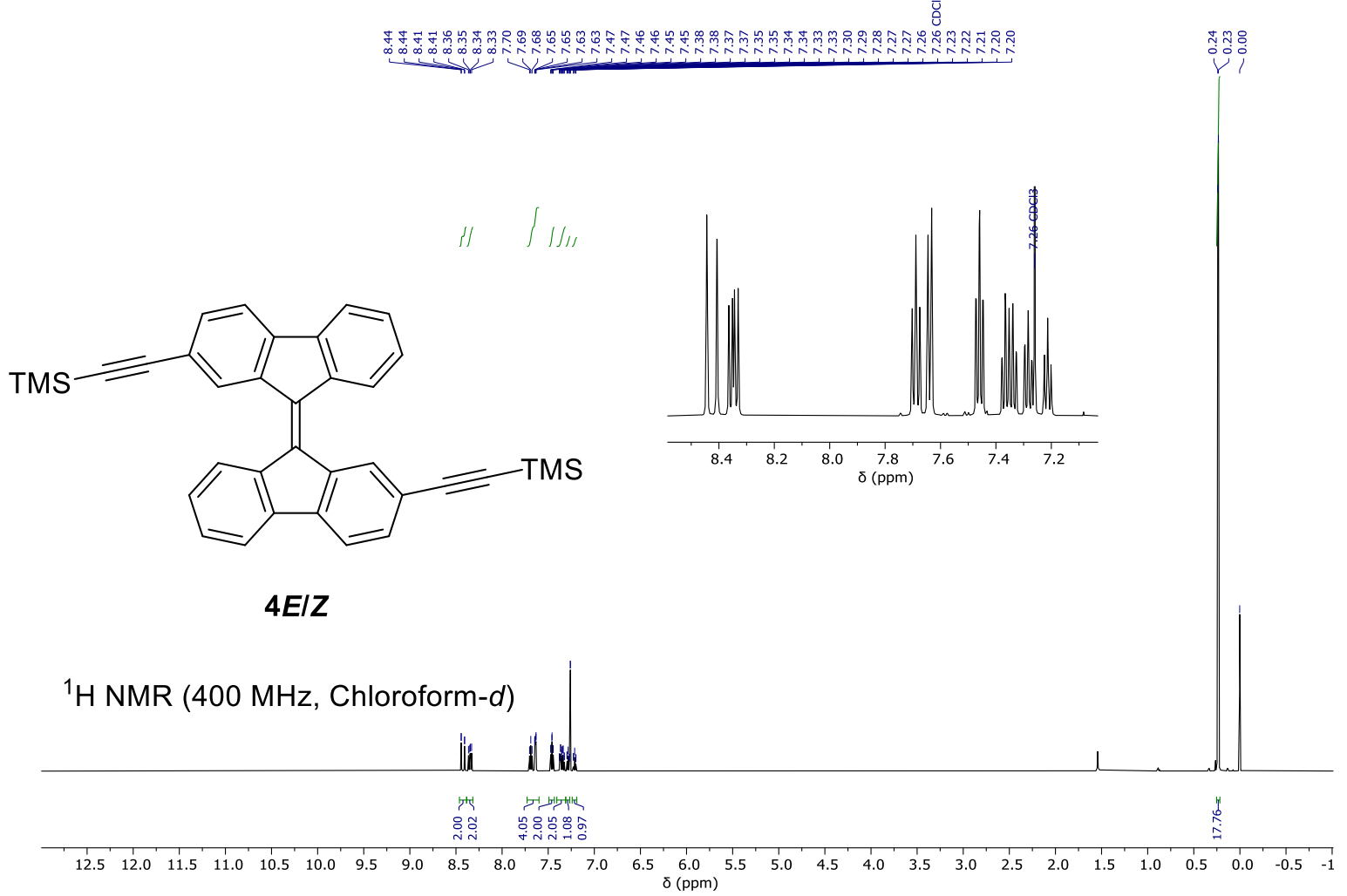

Figure S4. ${ }^{1} H$-NMR spectrum for $4 \boldsymbol{E} / \mathbf{Z}$ measured in Chloroform-d.

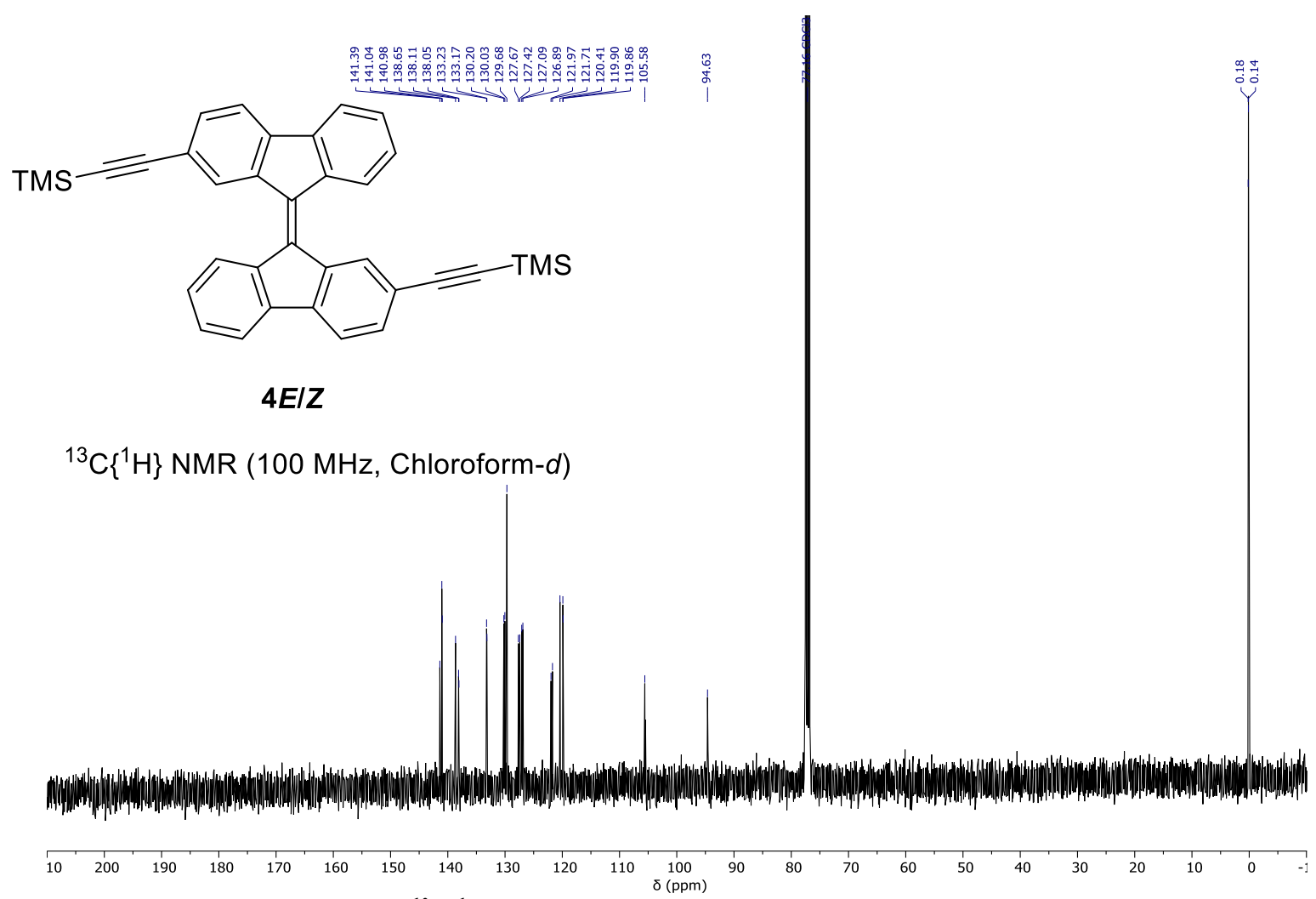

Figure S5. ${ }^{13} C\left\{{ }^{1} H\right\}-N M R$ spectrum for $\mathbf{4 E} / \boldsymbol{Z}$ measured in Chloroform-d. 


\subsection{2,2'-Diethynyl-9,9'-bifluorenyliden}

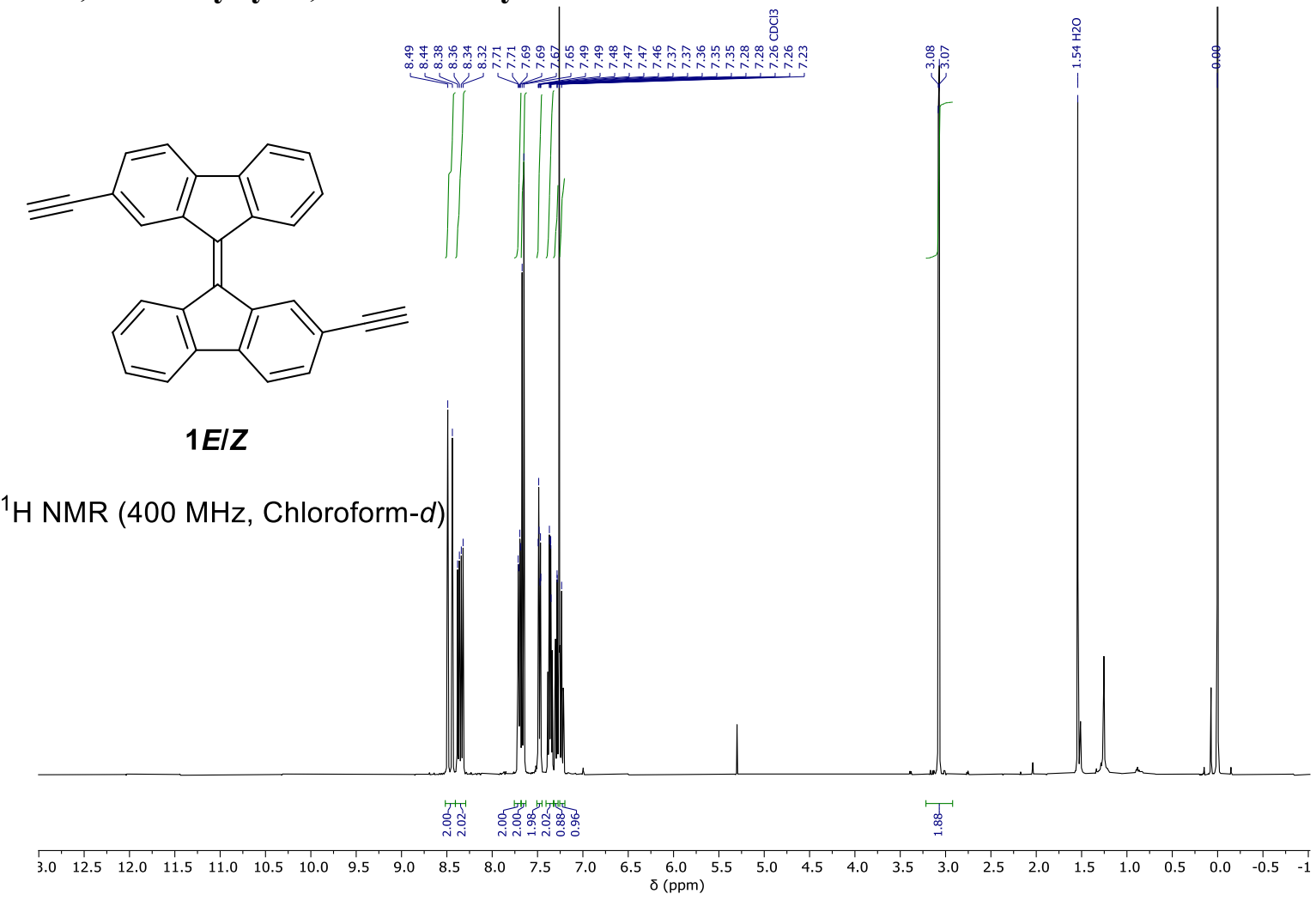

Figure S6. ${ }^{1} H$-NMR spectrum for $\mathbf{1 E} / \mathbf{Z}$ measured in Chloroform-d.

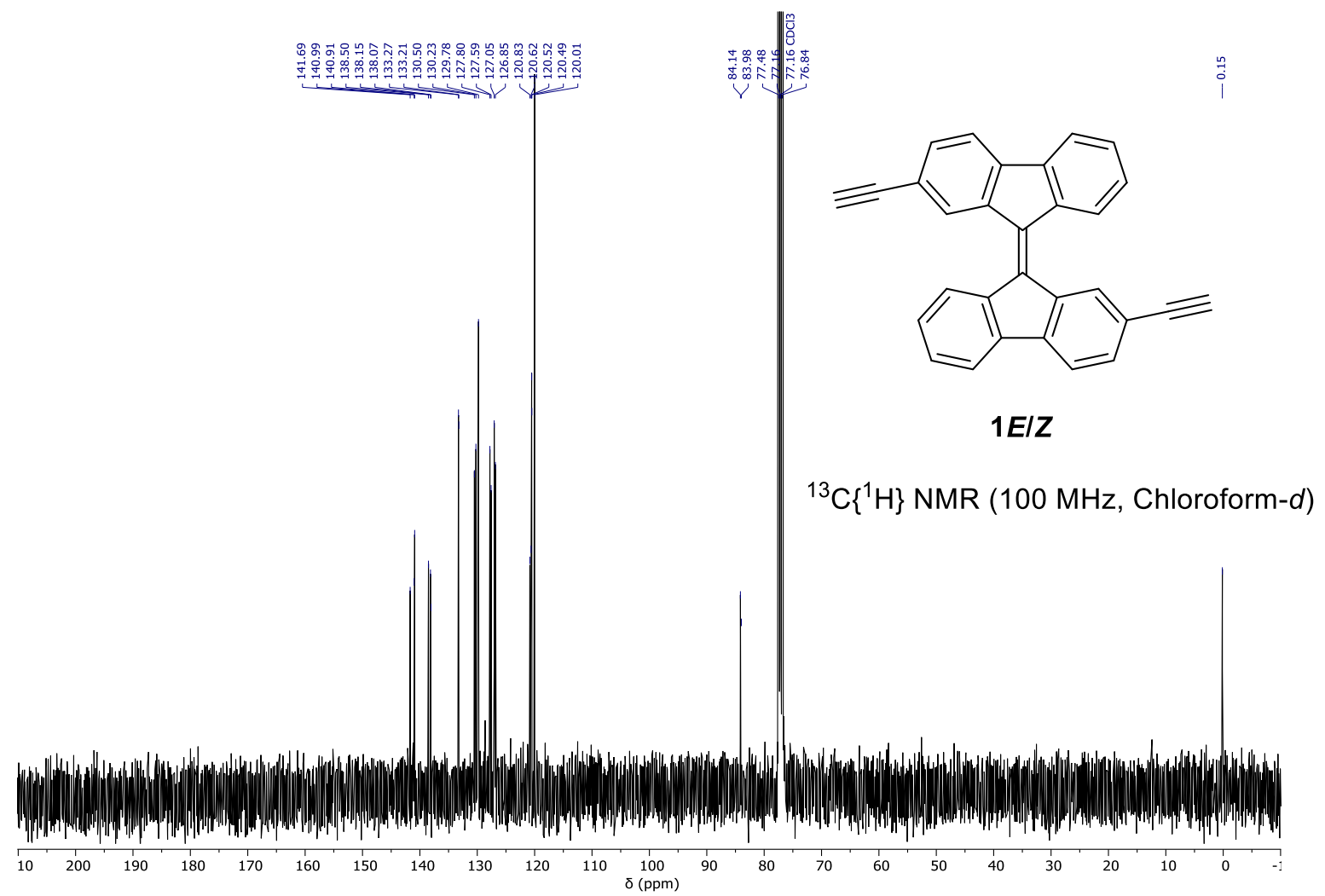

Figure S7. ${ }^{13} C\left\{{ }^{1} H\right\}$-NMR spectrum for $\mathbf{1 E} / \mathbf{Z}$ measured in Chloroform-d. 
3. High Resolution Mass Spectrometry Data

3.1. 2-[(Trimethylsilyl)ethynyl]-9H-fluoren-9-one

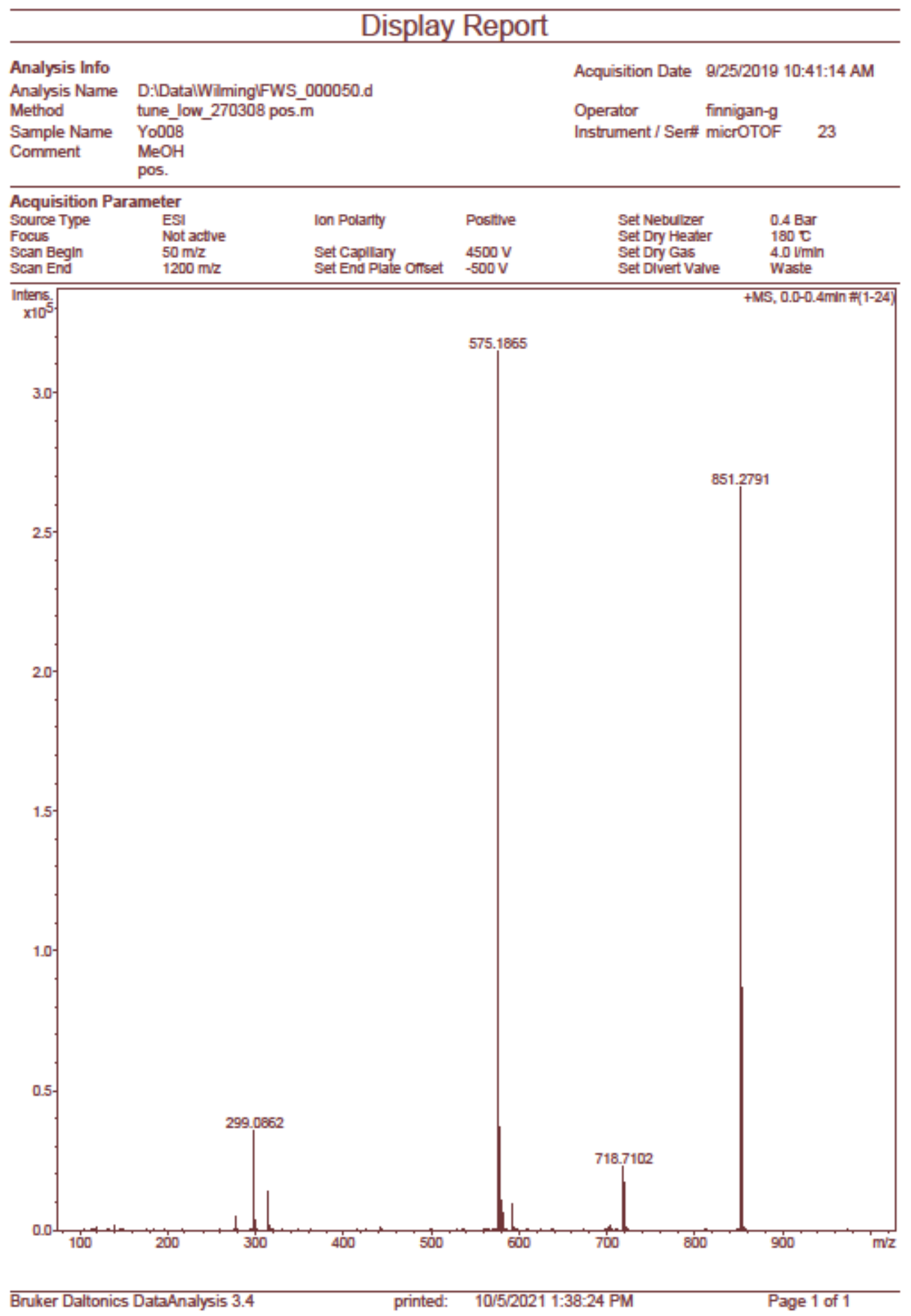

Figure S8. HRMS-report for 3. 


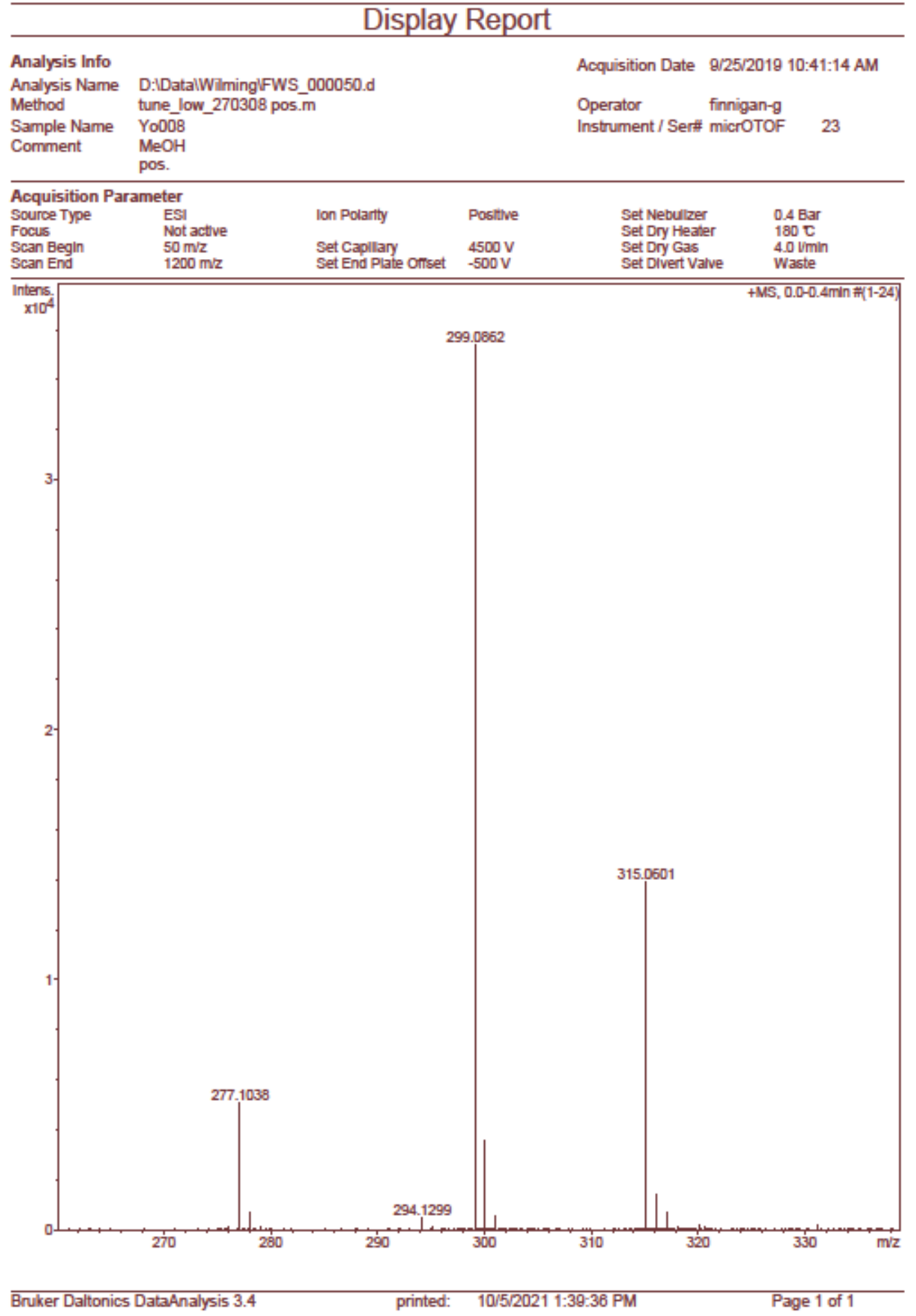

Figure S9. HRMS-report for 3. 


\subsection{2,2'-Bis [(trimethylsilyl)ethynyl]-9,9'-bifluorenylidene}

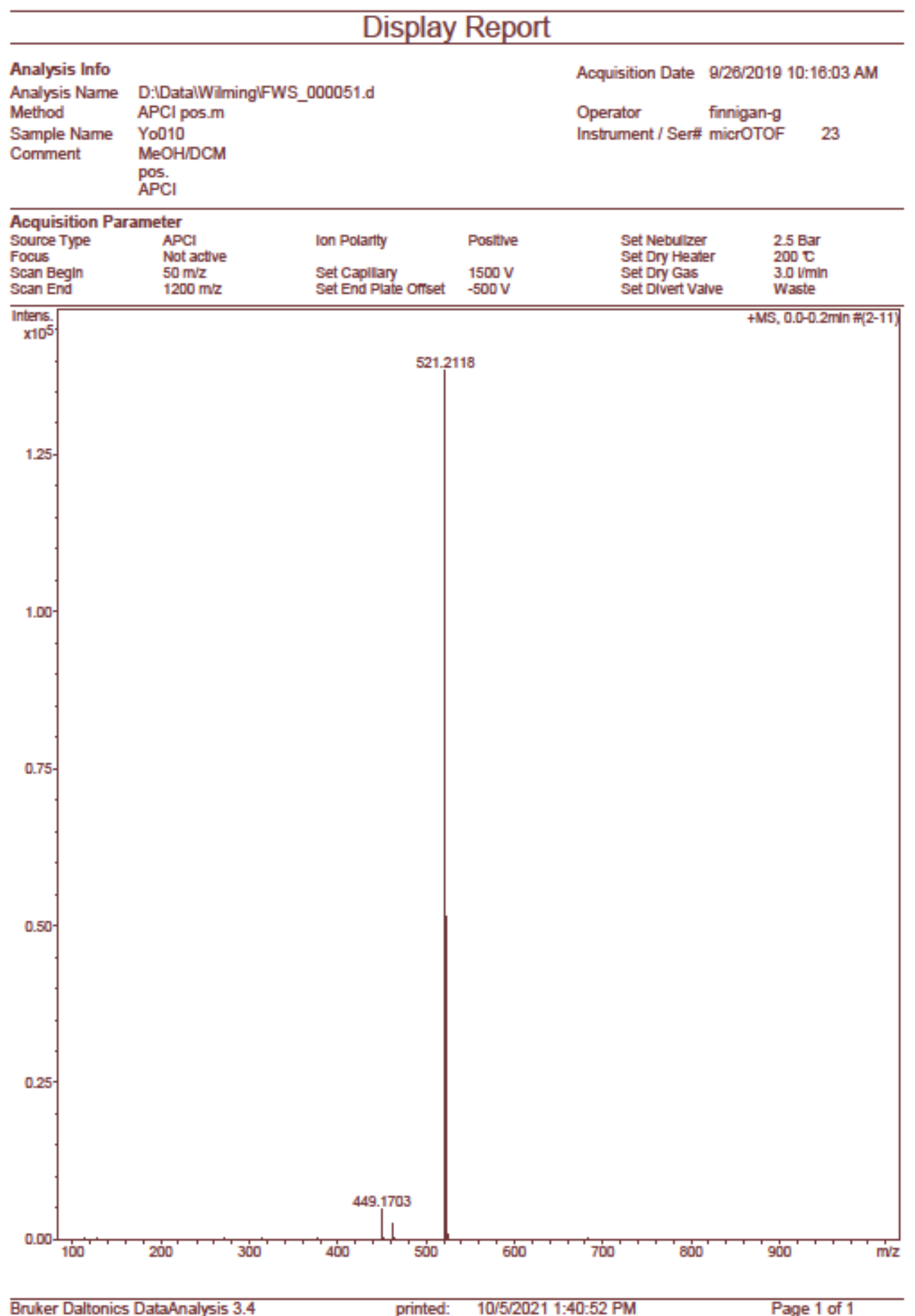

Figure S10. HRMS-report for $4 E / Z$. 


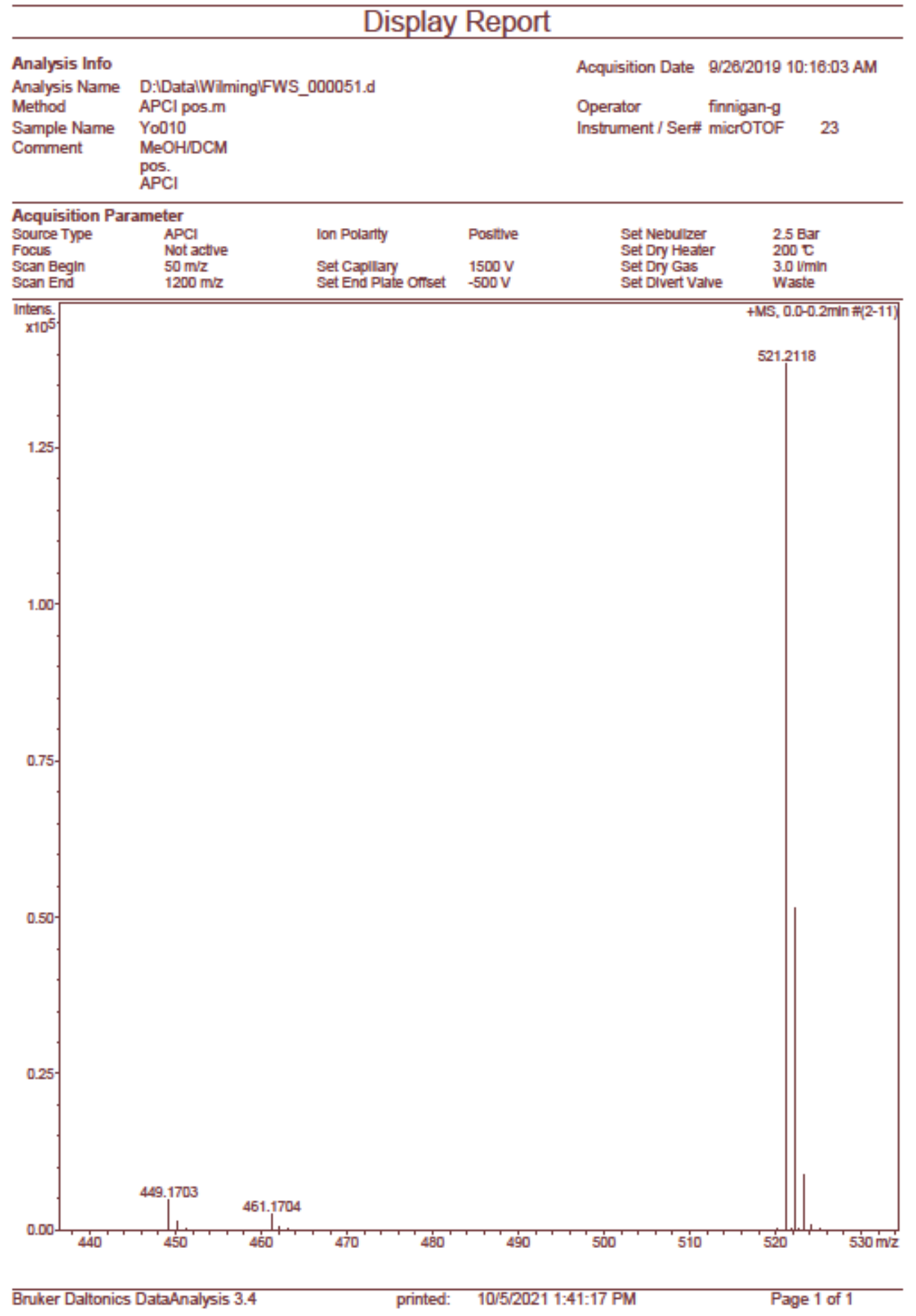

Figure S11. HRMS-report for $4 E / Z$ 
3.3. 2,2'-Diethynyl-9,9'-bifluorenylidene

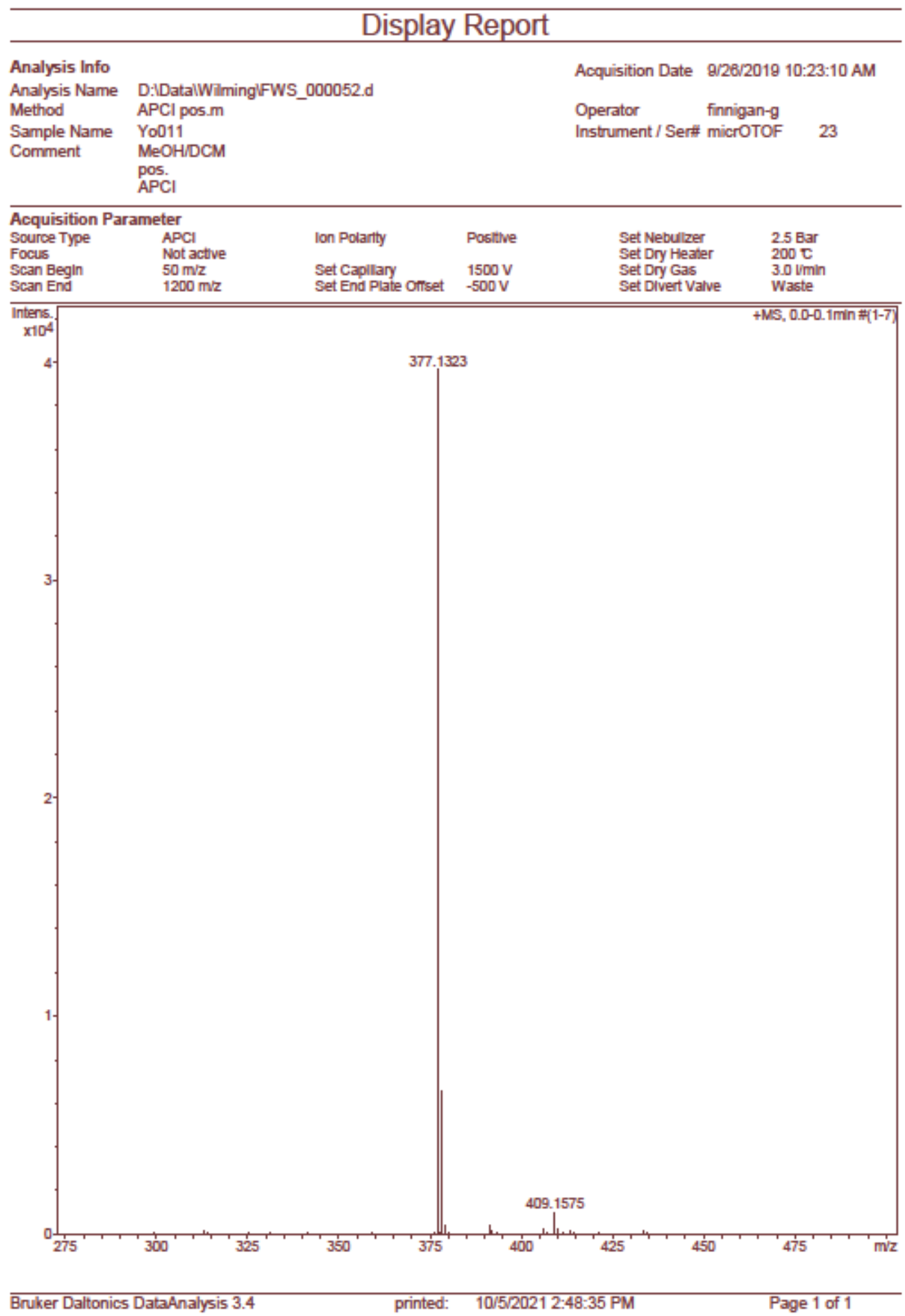

Figure S13. HRMS-report for $1 \boldsymbol{E} / \boldsymbol{Z}$. 


\section{Single Crystal X-ray diffraction}

Crystals were obtained after column chromatography of $\mathbf{1} \boldsymbol{E} / \boldsymbol{Z}$ with $n$-hexane/DCM (85:15) and subsequent evaporation of the solvent from the test tubes.

Diffraction data for $\mathbf{1 E}$ and $\mathbf{1 Z}$ were collected at low temperatures $(100 \mathrm{~K})$ using $\varphi$ - and $\omega$-scans on a BRUKER D8 Venture system equipped with dual I $\mu \mathrm{S}$ microfocus sources, a PHOTON100 detector and an OXFORD CRYOSYSTEMS 700 low temperature system. Mo- $\mathrm{K}_{\alpha}$ radiation with wavelength $0.71073 \AA$ and a collimating Quazar multilayer mirror were used. Semiempirical absorption correction from equivalents was applied using SADABS-2016/2 ${ }^{[14]}$ and the structures were solved by direct methods using SHELXT2014/5. ${ }^{[15]}$ Refinement was performed against $F^{2}$ on all data by full-matrix least squares using SHELXL2018/3. ${ }^{[16]}$ All nonhydrogen atoms were refined anisotropically and $\mathrm{C}-\mathrm{H}$ hydrogen atoms were positioned at geometrically calculated positions and refined using a riding model. The isotropic displacement parameters of all hydrogen atoms were fixed to $1.2 \mathrm{x}$ the $\mathrm{U}_{\mathrm{eq}}$ value of the atoms they are linked to.

The crystallographic data were deposited at the Cambridge Crystallographic Data Centre as CCDC No. 2098838-2098839 and can be obtained free of charge. ${ }^{[17]}$ 


\section{1. (1Z) Z-2,2'-Diethynyl-9,9'-bifluorenylidene}

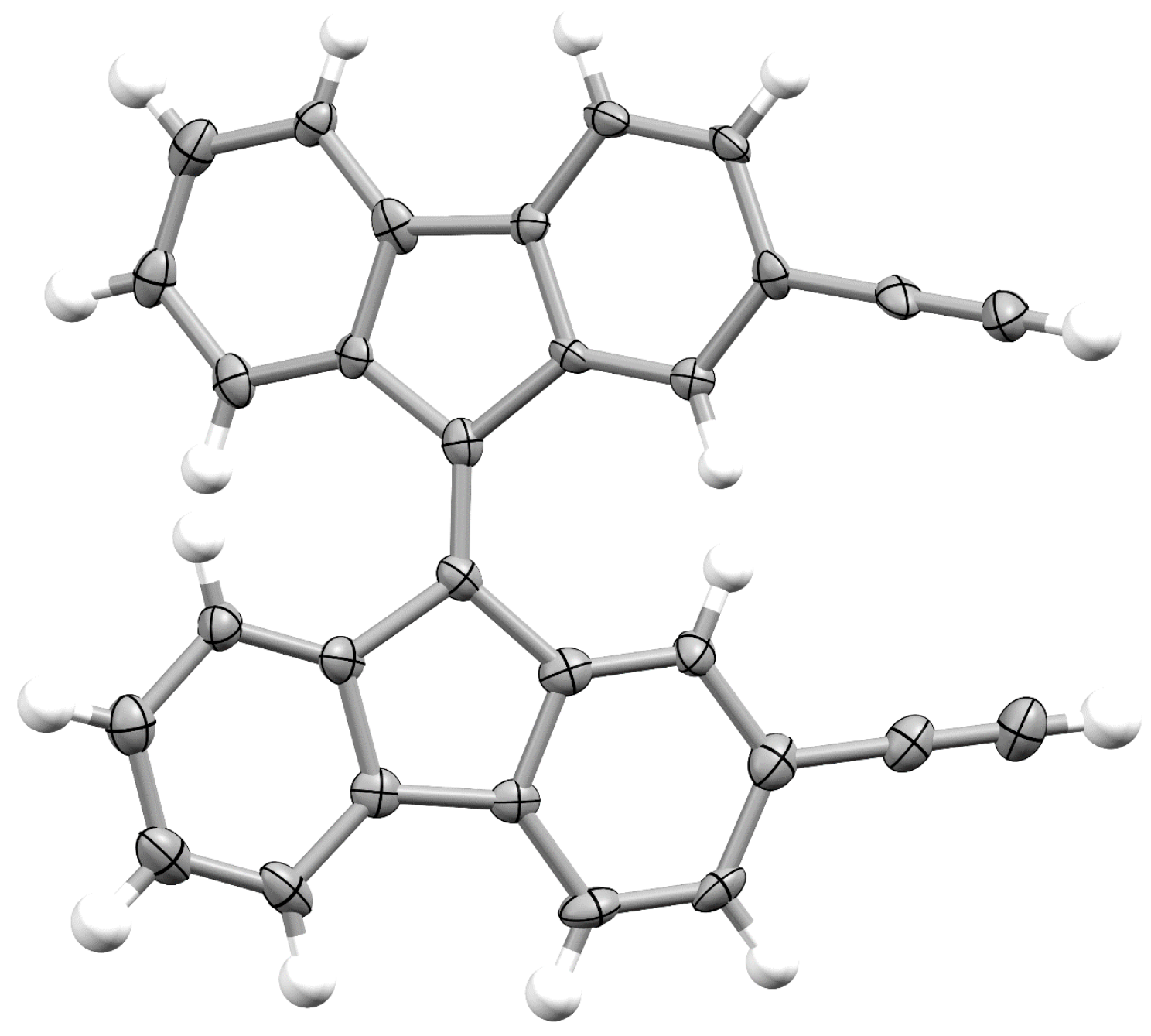

Figure S13. - Thermal ellipsoid plot for $\mathbf{1 Z}$ with $50 \%$ probability level.

The crystal structure was solved in the orthorhombic space group $P c a 2_{1}$ and the asymmetric unit contains two independent molecules of $\mathbf{1 Z}$.

Table S4. Crystal data and structure refinement for $\mathbf{I Z}$.

CCDC No

Empirical formula

Formula weight

Temperature

Wavelength

Crystal system

Space group

Unit cell dimensions

Volume
2098838

$\mathrm{C}_{30} \mathrm{H}_{16}$

376.43

100(2) K

$0.71073 \AA$

Orthorhombic

Pca2 1

$\mathrm{a}=23.113(2) \AA$

$\alpha=90^{\circ}$.

$\mathrm{b}=7.6042(9) \AA$

$\beta=90^{\circ}$.

$\mathrm{c}=21.422(2) \AA$

$\gamma=90^{\circ}$.

3765.1(7) $\AA^{3}$ 
Density (calculated)

Absorption coefficient

$F(000)$

Crystal size

Theta range for data collection

Index ranges

Reflections collected

Independent reflections

Completeness to theta $=25.242^{\circ}$

Absorption correction

Refinement method

Data / restraints / parameters

Goodness-of-fit on $F^{2}$

Final $R$ indices $[I>2 \sigma(I)]$

$\mathrm{R}$ indices (all data)

Absolute structure parameter

Largest diff. peak and hole
$1.328 \mathrm{Mg} / \mathrm{m}^{3}$

$0.075 \mathrm{~mm}^{-1}$

1568

$0.993 \times 0.046 \times 0.044 \mathrm{~mm}^{3}$

1.762 to $27.107^{\circ}$.

$-29 \leq \mathrm{h} \leq 29,-9 \leq \mathrm{k} \leq 9,-27 \leq 1 \leq 27$

66757

$8310[\mathrm{R}(\mathrm{int})=0.1574]$

$100.0 \%$

Semi-empirical from equivalents

Full-matrix least-squares on $F^{2}$

$8310 / 1 / 541$

1.027

$\mathrm{R} 1=0.0930, \mathrm{wR} 2=0.2206$

$\mathrm{R} 1=0.1318, \mathrm{wR} 2=0.2554$

not determined

0.734 and -0.382 e. $\AA^{-3}$ 
Table S5. Atomic coordinates $\left(x 10^{4}\right)$ and equivalent isotropic displacement parameters $\left(\AA^{2} \times 10^{3}\right)$ for 1Z. U(eq) is defined as one third of the trace of the orthogonalized $U^{i j}$ tensor.

\begin{tabular}{|c|c|c|c|c|}
\hline & $\mathrm{x}$ & $\mathrm{y}$ & $\mathrm{z}$ & $\mathrm{U}(\mathrm{eq})$ \\
\hline $\mathrm{C}(1)$ & $4150(2)$ & $676(8)$ & $4200(3)$ & $18(1)$ \\
\hline$C(2)$ & $3591(2)$ & $1389(8)$ & $4231(3)$ & $17(1)$ \\
\hline$C(3)$ & $3187(2)$ & 1619(8) & $4758(3)$ & $21(1)$ \\
\hline $\mathrm{C}(4)$ & $2635(2)$ & 2212(9) & $4471(3)$ & $20(1)$ \\
\hline$C(5)$ & $2125(3)$ & $2854(8)$ & $4730(3)$ & $25(1)$ \\
\hline$C(6)$ & $1686(3)$ & $3453(9)$ & $4341(3)$ & $27(2)$ \\
\hline$C(7)$ & $1751(3)$ & 3461(9) & $3700(3)$ & $31(2)$ \\
\hline $\mathrm{C}(8)$ & $2268(2)$ & 2900(9) & $3438(3)$ & $22(1)$ \\
\hline$C(9)$ & $2707(3)$ & $2284(8)$ & $3825(3)$ & $23(1)$ \\
\hline$C(10)$ & $3296(2)$ & 1761(8) & $3668(3)$ & $18(1)$ \\
\hline $\mathrm{C}(11)$ & $3556(3)$ & $1525(9)$ & $3088(3)$ & $23(1)$ \\
\hline$C(12)$ & $4116(2)$ & $890(9)$ & $3068(3)$ & $22(1)$ \\
\hline$C(13)$ & $4414(2)$ & $448(8)$ & $3623(3)$ & $21(1)$ \\
\hline$C(14)$ & 4994(3) & $-190(10)$ & $3584(3)$ & $24(1)$ \\
\hline$C(15)$ & $5479(3)$ & $-655(10)$ & $3552(3)$ & $32(2)$ \\
\hline $\mathrm{C}(21)$ & $4359(2)$ & $2285(9)$ & $5571(3)$ & $24(1)$ \\
\hline $\mathrm{C}(22)$ & $3824(3)$ & $1600(8)$ & $5744(3)$ & $24(1)$ \\
\hline $\mathrm{C}(23)$ & $3287(2)$ & $1328(8)$ & $5383(3)$ & $20(1)$ \\
\hline$C(24)$ & $2849(3)$ & 743(9) & $5854(3)$ & $25(2)$ \\
\hline$C(25)$ & $2302(2)$ & 21(9) & $5768(3)$ & $25(1)$ \\
\hline$C(26)$ & $2002(3)$ & $-576(10)$ & $6290(3)$ & $31(2)$ \\
\hline $\mathrm{C}(27)$ & $2252(3)$ & $-480(10)$ & $6881(3)$ & $34(2)$ \\
\hline$C(28)$ & 2801(3) & $160(10)$ & $6962(3)$ & $30(2)$ \\
\hline $\mathrm{C}(29)$ & $3109(2)$ & $748(8)$ & $6447(3)$ & $23(1)$ \\
\hline$C(30)$ & $3709(2)$ & 1342(8) & $6389(3)$ & $20(1)$ \\
\hline$C(31)$ & $4121(3)$ & 1666(9) & $6839(3)$ & $29(2)$ \\
\hline$C(32)$ & $4659(3)$ & $2238(9)$ & $6660(3)$ & $26(2)$ \\
\hline$C(33)$ & $4787(3)$ & $2564(10)$ & $6025(3)$ & $29(2)$ \\
\hline$C(34)$ & $5355(3)$ & $3166(9)$ & $5843(3)$ & $28(2)$ \\
\hline$C(35)$ & $5826(3)$ & $3619(10)$ & $5694(4)$ & $36(2)$ \\
\hline$C(41)$ & 7901(3) & $2740(9)$ & $4501(3)$ & $21(1)$ \\
\hline$C(42)$ & $8424(2)$ & $3370(8)$ & $4308(3)$ & $17(1)$ \\
\hline$C(43)$ & $8980(2)$ & $3672(8)$ & $4648(3)$ & $18(1)$ \\
\hline$C(44)$ & $9404(2)$ & $4205(8)$ & $4158(3)$ & $20(1)$ \\
\hline
\end{tabular}




\begin{tabular}{|c|c|c|c|c|}
\hline $\mathrm{C}(45)$ & 9957(3) & 4924(10) & $4215(3)$ & $25(1)$ \\
\hline $\mathrm{C}(46)$ & $10248(3)$ & $5425(9)$ & $3669(3)$ & $29(2)$ \\
\hline $\mathrm{C}(47)$ & 9983(3) & $5219(10)$ & $3084(3)$ & $26(2)$ \\
\hline $\mathrm{C}(48)$ & $9433(3)$ & $4607(9)$ & $3033(3)$ & $26(1)$ \\
\hline $\mathrm{C}(49)$ & $9136(2)$ & $4131(8)$ & $3577(3)$ & $22(1)$ \\
\hline$C(50)$ & $8539(2)$ & $3587(8)$ & $3658(3)$ & $20(1)$ \\
\hline $\mathrm{C}(51)$ & $8107(3)$ & $3260(9)$ & $3218(3)$ & $25(1)$ \\
\hline$C(52)$ & 7574(3) & $2687(9)$ & $3423(3)$ & $21(1)$ \\
\hline$C(53)$ & $7456(3)$ & $2426(8)$ & 4054(3) & $21(1)$ \\
\hline$C(54)$ & 6893(3) & 1901(9) & $4260(3)$ & $25(1)$ \\
\hline$C(55)$ & 6412(3) & $1550(9)$ & $4414(3)$ & $29(2)$ \\
\hline $\mathrm{C}(61)$ & $8144(2)$ & $4419(8)$ & $5848(3)$ & $18(1)$ \\
\hline$C(62)$ & $8693(2)$ & $3659(8)$ & $5806(2)$ & $16(1)$ \\
\hline$C(63)$ & 9091(2) & $3437(8)$ & $5271(3)$ & $17(1)$ \\
\hline$C(64)$ & $9650(3)$ & $2810(9)$ & $5540(3)$ & $20(1)$ \\
\hline$C(65)$ & $10149(2)$ & 2162(9) & $5269(3)$ & $23(1)$ \\
\hline$C(66)$ & 10592(3) & $1529(8)$ & $5648(3)$ & $24(1)$ \\
\hline $\mathrm{C}(67)$ & $10528(2)$ & $1498(9)$ & $6294(3)$ & $25(1)$ \\
\hline $\mathrm{C}(68)$ & 10024(3) & 2061(9) & $6577(3)$ & $22(1)$ \\
\hline $\mathrm{C}(69)$ & $9580(2)$ & 2701(8) & $6195(3)$ & $19(1)$ \\
\hline $\mathrm{C}(70)$ & 8997(2) & $3281(8)$ & 6364(3) & $18(1)$ \\
\hline $\mathrm{C}(71)$ & $8743(3)$ & $3525(9)$ & 6941(3) & $23(1)$ \\
\hline$C(72)$ & 8191(3) & $4206(9)$ & $6975(3)$ & $22(1)$ \\
\hline$C(73)$ & 7879(2) & $4646(8)$ & $6435(3)$ & $17(1)$ \\
\hline$C(74)$ & 7309(3) & $5324(8)$ & $6488(3)$ & $20(1)$ \\
\hline$C(75)$ & $6825(3)$ & $5833(9)$ & $6540(3)$ & $26(1)$ \\
\hline
\end{tabular}


Table S6. Bond lengths $[\AA]$ and angles $\left[{ }^{\circ}\right]$ for $\mathbf{1 Z}$.

\begin{tabular}{|c|c|c|c|}
\hline$C(1)-C(13)$ & $1.391(8)$ & $\mathrm{C}(26)-\mathrm{H}(26)$ & 0.9500 \\
\hline $\mathrm{C}(1)-\mathrm{C}(2)$ & $1.403(8)$ & $\mathrm{C}(27)-\mathrm{C}(28)$ & $1.369(10)$ \\
\hline $\mathrm{C}(1)-\mathrm{H}(1)$ & 0.9500 & $\mathrm{C}(27)-\mathrm{H}(27)$ & 0.9500 \\
\hline$C(2)-C(10)$ & $1.415(8)$ & $\mathrm{C}(28)-\mathrm{C}(29)$ & $1.388(9)$ \\
\hline$C(2)-C(3)$ & $1.474(8)$ & $\mathrm{C}(28)-\mathrm{H}(28)$ & 0.9500 \\
\hline$C(3)-C(23)$ & $1.378(9)$ & $C(29)-C(30)$ & $1.464(8)$ \\
\hline$C(3)-C(4)$ & $1.486(8)$ & $\mathrm{C}(30)-\mathrm{C}(31)$ & $1.379(9)$ \\
\hline$C(4)-C(5)$ & $1.391(9)$ & $C(31)-C(32)$ & $1.372(10)$ \\
\hline$C(4)-C(9)$ & $1.395(9)$ & $\mathrm{C}(31)-\mathrm{H}(31)$ & 0.9500 \\
\hline$C(5)-C(6)$ & $1.390(9)$ & $C(32)-C(33)$ & $1.415(10)$ \\
\hline $\mathrm{C}(5)-\mathrm{H}(5)$ & 0.9500 & $\mathrm{C}(32)-\mathrm{H}(32)$ & 0.9500 \\
\hline$C(6)-C(7)$ & $1.382(10)$ & $\mathrm{C}(33)-\mathrm{C}(34)$ & $1.444(10)$ \\
\hline $\mathrm{C}(6)-\mathrm{H}(6)$ & 0.9500 & $C(34)-C(35)$ & $1.184(10)$ \\
\hline$C(7)-C(8)$ & $1.387(9)$ & $\mathrm{C}(35)-\mathrm{H}(35)$ & 0.9500 \\
\hline $\mathrm{C}(7)-\mathrm{H}(7)$ & 0.9500 & $\mathrm{C}(41)-\mathrm{C}(42)$ & $1.364(8)$ \\
\hline $\mathrm{C}(8)-\mathrm{C}(9)$ & $1.392(9)$ & $\mathrm{C}(41)-\mathrm{C}(53)$ & $1.425(8)$ \\
\hline $\mathrm{C}(8)-\mathrm{H}(8)$ & 0.9500 & $\mathrm{C}(41)-\mathrm{H}(41)$ & 0.9500 \\
\hline$C(9)-C(10)$ & $1.457(9)$ & $C(42)-C(50)$ & $1.425(8)$ \\
\hline$C(10)-C(11)$ & $1.391(8)$ & $C(42)-C(43)$ & $1.496(8)$ \\
\hline$C(11)-C(12)$ & $1.383(9)$ & $C(43)-C(63)$ & $1.370(8)$ \\
\hline $\mathrm{C}(11)-\mathrm{H}(11)$ & 0.9500 & $C(43)-C(44)$ & $1.492(8)$ \\
\hline$C(12)-C(13)$ & $1.414(9)$ & $C(44)-C(49)$ & $1.392(8)$ \\
\hline $\mathrm{C}(12)-\mathrm{H}(12)$ & 0.9500 & $C(44)-C(45)$ & $1.396(8)$ \\
\hline$C(13)-C(14)$ & $1.428(8)$ & $C(45)-C(46)$ & $1.401(8)$ \\
\hline$C(14)-C(15)$ & $1.179(9)$ & $\mathrm{C}(45)-\mathrm{H}(45)$ & 0.9500 \\
\hline $\mathrm{C}(15)-\mathrm{H}(15)$ & 0.9500 & $C(46)-C(47)$ & $1.404(10)$ \\
\hline $\mathrm{C}(21)-\mathrm{C}(22)$ & $1.392(9)$ & $\mathrm{C}(46)-\mathrm{H}(46)$ & 0.9500 \\
\hline $\mathrm{C}(21)-\mathrm{C}(33)$ & $1.403(9)$ & $\mathrm{C}(47)-\mathrm{C}(48)$ & $1.357(9)$ \\
\hline $\mathrm{C}(21)-\mathrm{H}(21)$ & 0.9500 & $\mathrm{C}(47)-\mathrm{H}(47)$ & 0.9500 \\
\hline $\mathrm{C}(22)-\mathrm{C}(30)$ & $1.421(9)$ & $\mathrm{C}(48)-\mathrm{C}(49)$ & $1.402(8)$ \\
\hline $\mathrm{C}(22)-\mathrm{C}(23)$ & $1.476(8)$ & $\mathrm{C}(48)-\mathrm{H}(48)$ & 0.9500 \\
\hline $\mathrm{C}(23)-\mathrm{C}(24)$ & $1.495(8)$ & $C(49)-C(50)$ & $1.449(8)$ \\
\hline $\mathrm{C}(24)-\mathrm{C}(25)$ & $1.390(8)$ & $\mathrm{C}(50)-\mathrm{C}(51)$ & $1.397(8)$ \\
\hline $\mathrm{C}(24)-\mathrm{C}(29)$ & $1.405(9)$ & $C(51)-C(52)$ & $1.379(9)$ \\
\hline$C(25)-C(26)$ & $1.393(9)$ & $\mathrm{C}(51)-\mathrm{H}(51)$ & 0.9500 \\
\hline $\mathrm{C}(25)-\mathrm{H}(25)$ & 0.9500 & $C(52)-C(53)$ & $1.393(9)$ \\
\hline$C(26)-C(27)$ & $1.393(10)$ & $\mathrm{C}(52)-\mathrm{H}(52)$ & 0.9500 \\
\hline
\end{tabular}




\begin{tabular}{|c|c|c|c|}
\hline C(53)-C(54) & $1.430(9)$ & $\mathrm{C}(5)-\mathrm{C}(4)-\mathrm{C}(3)$ & $132.0(6)$ \\
\hline $\mathrm{C}(54)-\mathrm{C}(55)$ & $1.191(9)$ & $\mathrm{C}(9)-\mathrm{C}(4)-\mathrm{C}(3)$ & $108.6(5)$ \\
\hline $\mathrm{C}(55)-\mathrm{H}(55)$ & 0.9500 & $C(6)-C(5)-C(4)$ & $119.6(6)$ \\
\hline $\mathrm{C}(61)-\mathrm{C}(62)$ & $1.398(8)$ & $\mathrm{C}(6)-\mathrm{C}(5)-\mathrm{H}(5)$ & 120.2 \\
\hline $\mathrm{C}(61)-\mathrm{C}(73)$ & $1.410(8)$ & $\mathrm{C}(4)-\mathrm{C}(5)-\mathrm{H}(5)$ & 120.2 \\
\hline $\mathrm{C}(61)-\mathrm{H}(61)$ & 0.9500 & $C(7)-C(6)-C(5)$ & $121.2(6)$ \\
\hline$C(62)-C(70)$ & $1.415(8)$ & $C(7)-C(6)-H(6)$ & 119.4 \\
\hline$C(62)-C(63)$ & $1.480(7)$ & $\mathrm{C}(5)-\mathrm{C}(6)-\mathrm{H}(6)$ & 119.4 \\
\hline $\mathrm{C}(63)-\mathrm{C}(64)$ & $1.492(8)$ & $\mathrm{C}(6)-\mathrm{C}(7)-\mathrm{C}(8)$ & $119.6(6)$ \\
\hline $\mathrm{C}(64)-\mathrm{C}(65)$ & $1.383(9)$ & $\mathrm{C}(6)-\mathrm{C}(7)-\mathrm{H}(7)$ & 120.2 \\
\hline$C(64)-C(69)$ & $1.414(8)$ & $\mathrm{C}(8)-\mathrm{C}(7)-\mathrm{H}(7)$ & 120.2 \\
\hline$C(65)-C(66)$ & $1.392(8)$ & $\mathrm{C}(7)-\mathrm{C}(8)-\mathrm{C}(9)$ & $119.4(6)$ \\
\hline $\mathrm{C}(65)-\mathrm{H}(65)$ & 0.9500 & $\mathrm{C}(7)-\mathrm{C}(8)-\mathrm{H}(8)$ & 120.3 \\
\hline $\mathrm{C}(66)-\mathrm{C}(67)$ & $1.391(9)$ & $\mathrm{C}(9)-\mathrm{C}(8)-\mathrm{H}(8)$ & 120.3 \\
\hline $\mathrm{C}(66)-\mathrm{H}(66)$ & 0.9500 & $\mathrm{C}(8)-\mathrm{C}(9)-\mathrm{C}(4)$ & $121.1(6)$ \\
\hline$C(67)-C(68)$ & $1.383(9)$ & $\mathrm{C}(8)-\mathrm{C}(9)-\mathrm{C}(10)$ & $129.5(6)$ \\
\hline $\mathrm{C}(67)-\mathrm{H}(67)$ & 0.9500 & $C(4)-C(9)-C(10)$ & $109.3(6)$ \\
\hline $\mathrm{C}(68)-\mathrm{C}(69)$ & $1.400(8)$ & $\mathrm{C}(11)-\mathrm{C}(10)-\mathrm{C}(2)$ & $121.9(5)$ \\
\hline $\mathrm{C}(68)-\mathrm{H}(68)$ & 0.9500 & $\mathrm{C}(11)-\mathrm{C}(10)-\mathrm{C}(9)$ & $130.2(6)$ \\
\hline C(69)-C(70) & $1.463(8)$ & $\mathrm{C}(2)-\mathrm{C}(10)-\mathrm{C}(9)$ & $107.9(5)$ \\
\hline$C(70)-C(71)$ & $1.382(8)$ & $C(12)-C(11)-C(10)$ & $118.5(5)$ \\
\hline$C(71)-C(72)$ & $1.379(9)$ & $\mathrm{C}(12)-\mathrm{C}(11)-\mathrm{H}(11)$ & 120.7 \\
\hline $\mathrm{C}(71)-\mathrm{H}(71)$ & 0.9500 & $\mathrm{C}(10)-\mathrm{C}(11)-\mathrm{H}(11)$ & 120.7 \\
\hline$C(72)-C(73)$ & $1.405(9)$ & $\mathrm{C}(11)-\mathrm{C}(12)-\mathrm{C}(13)$ & $120.8(5)$ \\
\hline $\mathrm{C}(72)-\mathrm{H}(72)$ & 0.9500 & $\mathrm{C}(11)-\mathrm{C}(12)-\mathrm{H}(12)$ & 119.6 \\
\hline $\mathrm{C}(73)-\mathrm{C}(74)$ & $1.420(8)$ & $\mathrm{C}(13)-\mathrm{C}(12)-\mathrm{H}(12)$ & 119.6 \\
\hline$C(74)-C(75)$ & $1.188(8)$ & $C(1)-C(13)-C(12)$ & $120.3(5)$ \\
\hline \multirow[t]{2}{*}{$\mathrm{C}(75)-\mathrm{H}(75)$} & 0.9500 & $\mathrm{C}(1)-\mathrm{C}(13)-\mathrm{C}(14)$ & $120.4(5)$ \\
\hline & & $\mathrm{C}(12)-\mathrm{C}(13)-\mathrm{C}(14)$ & $119.3(5)$ \\
\hline $\mathrm{C}(13)-\mathrm{C}(1)-\mathrm{C}(2)$ & $119.7(5)$ & $\mathrm{C}(15)-\mathrm{C}(14)-\mathrm{C}(13)$ & $177.5(8)$ \\
\hline $\mathrm{C}(13)-\mathrm{C}(1)-\mathrm{H}(1)$ & 120.2 & $\mathrm{C}(14)-\mathrm{C}(15)-\mathrm{H}(15)$ & 180.0 \\
\hline $\mathrm{C}(2)-\mathrm{C}(1)-\mathrm{H}(1)$ & 120.2 & $\mathrm{C}(22)-\mathrm{C}(21)-\mathrm{C}(33)$ & $120.0(6)$ \\
\hline$C(1)-C(2)-C(10)$ & $118.7(5)$ & $\mathrm{C}(22)-\mathrm{C}(21)-\mathrm{H}(21)$ & 120.0 \\
\hline $\mathrm{C}(1)-\mathrm{C}(2)-\mathrm{C}(3)$ & $131.7(5)$ & $\mathrm{C}(33)-\mathrm{C}(21)-\mathrm{H}(21)$ & 120.0 \\
\hline$C(10)-C(2)-C(3)$ & $108.9(5)$ & $\mathrm{C}(21)-\mathrm{C}(22)-\mathrm{C}(30)$ & $118.4(6)$ \\
\hline $\mathrm{C}(23)-\mathrm{C}(3)-\mathrm{C}(2)$ & $128.2(5)$ & $C(21)-C(22)-C(23)$ & $131.4(6)$ \\
\hline$C(23)-C(3)-C(4)$ & $126.5(5)$ & $\mathrm{C}(30)-\mathrm{C}(22)-\mathrm{C}(23)$ & $109.4(5)$ \\
\hline$C(2)-C(3)-C(4)$ & $105.3(5)$ & $C(3)-C(23)-C(22)$ & $128.8(5)$ \\
\hline$C(5)-C(4)-C(9)$ & $119.0(6)$ & $\mathrm{C}(3)-\mathrm{C}(23)-\mathrm{C}(24)$ & $126.1(5)$ \\
\hline
\end{tabular}




\begin{tabular}{|c|c|}
\hline $\mathrm{C}(22)-\mathrm{C}(23)-\mathrm{C}(24)$ & $105.0(5)$ \\
\hline $\mathrm{C}(25)-\mathrm{C}(24)-\mathrm{C}(29)$ & $120.5(6)$ \\
\hline $\mathrm{C}(25)-\mathrm{C}(24)-\mathrm{C}(23)$ & $130.1(6)$ \\
\hline $\mathrm{C}(29)-\mathrm{C}(24)-\mathrm{C}(23)$ & $108.7(5)$ \\
\hline $\mathrm{C}(24)-\mathrm{C}(25)-\mathrm{C}(26)$ & $118.5(6)$ \\
\hline $\mathrm{C}(24)-\mathrm{C}(25)-\mathrm{H}(25)$ & 120.8 \\
\hline $\mathrm{C}(26)-\mathrm{C}(25)-\mathrm{H}(25)$ & 120.8 \\
\hline$C(25)-C(26)-C(27)$ & $120.3(6)$ \\
\hline $\mathrm{C}(25)-\mathrm{C}(26)-\mathrm{H}(26)$ & 119.9 \\
\hline $\mathrm{C}(27)-\mathrm{C}(26)-\mathrm{H}(26)$ & 119.9 \\
\hline $\mathrm{C}(28)-\mathrm{C}(27)-\mathrm{C}(26)$ & $121.3(7)$ \\
\hline $\mathrm{C}(28)-\mathrm{C}(27)-\mathrm{H}(27)$ & 119.4 \\
\hline $\mathrm{C}(26)-\mathrm{C}(27)-\mathrm{H}(27)$ & 119.4 \\
\hline C(27)-C(28)-C(29) & $119.2(7)$ \\
\hline $\mathrm{C}(27)-\mathrm{C}(28)-\mathrm{H}(28)$ & 120.4 \\
\hline $\mathrm{C}(29)-\mathrm{C}(28)-\mathrm{H}(28)$ & 120.4 \\
\hline $\mathrm{C}(28)-\mathrm{C}(29)-\mathrm{C}(24)$ & $120.0(6)$ \\
\hline $\mathrm{C}(28)-\mathrm{C}(29)-\mathrm{C}(30)$ & $130.7(6)$ \\
\hline C(24)-C(29)-C(30) & $109.2(5)$ \\
\hline $\mathrm{C}(31)-\mathrm{C}(30)-\mathrm{C}(22)$ & $121.7(6)$ \\
\hline C(31)-C(30)-C(29) & $130.6(6)$ \\
\hline C(22)-C(30)-C(29) & $107.6(5)$ \\
\hline $\mathrm{C}(32)-\mathrm{C}(31)-\mathrm{C}(30)$ & $119.2(6)$ \\
\hline $\mathrm{C}(32)-\mathrm{C}(31)-\mathrm{H}(31)$ & 120.4 \\
\hline $\mathrm{C}(30)-\mathrm{C}(31)-\mathrm{H}(31)$ & 120.4 \\
\hline $\mathrm{C}(31)-\mathrm{C}(32)-\mathrm{C}(33)$ & $120.9(6)$ \\
\hline $\mathrm{C}(31)-\mathrm{C}(32)-\mathrm{H}(32)$ & 119.6 \\
\hline $\mathrm{C}(33)-\mathrm{C}(32)-\mathrm{H}(32)$ & 119.6 \\
\hline $\mathrm{C}(21)-\mathrm{C}(33)-\mathrm{C}(32)$ & $119.5(6)$ \\
\hline $\mathrm{C}(21)-\mathrm{C}(33)-\mathrm{C}(34)$ & $120.2(6)$ \\
\hline $\mathrm{C}(32)-\mathrm{C}(33)-\mathrm{C}(34)$ & $120.3(6)$ \\
\hline $\mathrm{C}(35)-\mathrm{C}(34)-\mathrm{C}(33)$ & $178.4(8)$ \\
\hline $\mathrm{C}(34)-\mathrm{C}(35)-\mathrm{H}(35)$ & 180.0 \\
\hline C(42)-C(41)-C(53) & $119.7(6)$ \\
\hline $\mathrm{C}(42)-\mathrm{C}(41)-\mathrm{H}(41)$ & 120.2 \\
\hline $\mathrm{C}(53)-\mathrm{C}(41)-\mathrm{H}(41)$ & 120.2 \\
\hline$C(41)-C(42)-C(50)$ & $120.1(5)$ \\
\hline $\mathrm{C}(41)-\mathrm{C}(42)-\mathrm{C}(43)$ & $131.9(6)$ \\
\hline $\mathrm{C}(50)-\mathrm{C}(42)-\mathrm{C}(43)$ & $107.3(5)$ \\
\hline
\end{tabular}

\begin{tabular}{|c|c|}
\hline$C(63)-C(43)-C(44)$ & $126.6(5)$ \\
\hline$C(63)-C(43)-C(42)$ & $128.0(5)$ \\
\hline$C(44)-C(43)-C(42)$ & $105.3(5)$ \\
\hline$C(49)-C(44)-C(45)$ & $120.2(5)$ \\
\hline$C(49)-C(44)-C(43)$ & $108.9(5)$ \\
\hline$C(45)-C(44)-C(43)$ & $130.3(6)$ \\
\hline$C(44)-C(45)-C(46)$ & $118.2(6)$ \\
\hline $\mathrm{C}(44)-\mathrm{C}(45)-\mathrm{H}(45)$ & 120.9 \\
\hline $\mathrm{C}(46)-\mathrm{C}(45)-\mathrm{H}(45)$ & 120.9 \\
\hline$C(45)-C(46)-C(47)$ & $120.4(6)$ \\
\hline $\mathrm{C}(45)-\mathrm{C}(46)-\mathrm{H}(46)$ & 119.8 \\
\hline $\mathrm{C}(47)-\mathrm{C}(46)-\mathrm{H}(46)$ & 119.8 \\
\hline$C(48)-C(47)-C(46)$ & $121.2(5)$ \\
\hline $\mathrm{C}(48)-\mathrm{C}(47)-\mathrm{H}(47)$ & 119.4 \\
\hline $\mathrm{C}(46)-\mathrm{C}(47)-\mathrm{H}(47)$ & 119.4 \\
\hline$C(47)-C(48)-C(49)$ & $118.7(6)$ \\
\hline $\mathrm{C}(47)-\mathrm{C}(48)-\mathrm{H}(48)$ & 120.6 \\
\hline $\mathrm{C}(49)-\mathrm{C}(48)-\mathrm{H}(48)$ & 120.6 \\
\hline $\mathrm{C}(44)-\mathrm{C}(49)-\mathrm{C}(48)$ & $121.0(5)$ \\
\hline$C(44)-C(49)-C(50)$ & $109.2(5)$ \\
\hline$C(48)-C(49)-C(50)$ & $129.8(5)$ \\
\hline$C(51)-C(50)-C(42)$ & $120.3(5)$ \\
\hline$C(51)-C(50)-C(49)$ & $130.5(6)$ \\
\hline$C(42)-C(50)-C(49)$ & $109.1(5)$ \\
\hline$C(52)-C(51)-C(50)$ & $118.6(6)$ \\
\hline $\mathrm{C}(52)-\mathrm{C}(51)-\mathrm{H}(51)$ & 120.7 \\
\hline $\mathrm{C}(50)-\mathrm{C}(51)-\mathrm{H}(51)$ & 120.7 \\
\hline$C(51)-C(52)-C(53)$ & $122.0(6)$ \\
\hline $\mathrm{C}(51)-\mathrm{C}(52)-\mathrm{H}(52)$ & 119.0 \\
\hline $\mathrm{C}(53)-\mathrm{C}(52)-\mathrm{H}(52)$ & 119.0 \\
\hline$C(52)-C(53)-C(41)$ & $119.1(6)$ \\
\hline$C(52)-C(53)-C(54)$ & $121.2(6)$ \\
\hline$C(41)-C(53)-C(54)$ & $119.7(6)$ \\
\hline$C(55)-C(54)-C(53)$ & $176.1(7)$ \\
\hline $\mathrm{C}(54)-\mathrm{C}(55)-\mathrm{H}(55)$ & 180.0 \\
\hline$C(62)-C(61)-C(73)$ & $120.1(5)$ \\
\hline $\mathrm{C}(62)-\mathrm{C}(61)-\mathrm{H}(61)$ & 119.9 \\
\hline $\mathrm{C}(73)-\mathrm{C}(61)-\mathrm{H}(61)$ & 119.9 \\
\hline$C(61)-C(62)-C(70)$ & $118.8(5)$ \\
\hline
\end{tabular}




$\begin{array}{llll}\mathrm{C}(61)-\mathrm{C}(62)-\mathrm{C}(63) & 131.4(5) & \mathrm{C}(69)-\mathrm{C}(68)-\mathrm{H}(68) & 121.0 \\ \mathrm{C}(70)-\mathrm{C}(62)-\mathrm{C}(63) & 108.8(5) & \mathrm{C}(68)-\mathrm{C}(69)-\mathrm{C}(64) & 121.2(5) \\ \mathrm{C}(43)-\mathrm{C}(63)-\mathrm{C}(62) & 128.5(5) & \mathrm{C}(68)-\mathrm{C}(69)-\mathrm{C}(70) & 129.4(6) \\ \mathrm{C}(43)-\mathrm{C}(63)-\mathrm{C}(64) & 125.5(5) & \mathrm{C}(64)-\mathrm{C}(69)-\mathrm{C}(70) & 109.5(5) \\ \mathrm{C}(62)-\mathrm{C}(63)-\mathrm{C}(64) & 106.0(5) & \mathrm{C}(71)-\mathrm{C}(70)-\mathrm{C}(62) & 121.1(5) \\ \mathrm{C}(65)-\mathrm{C}(64)-\mathrm{C}(69) & 119.4(6) & \mathrm{C}(71)-\mathrm{C}(70)-\mathrm{C}(69) & 130.8(5) \\ \mathrm{C}(65)-\mathrm{C}(64)-\mathrm{C}(63) & 132.4(5) & \mathrm{C}(62)-\mathrm{C}(70)-\mathrm{C}(69) & 108.0(5) \\ \mathrm{C}(69)-\mathrm{C}(64)-\mathrm{C}(63) & 107.7(5) & \mathrm{C}(72)-\mathrm{C}(71)-\mathrm{C}(70) & 119.4(5) \\ \mathrm{C}(64)-\mathrm{C}(65)-\mathrm{C}(66) & 119.4(6) & \mathrm{C}(72)-\mathrm{C}(71)-\mathrm{H}(71) & 120.3 \\ \mathrm{C}(64)-\mathrm{C}(65)-\mathrm{H}(65) & 120.3 & \mathrm{C}(70)-\mathrm{C}(71)-\mathrm{H}(71) & 120.3 \\ \mathrm{C}(66)-\mathrm{C}(65)-\mathrm{H}(65) & 120.3 & \mathrm{C}(71)-\mathrm{C}(72)-\mathrm{C}(73) & 121.4(5) \\ \mathrm{C}(67)-\mathrm{C}(66)-\mathrm{C}(65) & 120.6(6) & \mathrm{C}(71)-\mathrm{C}(72)-\mathrm{H}(72) & 119.3 \\ \mathrm{C}(67)-\mathrm{C}(66)-\mathrm{H}(66) & 119.7 & \mathrm{C}(73)-\mathrm{C}(72)-\mathrm{H}(72) & 119.3 \\ \mathrm{C}(65)-\mathrm{C}(66)-\mathrm{H}(66) & 119.7 & \mathrm{C}(72)-\mathrm{C}(73)-\mathrm{C}(61) & 118.9(5) \\ \mathrm{C}(68)-\mathrm{C}(67)-\mathrm{C}(66) & 121.3(6) & \mathrm{C}(72)-\mathrm{C}(73)-\mathrm{C}(74) & 119.8(5) \\ \mathrm{C}(68)-\mathrm{C}(67)-\mathrm{H}(67) & 119.4 & \mathrm{C}(61)-\mathrm{C}(73)-\mathrm{C}(74) & 121.3(5) \\ \mathrm{C}(66)-\mathrm{C}(67)-\mathrm{H}(67) & 119.4 & \mathrm{C}(75)-\mathrm{C}(74)-\mathrm{C}(73) & 177.6(7) \\ \mathrm{C}(67)-\mathrm{C}(68)-\mathrm{C}(69) & 118.0(6) & \mathrm{C}(74)-\mathrm{C}(75)-\mathrm{H}(75) & 180.0 \\ \mathrm{C}(67)-\mathrm{C}(68)-\mathrm{H}(68) & 121.0 & & \\ & & & \end{array}$


Table S7. Anisotropic displacement parameters $\left(\AA^{2} \times 10^{3}\right)$ for $\mathbf{1 Z}$. The anisotropic displacement factor exponent takes the form: $-2 p^{2}\left[h^{2} a^{* 2} U^{11}+\ldots+2 h k a^{*} b^{*} U^{12}\right]$

\begin{tabular}{|c|c|c|c|c|c|c|}
\hline & $\mathrm{U}^{11}$ & $\mathrm{U}^{22}$ & $\mathrm{U}^{33}$ & $\mathrm{U}^{23}$ & $\mathrm{U}^{13}$ & $\mathrm{U}^{12}$ \\
\hline $\mathrm{C}(1)$ & 21(3) & $15(3)$ & 19(3) & $-1(2)$ & $0(2)$ & $0(2)$ \\
\hline$C(2)$ & $22(3)$ & $17(3)$ & 13(3) & $1(2)$ & $4(2)$ & $-3(2)$ \\
\hline$C(3)$ & 19(3) & $17(3)$ & 26(3) & $-2(3)$ & $3(2)$ & $0(2)$ \\
\hline$C(4)$ & $17(3)$ & $20(3)$ & $22(3)$ & $0(2)$ & $0(2)$ & $-7(2)$ \\
\hline$C(5)$ & $23(3)$ & $21(4)$ & $30(3)$ & $-2(3)$ & $5(2)$ & $-4(3)$ \\
\hline$C(6)$ & $20(3)$ & $20(4)$ & $42(4)$ & $3(3)$ & $-2(3)$ & $0(3)$ \\
\hline$C(7)$ & $23(3)$ & $23(4)$ & $45(4)$ & $6(3)$ & $-8(3)$ & $-2(3)$ \\
\hline $\mathrm{C}(8)$ & $20(3)$ & $19(4)$ & 26(3) & $2(3)$ & $-3(2)$ & $1(2)$ \\
\hline$C(9)$ & $26(3)$ & 11(3) & $31(3)$ & $2(3)$ & $8(3)$ & $-1(2)$ \\
\hline$C(10)$ & $19(3)$ & $17(3)$ & 19(3) & $0(2)$ & $-2(2)$ & $-6(2)$ \\
\hline $\mathrm{C}(11)$ & $28(3)$ & $27(4)$ & 14(3) & $2(2)$ & $1(2)$ & $-10(3)$ \\
\hline$C(12)$ & $24(3)$ & $28(4)$ & $15(3)$ & $2(2)$ & $6(2)$ & $-7(3)$ \\
\hline$C(13)$ & $20(3)$ & $18(3)$ & $25(3)$ & $4(3)$ & $5(2)$ & $-4(2)$ \\
\hline$C(14)$ & $26(3)$ & $28(4)$ & 17(3) & $-9(3)$ & $3(2)$ & $-3(3)$ \\
\hline$C(15)$ & $25(3)$ & $45(5)$ & 28(3) & $-4(3)$ & 2(3) & $2(3)$ \\
\hline $\mathrm{C}(21)$ & $20(3)$ & $29(4)$ & $22(3)$ & $-6(3)$ & $2(2)$ & $2(3)$ \\
\hline$C(22)$ & $30(3)$ & $17(3)$ & $25(3)$ & $-4(3)$ & $-3(3)$ & $4(3)$ \\
\hline$C(23)$ & $24(3)$ & $16(3)$ & 21(3) & $0(2)$ & $4(2)$ & $3(2)$ \\
\hline$C(24)$ & $23(3)$ & $29(4)$ & 24(3) & $0(3)$ & $3(2)$ & $13(3)$ \\
\hline$C(25)$ & 21(3) & $28(4)$ & $27(3)$ & $-2(3)$ & 1(3) & $2(3)$ \\
\hline$C(26)$ & $28(3)$ & $27(4)$ & $39(4)$ & $0(3)$ & $4(3)$ & $3(3)$ \\
\hline$C(27)$ & $39(4)$ & $29(4)$ & $35(4)$ & $9(3)$ & $12(3)$ & $13(3)$ \\
\hline$C(28)$ & $37(4)$ & $23(4)$ & $30(4)$ & $7(3)$ & $14(3)$ & 11(3) \\
\hline$C(29)$ & $28(3)$ & 13(3) & 26(3) & $-3(2)$ & 1(3) & $7(2)$ \\
\hline$C(30)$ & $27(3)$ & 13(3) & 20(3) & $-2(2)$ & $-1(2)$ & $8(2)$ \\
\hline $\mathrm{C}(31)$ & $39(4)$ & $29(4)$ & 21(3) & $-7(3)$ & $-9(3)$ & $7(3)$ \\
\hline$C(32)$ & $25(3)$ & $25(4)$ & 29(3) & $-5(3)$ & $-13(3)$ & $8(3)$ \\
\hline$C(33)$ & $27(3)$ & $26(4)$ & $34(4)$ & $-9(3)$ & $-2(3)$ & $6(3)$ \\
\hline$C(34)$ & $27(3)$ & $21(4)$ & $36(4)$ & $-6(3)$ & $-6(3)$ & $4(3)$ \\
\hline$C(35)$ & $27(3)$ & $34(4)$ & $47(4)$ & $1(3)$ & $-5(3)$ & $0(3)$ \\
\hline $\mathrm{C}(41)$ & $22(3)$ & $24(4)$ & 18(3) & $-2(3)$ & $-8(2)$ & $-3(3)$ \\
\hline $\mathrm{C}(42)$ & $18(3)$ & $16(3)$ & 18(3) & $0(2)$ & $1(2)$ & $5(2)$ \\
\hline $\mathrm{C}(43)$ & $19(3)$ & $16(3)$ & 21(3) & $-3(2)$ & $1(2)$ & $0(2)$ \\
\hline $\mathrm{C}(44)$ & $18(3)$ & $19(3)$ & 24(3) & $0(3)$ & $1(2)$ & $6(2)$ \\
\hline
\end{tabular}




\begin{tabular}{lllllll}
$\mathrm{C}(45)$ & $23(3)$ & $27(4)$ & $26(3)$ & $5(3)$ & $5(3)$ & $0(3)$ \\
$\mathrm{C}(46)$ & $18(3)$ & $25(4)$ & $44(4)$ & $9(3)$ & $5(3)$ & $0(2)$ \\
$\mathrm{C}(47)$ & $27(3)$ & $28(4)$ & $23(3)$ & $12(3)$ & $12(3)$ & $6(3)$ \\
$\mathrm{C}(48)$ & $31(3)$ & $26(4)$ & $19(3)$ & $2(3)$ & $10(2)$ & $8(3)$ \\
$\mathrm{C}(49)$ & $23(3)$ & $21(3)$ & $20(3)$ & $5(3)$ & $0(2)$ & $6(2)$ \\
$\mathrm{C}(50)$ & $24(3)$ & $16(3)$ & $19(3)$ & $-2(2)$ & $-1(2)$ & $6(2)$ \\
$\mathrm{C}(51)$ & $28(3)$ & $26(4)$ & $20(3)$ & $-1(3)$ & $-2(3)$ & $5(3)$ \\
$\mathrm{C}(52)$ & $27(3)$ & $19(3)$ & $18(3)$ & $-1(2)$ & $-6(2)$ & $6(3)$ \\
$\mathrm{C}(53)$ & $19(3)$ & $15(3)$ & $31(3)$ & $0(3)$ & $-4(3)$ & $4(2)$ \\
$\mathrm{C}(54)$ & $27(3)$ & $20(4)$ & $28(3)$ & $-3(3)$ & $-13(3)$ & $3(3)$ \\
$\mathrm{C}(55)$ & $18(3)$ & $29(4)$ & $41(4)$ & $-1(3)$ & $-6(3)$ & $-3(3)$ \\
$\mathrm{C}(61)$ & $20(3)$ & $18(3)$ & $15(3)$ & $2(2)$ & $-3(2)$ & $-3(2)$ \\
$\mathrm{C}(62)$ & $15(3)$ & $17(3)$ & $15(3)$ & $-2(2)$ & $-1(2)$ & $-4(2)$ \\
$\mathrm{C}(63)$ & $20(3)$ & $13(3)$ & $18(3)$ & $0(2)$ & $4(2)$ & $-1(2)$ \\
$\mathrm{C}(64)$ & $21(3)$ & $20(4)$ & $20(3)$ & $4(3)$ & $0(2)$ & $-6(3)$ \\
$\mathrm{C}(65)$ & $17(3)$ & $26(4)$ & $26(3)$ & $0(3)$ & $1(2)$ & $-2(2)$ \\
$\mathrm{C}(66)$ & $18(3)$ & $20(4)$ & $33(3)$ & $3(3)$ & $0(2)$ & $-2(2)$ \\
$\mathrm{C}(67)$ & $21(3)$ & $27(4)$ & $26(3)$ & $-1(3)$ & $-8(2)$ & $-1(3)$ \\
$\mathrm{C}(68)$ & $23(3)$ & $22(4)$ & $22(3)$ & $2(3)$ & $-4(2)$ & $-5(3)$ \\
$\mathrm{C}(69)$ & $19(3)$ & $15(3)$ & $24(3)$ & $-2(3)$ & $3(2)$ & $-4(2)$ \\
$\mathrm{C}(70)$ & $16(3)$ & $16(3)$ & $20(3)$ & $1(2)$ & $2(2)$ & $-3(2)$ \\
$\mathrm{C}(71)$ & $24(3)$ & $27(4)$ & $19(3)$ & $2(3)$ & $-5(2)$ & $-2(3)$ \\
$\mathrm{C}(72)$ & $25(3)$ & $20(4)$ & $20(3)$ & $-3(2)$ & $4(2)$ & $-6(3)$ \\
$\mathrm{C}(73)$ & $24(3)$ & $13(3)$ & $15(3)$ & $-5(2)$ & $-3(2)$ & $-6(2)$ \\
$\mathrm{C}(74)$ & $26(3)$ & $16(3)$ & $19(3)$ & $0(3)$ & $2(2)$ & $-5(2)$ \\
$\mathrm{C}(75)$ & $24(3)$ & $26(4)$ & $29(3)$ & $-2(3)$ & $3(2)$ & $0(3)$ \\
& & & & & & \\
\hline
\end{tabular}


Table S8. Hydrogen coordinates (x $\left.10^{4}\right)$ and isotropic displacement parameters $\left(\AA^{2} \times 10^{3}\right)$ for $1 Z$.

\begin{tabular}{|c|c|c|c|c|}
\hline & $\mathrm{x}$ & $\mathrm{y}$ & $\mathrm{z}$ & $\mathrm{U}(\mathrm{eq})$ \\
\hline $\mathrm{H}(1)$ & 4347 & 351 & 4572 & 22 \\
\hline $\mathrm{H}(5)$ & 2077 & 2884 & 5170 & 29 \\
\hline$H(6)$ & 1335 & 3865 & 4519 & 33 \\
\hline $\mathrm{H}(7)$ & 1444 & 3848 & 3440 & 37 \\
\hline $\mathrm{H}(8)$ & 2322 & 2936 & 2999 & 26 \\
\hline $\mathrm{H}(11)$ & 3353 & 1794 & 2714 & 27 \\
\hline $\mathrm{H}(12)$ & 4303 & 749 & 2676 & 27 \\
\hline $\mathrm{H}(15)$ & 5871 & -1029 & 3527 & 39 \\
\hline $\mathrm{H}(21)$ & 4434 & 2563 & 5147 & 28 \\
\hline $\mathrm{H}(25)$ & 2137 & -63 & 5363 & 30 \\
\hline $\mathrm{H}(26)$ & 1624 & -1052 & 6244 & 37 \\
\hline $\mathrm{H}(27)$ & 2038 & -865 & 7234 & 41 \\
\hline $\mathrm{H}(28)$ & 2969 & 201 & 7367 & 36 \\
\hline $\mathrm{H}(31)$ & 4034 & 1496 & 7268 & 35 \\
\hline $\mathrm{H}(32)$ & 4950 & 2417 & 6967 & 31 \\
\hline $\mathrm{H}(35)$ & 6203 & 3983 & 5576 & 43 \\
\hline $\mathrm{H}(41)$ & 7834 & 2511 & 4930 & 26 \\
\hline $\mathrm{H}(45)$ & 10132 & 5069 & 4613 & 30 \\
\hline $\mathrm{H}(46)$ & 10626 & 5908 & 3695 & 35 \\
\hline $\mathrm{H}(47)$ & 10192 & 5513 & 2717 & 31 \\
\hline $\mathrm{H}(48)$ & 9254 & 4503 & 2636 & 31 \\
\hline $\mathrm{H}(51)$ & 8179 & 3428 & 2785 & 30 \\
\hline $\mathrm{H}(52)$ & 7279 & 2463 & 3126 & 26 \\
\hline $\mathrm{H}(55)$ & 6028 & 1271 & 4536 & 35 \\
\hline $\mathrm{H}(61)$ & 7949 & 4783 & 5480 & 21 \\
\hline $\mathrm{H}(65)$ & 10190 & 2149 & 4827 & 28 \\
\hline $\mathrm{H}(66)$ & 10940 & 1114 & 5465 & 28 \\
\hline $\mathrm{H}(67)$ & 10838 & 1082 & 6545 & 30 \\
\hline $\mathrm{H}(68)$ & 9979 & 2015 & 7018 & 27 \\
\hline $\mathrm{H}(71)$ & 8947 & 3228 & 7312 & 28 \\
\hline $\mathrm{H}(72)$ & 8018 & 4380 & 7373 & 26 \\
\hline $\mathrm{H}(75)$ & 6438 & 6240 & 6581 & 32 \\
\hline
\end{tabular}




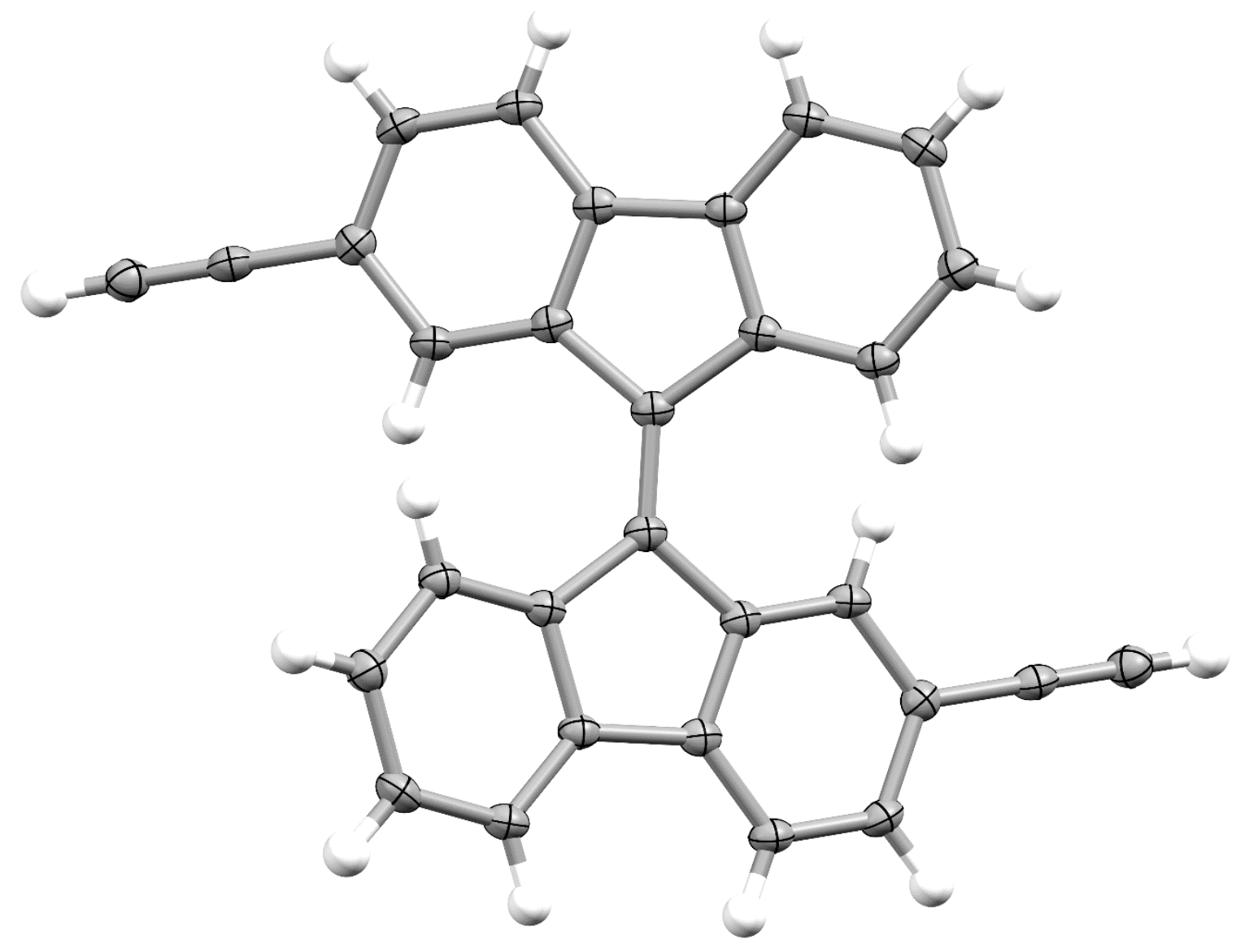

Figure S14. - Thermal ellipsoid plot for $1 \mathbf{Z}$ with 50\% probability level.

$\mathbf{E} \boldsymbol{E}$ was found to crystallize in the monoclinic space group $C 2 / c$. The asymmetric unit contains one half molecule where the second half is generated by two-fold rotation.

Table S9. Crystal data and structure refinement for $\mathbf{1 E}$.

CCDC No

Empirical formula

Formula weight

Temperature

Wavelength

Crystal system

Space group

Unit cell dimensions

Volume

Z

Density (calculated)
2098839

$\mathrm{C}_{30} \mathrm{H}_{16}$

376.43

100(2) K

$0.71073 \AA$

Monoclinic

$C 2 / c$

$\mathrm{a}=14.7137(19) \AA \quad \alpha=90^{\circ}$.

$\mathrm{b}=10.1735(13) \AA \quad \beta=91.364(4)^{\circ}$.

$\mathrm{c}=12.7275(16) \AA \quad \gamma=90^{\circ}$.

1904.6(4) $\AA^{3}$

4

$1.313 \mathrm{Mg} / \mathrm{m}^{3}$ 
Absorption coefficient

$F(000)$

Crystal size

Theta range for data collection

Index ranges

Reflections collected

Independent reflections

Completeness to theta $=25.242^{\circ}$

Absorption correction

Refinement method

Data / restraints / parameters

Goodness-of-fit on $F^{2}$

Final R indices $[\mathrm{I}>2 \sigma(\mathrm{I})]$

$\mathrm{R}$ indices (all data)

Largest diff. peak and hole
$0.075 \mathrm{~mm}^{-1}$

784

$0.090 \times 0.084 \times 0.034 \mathrm{~mm}^{3}$

2.434 to $25.681^{\circ}$.

$-17 \leq \mathrm{h} \leq 17,-12 \leq \mathrm{k} \leq 12,-15 \leq \mathrm{l} \leq 15$

17984

$1804[\mathrm{R}($ int $)=0.0912]$

$100.0 \%$

Semi-empirical from equivalents

Full-matrix least-squares on $F^{2}$

1804 / 0 / 136

1.042

$\mathrm{R} 1=0.0443, \mathrm{wR} 2=0.0989$

$\mathrm{R} 1=0.0727, \mathrm{wR} 2=0.1134$

0.160 and -0.198 e. $\AA^{-3}$ 
Table S10. Atomic coordinates (x $\left.10^{4}\right)$ and equivalent isotropic displacement parameters $\left(\AA^{2} \times 10^{3}\right)$ for $1 E$. U(eq) is defined as one third of the trace of the orthogonalized $U^{i j}$ tensor.

\begin{tabular}{lcccc}
\hline & $\mathrm{x}$ & $\mathrm{y}$ & $\mathrm{z}$ & $\mathrm{U}(\mathrm{eq})$ \\
\hline $\mathrm{C}(1)$ & & & \\
$\mathrm{C}(2)$ & $6856(1)$ & $5472(2)$ & $2586(1)$ & $20(1)$ \\
$\mathrm{C}(3)$ & $6187(1)$ & $5983(2)$ & $3217(1)$ & $18(1)$ \\
$\mathrm{C}(4)$ & $5215(1)$ & $6268(2)$ & $2986(1)$ & $18(1)$ \\
$\mathrm{C}(5)$ & $4826(1)$ & $6610(2)$ & $4016(1)$ & $18(1)$ \\
$\mathrm{C}(6)$ & $3990(1)$ & $7150(2)$ & $4276(1)$ & $20(1)$ \\
$\mathrm{C}(7)$ & $3839(1)$ & $7527(2)$ & $5306(1)$ & $23(1)$ \\
$\mathrm{C}(8)$ & $4522(1)$ & $7398(2)$ & $6083(1)$ & $24(1)$ \\
$\mathrm{C}(9)$ & $5372(1)$ & $6929(2)$ & $5822(1)$ & $21(1)$ \\
$\mathrm{C}(10)$ & $5525(1)$ & $6558(2)$ & $4798(1)$ & $18(1)$ \\
$\mathrm{C}(11)$ & $6365(1)$ & $6145(2)$ & $4300(1)$ & $19(1)$ \\
$\mathrm{C}(12)$ & $7227(1)$ & $5905(2)$ & $4731(1)$ & $21(1)$ \\
$\mathrm{C}(13)$ & $7906(1)$ & $5463(2)$ & $4091(1)$ & $22(1)$ \\
$\mathrm{C}(14)$ & $7727(1)$ & $5222(2)$ & $3020(1)$ & $20(1)$ \\
$\mathrm{C}(15)$ & $8432(1)$ & $4741(2)$ & $2360(1)$ & $22(1)$ \\
\hline & $9020(1)$ & $4362(2)$ & $1810(2)$ & $25(1)$ \\
& & & & \\
\hline
\end{tabular}


Table S11. Bond lengths [Å] and angles $\left[^{\circ}\right]$ for $\mathbf{1 E}$.

\begin{tabular}{|c|c|c|c|}
\hline $\mathrm{C}(1)-\mathrm{C}(2)$ & $1.386(2)$ & $\mathrm{C}(5)-\mathrm{C}(4)-\mathrm{C}(3)$ & $131.57(16)$ \\
\hline$C(1)-C(13)$ & $1.407(2)$ & $C(9)-C(4)-C(3)$ & $108.87(15)$ \\
\hline $\mathrm{C}(1)-\mathrm{H}(1)$ & 0.9500 & $\mathrm{C}(6)-\mathrm{C}(5)-\mathrm{C}(4)$ & $119.68(16)$ \\
\hline$C(2)-C(10)$ & $1.406(2)$ & $\mathrm{C}(6)-\mathrm{C}(5)-\mathrm{H}(5)$ & 120.2 \\
\hline $\mathrm{C}(2)-\mathrm{C}(3)$ & $1.482(2)$ & $\mathrm{C}(4)-\mathrm{C}(5)-\mathrm{H}(5)$ & 120.2 \\
\hline$C(3)-C(3) \# 1$ & $1.375(3)$ & $C(5)-C(6)-C(7)$ & $120.85(18)$ \\
\hline $\mathrm{C}(3)-\mathrm{C}(4)$ & $1.485(2)$ & $\mathrm{C}(5)-\mathrm{C}(6)-\mathrm{H}(6)$ & 119.6 \\
\hline$C(4)-C(5)$ & $1.393(2)$ & $\mathrm{C}(7)-\mathrm{C}(6)-\mathrm{H}(6)$ & 119.6 \\
\hline $\mathrm{C}(4)-\mathrm{C}(9)$ & $1.416(2)$ & $\mathrm{C}(8)-\mathrm{C}(7)-\mathrm{C}(6)$ & $120.02(17)$ \\
\hline$C(5)-C(6)$ & $1.389(3)$ & $\mathrm{C}(8)-\mathrm{C}(7)-\mathrm{H}(7)$ & 120.0 \\
\hline $\mathrm{C}(5)-\mathrm{H}(5)$ & 0.9500 & $\mathrm{C}(6)-\mathrm{C}(7)-\mathrm{H}(7)$ & 120.0 \\
\hline$C(6)-C(7)$ & $1.399(2)$ & $\mathrm{C}(9)-\mathrm{C}(8)-\mathrm{C}(7)$ & $119.19(16)$ \\
\hline $\mathrm{C}(6)-\mathrm{H}(6)$ & 0.9500 & $\mathrm{C}(9)-\mathrm{C}(8)-\mathrm{H}(8)$ & 120.4 \\
\hline$C(7)-C(8)$ & $1.387(3)$ & $\mathrm{C}(7)-\mathrm{C}(8)-\mathrm{H}(8)$ & 120.4 \\
\hline $\mathrm{C}(7)-\mathrm{H}(7)$ & 0.9500 & $\mathrm{C}(8)-\mathrm{C}(9)-\mathrm{C}(4)$ & $121.49(16)$ \\
\hline $\mathrm{C}(8)-\mathrm{C}(9)$ & $1.380(2)$ & $\mathrm{C}(8)-\mathrm{C}(9)-\mathrm{C}(10)$ & $130.22(16)$ \\
\hline $\mathrm{C}(8)-\mathrm{H}(8)$ & 0.9500 & $\mathrm{C}(4)-\mathrm{C}(9)-\mathrm{C}(10)$ & $108.19(15)$ \\
\hline$C(9)-C(10)$ & $1.464(2)$ & $\mathrm{C}(11)-\mathrm{C}(10)-\mathrm{C}(2)$ & $120.85(17)$ \\
\hline$C(10)-C(11)$ & $1.392(2)$ & $\mathrm{C}(11)-\mathrm{C}(10)-\mathrm{C}(9)$ & $130.54(17)$ \\
\hline $\mathrm{C}(11)-\mathrm{C}(12)$ & $1.380(3)$ & $\mathrm{C}(2)-\mathrm{C}(10)-\mathrm{C}(9)$ & $108.61(15)$ \\
\hline $\mathrm{C}(11)-\mathrm{H}(11)$ & 0.9500 & $\mathrm{C}(12)-\mathrm{C}(11)-\mathrm{C}(10)$ & $119.32(17)$ \\
\hline $\mathrm{C}(12)-\mathrm{C}(13)$ & $1.404(3)$ & $\mathrm{C}(12)-\mathrm{C}(11)-\mathrm{H}(11)$ & 120.3 \\
\hline $\mathrm{C}(12)-\mathrm{H}(12)$ & 0.9500 & $\mathrm{C}(10)-\mathrm{C}(11)-\mathrm{H}(11)$ & 120.3 \\
\hline$C(13)-C(14)$ & $1.437(3)$ & $\mathrm{C}(11)-\mathrm{C}(12)-\mathrm{C}(13)$ & $120.62(16)$ \\
\hline$C(14)-C(15)$ & $1.189(3)$ & $\mathrm{C}(11)-\mathrm{C}(12)-\mathrm{H}(12)$ & 119.7 \\
\hline \multirow[t]{2}{*}{$\mathrm{C}(15)-\mathrm{H}(15)$} & 0.9500 & $\mathrm{C}(13)-\mathrm{C}(12)-\mathrm{H}(12)$ & 119.7 \\
\hline & & $C(12)-C(13)-C(1)$ & $119.82(16)$ \\
\hline $\mathrm{C}(2)-\mathrm{C}(1)-\mathrm{C}(13)$ & $119.56(16)$ & $\mathrm{C}(12)-\mathrm{C}(13)-\mathrm{C}(14)$ & $120.39(15)$ \\
\hline $\mathrm{C}(2)-\mathrm{C}(1)-\mathrm{H}(1)$ & 120.2 & $C(1)-C(13)-C(14)$ & $119.78(16)$ \\
\hline $\mathrm{C}(13)-\mathrm{C}(1)-\mathrm{H}(1)$ & 120.2 & $\mathrm{C}(15)-\mathrm{C}(14)-\mathrm{C}(13)$ & $179.0(2)$ \\
\hline$C(1)-C(2)-C(10)$ & $119.59(16)$ & $\mathrm{C}(14)-\mathrm{C}(15)-\mathrm{H}(15)$ & 180.0 \\
\hline $\mathrm{C}(1)-\mathrm{C}(2)-\mathrm{C}(3)$ & $130.75(16)$ & & \\
\hline $\mathrm{C}(10)-\mathrm{C}(2)-\mathrm{C}(3)$ & $109.18(15)$ & & \\
\hline $\mathrm{C}(3) \# 1-\mathrm{C}(3)-\mathrm{C}(2)$ & $126.9(2)$ & & \\
\hline$C(3) \# 1-C(3)-C(4)$ & $128.0(2)$ & & \\
\hline$C(2)-C(3)-C(4)$ & $105.12(14)$ & & \\
\hline $\mathrm{C}(5)-\mathrm{C}(4)-\mathrm{C}(9)$ & $118.54(16)$ & & \\
\hline
\end{tabular}


Symmetry transformations used to generate equivalent atoms:

$\# 1-\mathrm{x}+1, \mathrm{y},-\mathrm{z}+1 / 2$ 
Table S12. Anisotropic displacement parameters $\left(\AA^{2} \times 10^{3}\right)$ for $\mathbf{1 E}$. The anisotropic displacement factor exponent takes the form: $-2 p^{2}\left[h^{2} a^{* 2} U^{11}+\ldots+2 h k a^{*} b^{*} U^{12}\right]$

\begin{tabular}{lllllll}
\hline & $\mathrm{U}^{11}$ & $\mathrm{U}^{22}$ & $\mathrm{U}^{33}$ & $\mathrm{U}^{23}$ & $\mathrm{U}^{13}$ & $\mathrm{U}^{12}$ \\
\hline $\mathrm{C}(1)$ & $23(1)$ & $19(1)$ & $17(1)$ & $1(1)$ & $-4(1)$ & $0(1)$ \\
$\mathrm{C}(2)$ & $20(1)$ & $14(1)$ & $19(1)$ & $2(1)$ & $-2(1)$ & $-1(1)$ \\
$\mathrm{C}(3)$ & $21(1)$ & $15(1)$ & $19(1)$ & $1(1)$ & $-3(1)$ & $0(1)$ \\
$\mathrm{C}(4)$ & $22(1)$ & $16(1)$ & $17(1)$ & $1(1)$ & $-2(1)$ & $-2(1)$ \\
$\mathrm{C}(5)$ & $22(1)$ & $18(1)$ & $20(1)$ & $2(1)$ & $-2(1)$ & $-1(1)$ \\
$\mathrm{C}(6)$ & $24(1)$ & $22(1)$ & $23(1)$ & $1(1)$ & $1(1)$ & $0(1)$ \\
$\mathrm{C}(7)$ & $31(1)$ & $24(1)$ & $17(1)$ & $-1(1)$ & $2(1)$ & $0(1)$ \\
$\mathrm{C}(8)$ & $26(1)$ & $21(1)$ & $17(1)$ & $1(1)$ & $-3(1)$ & $0(1)$ \\
$\mathrm{C}(9)$ & $21(1)$ & $16(1)$ & $18(1)$ & $1(1)$ & $-2(1)$ & $-1(1)$ \\
$\mathrm{C}(10)$ & $23(1)$ & $13(1)$ & $20(1)$ & $2(1)$ & $-3(1)$ & $-2(1)$ \\
$\mathrm{C}(11)$ & $23(1)$ & $20(1)$ & $19(1)$ & $2(1)$ & $-5(1)$ & $0(1)$ \\
$\mathrm{C}(12)$ & $19(1)$ & $21(1)$ & $25(1)$ & $2(1)$ & $-6(1)$ & $0(1)$ \\
$\mathrm{C}(13)$ & $20(1)$ & $17(1)$ & $22(1)$ & $1(1)$ & $0(1)$ & $-1(1)$ \\
$\mathrm{C}(14)$ & $23(1)$ & $21(1)$ & $22(1)$ & $2(1)$ & $-7(1)$ & $0(1)$ \\
$\mathrm{C}(15)$ & $25(1)$ & $25(1)$ & $25(1)$ & $0(1)$ & $-2(1)$ & $2(1)$ \\
\hline & & & & & \\
\hline
\end{tabular}


Table S13. Hydrogen coordinates $\left(x 10^{4}\right)$ and isotropic displacement parameters $\left(A^{2} \times 10^{3}\right)$ for $1 E$.

\begin{tabular}{|c|c|c|c|c|}
\hline & $\mathrm{x}$ & $\mathrm{y}$ & z & $\mathrm{U}(\mathrm{eq})$ \\
\hline $\mathrm{H}(1)$ & 6727 & 5292 & 1866 & 24 \\
\hline $\mathrm{H}(5)$ & 3526 & 7258 & 3751 & 24 \\
\hline $\mathrm{H}(6)$ & 3264 & 7877 & 5485 & 28 \\
\hline $\mathrm{H}(7)$ & 4403 & 7633 & 6788 & 29 \\
\hline $\mathrm{H}(8)$ & 5844 & 6862 & 6342 & 25 \\
\hline $\mathrm{H}(11)$ & 7347 & 6045 & 5459 & 25 \\
\hline $\mathrm{H}(12)$ & 8500 & 5319 & 4377 & 26 \\
\hline $\mathrm{H}(15)$ & 9490 & 4060 & 1372 & 30 \\
\hline
\end{tabular}




\section{Computational Data}

\subsection{DFT-Geometries}

For all optimized geometries no imaginary frequencies were found.

Table S14. B3LYP-D3/def2TZVP optimized geometry of $1 \boldsymbol{E}$.

\begin{tabular}{|c|c|c|c|}
\hline 6 & 0.154211 & 0.667402 & -0.255673 \\
\hline 6 & -0.154211 & -0.667402 & -0.255673 \\
\hline 6 & -0.745621 & 1.790262 & 0.077918 \\
\hline 6 & -0.024564 & 3.002849 & -0.031484 \\
\hline 6 & 1.326423 & 2.694594 & -0.482295 \\
\hline 6 & 1.45058 & 1.28873 & -0.588877 \\
\hline 6 & 0.745621 & -1.790262 & 0.077918 \\
\hline 6 & 0.024564 & -3.002849 & -0.031484 \\
\hline 6 & -1.326423 & -2.694594 & -0.482295 \\
\hline 6 & -1.45058 & -1.28873 & -0.588877 \\
\hline 6 & -2.019497 & 1.803163 & 0.628152 \\
\hline 6 & -2.612833 & 3.022652 & 0.98265 \\
\hline 6 & -1.903329 & 4.222805 & 0.810751 \\
\hline 6 & -0.605551 & 4.211871 & 0.322783 \\
\hline 6 & 2.369956 & 3.536579 & -0.837194 \\
\hline 6 & 3.546648 & 2.979969 & -1.326468 \\
\hline 6 & 3.657515 & 1.59993 & -1.490039 \\
\hline 6 & 2.612833 & 0.752541 & -1.13877 \\
\hline 6 & 2.019497 & -1.803163 & 0.628152 \\
\hline 6 & 2.612833 & -3.022652 & 0.98265 \\
\hline 6 & 1.903329 & -4.222805 & 0.810751 \\
\hline 6 & 0.605551 & -4.211871 & 0.322783 \\
\hline 6 & -2.369956 & -3.536579 & -0.837194 \\
\hline 6 & -3.546648 & -2.979969 & -1.326468 \\
\hline 6 & -3.657515 & -1.59993 & -1.490039 \\
\hline 6 & -2.612833 & -0.752541 & -1.13877 \\
\hline 1 & -2.566125 & 0.890681 & 0.806056 \\
\hline 1 & -2.376259 & 5.154462 & 1.088792 \\
\hline 1 & -0.050739 & 5.137446 & 0.239199 \\
\hline 1 & 2.265371 & 4.610889 & -0.754685 \\
\hline 1 & 4.372053 & 3.622058 & -1.604909 \\
\hline 1 & 2.709979 & -0.308639 & -1.309489 \\
\hline 1 & 2.566125 & -0.890681 & 0.806056 \\
\hline 1 & 2.376259 & -5.154462 & 1.088792 \\
\hline 1 & 0.050739 & -5.137446 & 0.239199 \\
\hline 1 & -2.265371 & -4.610889 & -0.754685 \\
\hline 1 & -4.372053 & -3.622058 & -1.604909 \\
\hline 1 & -4.565092 & -1.18022 & -1.903536 \\
\hline 1 & -2.709979 & 0.308639 & -1.309489 \\
\hline 6 & 3.929466 & -3.040171 & 1.524105 \\
\hline 6 & 5.042252 & -3.053172 & 1.980049 \\
\hline
\end{tabular}




\begin{tabular}{|crrr|}
\hline $\mathbf{1}$ & 6.023896 & -3.063897 & 2.384922 \\
$\mathbf{1}$ & 4.565092 & 1.18022 & -1.903536 \\
$\mathbf{6}$ & -3.929466 & 3.040171 & 1.524105 \\
$\mathbf{6}$ & -5.042252 & 3.053172 & 1.980049 \\
$\mathbf{1}$ & -6.023896 & 3.063897 & 2.384922 \\
\hline $\mathbf{E}$ & $=$ & -1153.24066802 & hartree \\
\hline Grimme-D3 & $=$ & -0.1240836199 & hartree \\
ZPVE & $=$ & 0.351294 & hartree \\
Total Dipole & & & debye \\
moment & & &
\end{tabular}

Table S15. B3LYP-D3/def2TZVP optimized geometry of $1 Z$.

\begin{tabular}{|c|c|c|c|}
\hline 6 & -0.043454 & 0.683589 & -0.646171 \\
\hline 6 & 0.043454 & -0.683589 & -0.646171 \\
\hline 6 & -0.433362 & 1.555757 & -1.770521 \\
\hline 6 & -0.41126 & 2.902458 & -1.336031 \\
\hline 6 & 0.040406 & 2.929816 & 0.049182 \\
\hline 6 & 0.234013 & 1.596416 & 0.481714 \\
\hline 6 & -0.234013 & -1.596416 & 0.481714 \\
\hline 6 & -0.040406 & -2.929816 & 0.049182 \\
\hline 6 & 0.41126 & -2.902458 & -1.336031 \\
\hline 6 & 0.433362 & -1.555757 & -1.770521 \\
\hline 6 & -0.969107 & 1.257119 & -3.021198 \\
\hline 6 & -1.389947 & 2.291407 & -3.849572 \\
\hline 6 & -1.308808 & 3.619952 & -3.434527 \\
\hline 6 & -0.834374 & 3.931071 & -2.164901 \\
\hline 6 & 0.32867 & 3.99913 & 0.884606 \\
\hline 6 & 0.834374 & 3.751852 & 2.151723 \\
\hline 6 & 1.089059 & 2.43729 & 2.576151 \\
\hline 6 & 0.800097 & 1.360369 & 1.726698 \\
\hline 6 & -0.800097 & -1.360369 & 1.726698 \\
\hline 6 & -1.089059 & -2.43729 & 2.576151 \\
\hline 6 & -0.834374 & -3.751852 & 2.151723 \\
\hline 6 & -0.32867 & -3.99913 & 0.884606 \\
\hline 6 & 0.834374 & -3.931071 & -2.164901 \\
\hline 6 & 1.308808 & -3.619952 & -3.434527 \\
\hline 6 & 1.389947 & -2.291407 & -3.849572 \\
\hline 6 & 0.969107 & -1.257119 & -3.021198 \\
\hline 1 & -1.077003 & 0.234745 & -3.349709 \\
\hline 1 & -1.795186 & 2.058952 & -4.825685 \\
\hline 1 & -1.64097 & 4.410403 & -4.094824 \\
\hline 1 & -0.81665 & 4.958247 & -1.823628 \\
\hline 1 & 0.18023 & 5.017779 & 0.550174 \\
\hline
\end{tabular}




\begin{tabular}{|crrr|}
\hline 1 & 1.062182 & 4.570572 & 2.8201 \\
\hline $\mathbf{1}$ & 1.039469 & 0.362456 & 2.058388 \\
\hline $\mathbf{1}$ & -1.039469 & -0.362456 & 2.058388 \\
$\mathbf{1}$ & -1.062182 & -4.570572 & 2.8201 \\
\hline $\mathbf{1}$ & -0.18023 & -5.017779 & 0.550174 \\
$\mathbf{1}$ & 0.81665 & -4.958247 & -1.823628 \\
\hline $\mathbf{1}$ & 1.64097 & -4.410403 & -4.094824 \\
\hline $\mathbf{1}$ & 1.795186 & -2.058952 & -4.825685 \\
$\mathbf{6}$ & 1.077003 & -0.234745 & -3.349709 \\
$\mathbf{6}$ & 1.648364 & 2.195545 & 3.862755 \\
$\mathbf{1}$ & 2.119622 & 1.988266 & 4.949463 \\
\hline $\mathbf{6}$ & 2.534625 & 1.801866 & 5.908962 \\
$\mathbf{6}$ & -1.648364 & -2.195545 & 3.862755 \\
\hline $\mathbf{1}$ & -2.119622 & -1.988266 & 4.949463 \\
\hline $\mathbf{E}$ & -2.534625 & -1.801866 & 5.908962 \\
\hline Grimme-D3 & & & \\
\hline ZPVE & $=$ & -1153.24042345 & hartree \\
\hline Total Dipole moment & $=$ & 0.1241702016 & hartree \\
\hline
\end{tabular}

\subsection{Symmetry Adapted Perturbation Theory (SAPT) of the Dimers}

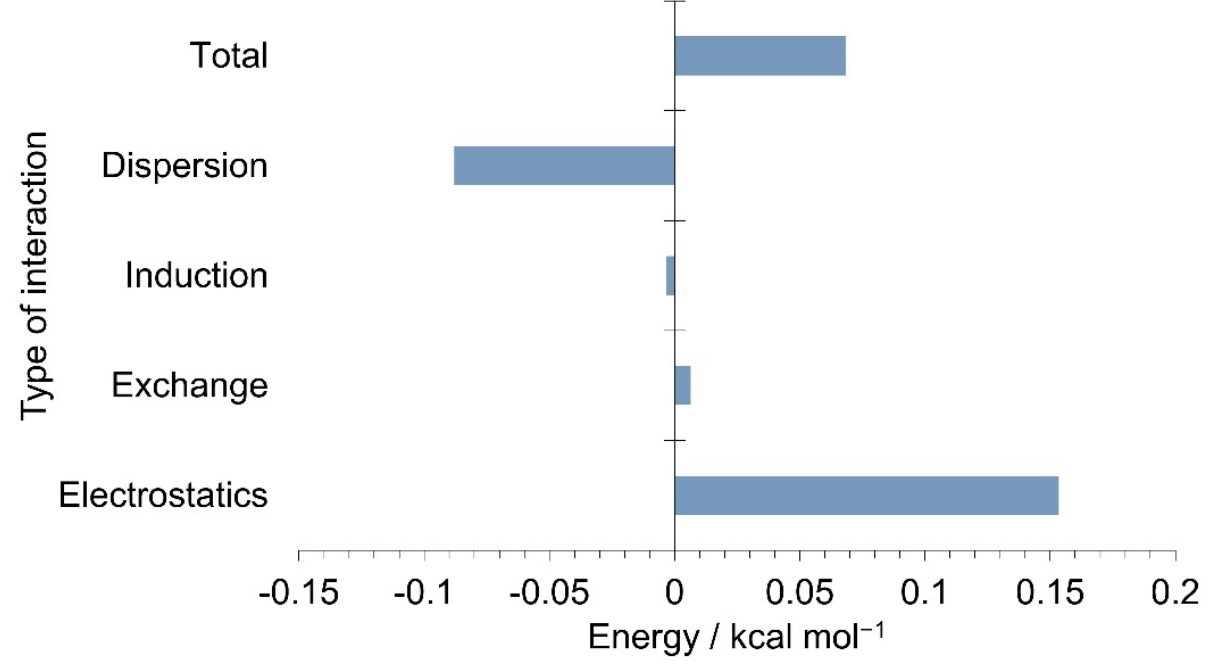

Figure S15. SAPT0/def2TZVP energy decomposition of acetylene dimers based on the optimized geometry of $\mathbf{1 Z}$ after removing the 9,9'-bifluorenylidene moiety and saturating the resulting radicals with hydrogen. 


\subsection{SMD Solvent Model Computations}

For all SMD computations we used "Radii = UAKS" and "Surface = SAS" as additional inputs. The obtained solvent accessible surfaces are listed in figure S15.

Table S16. Solvent accessible surfaces of 1E/Z obtained from B3LYP-D3/def2SVP computations.

$\begin{array}{llllll}\text { Solvent } & r_{\text {solv }} / \AA & \begin{array}{l}\text { SAS (E) } \\ \AA^{2}\end{array} & \begin{array}{l}\text { SAS (Z) } \\ \AA^{2}\end{array} & \Delta S A S(E / Z) / \AA^{2} & \frac{\Delta S A S(E / Z)}{S A S(E)} \\ \%\end{array}$

\subsubsection{E-2,2'-Diethynyl-9,9'-bifluorenylidene}

Methanol:

Table S17. B3LYP-D3/def2SVP (SCRF=SMD, Solvent=Methanol) optimized geometry of $1 \boldsymbol{E}$.

\begin{tabular}{|cccc|}
\hline $\mathbf{6}$ & -0.531453 & -0.440043 & -0.274618 \\
$\mathbf{6}$ & 0.531453 & 0.440043 & -0.274618 \\
$\mathbf{6}$ & -0.497709 & -1.881761 & 0.065963 \\
$\mathbf{6}$ & -1.814123 & -2.410438 & -0.040475 \\
$\mathbf{6}$ & -2.703639 & -1.342426 & -0.495013 \\
$\mathbf{6}$ & -1.942665 & -0.145677 & -0.609793 \\
$\mathbf{6}$ & 0.497709 & 1.881761 & 0.065963 \\
$\mathbf{6}$ & 1.814123 & 2.410438 & -0.040475 \\
$\mathbf{6}$ & 2.703639 & 1.342426 & -0.495013 \\
$\mathbf{6}$ & 1.942665 & 0.145677 & -0.609793 \\
$\mathbf{6}$ & 0.51435 & -2.671797 & 0.612313 \\
$\mathbf{6}$ & 0.246181 & -4.009317 & 0.969478 \\
$\mathbf{6}$ & -1.054679 & -4.533332 & 0.800198 \\
$\mathbf{6}$ & -2.085916 & -3.731827 & 0.313819 \\
$\mathbf{6}$ & -4.053887 & -1.375175 & -0.841887 \\
$\mathbf{6}$ & -4.654663 & -0.212244 & -1.332485 \\
$\mathbf{6}$ & -3.8999 & 0.955455 & -1.504351 \\
$\mathbf{6}$ & -2.545907 & 0.992707 & -1.159895 \\
$\mathbf{6}$ & -0.51435 & 2.671797 & 0.612313 \\
$\mathbf{6}$ & -0.246181 & 4.009317 & 0.969478 \\
$\mathbf{6}$ & 1.054679 & 4.533332 & 0.800198 \\
$\mathbf{6}$ & 2.085916 & 3.731827 & 0.313819 \\
$\mathbf{6}$ & 4.053887 & 1.375175 & -0.841887 \\
$\mathbf{6}$ & 4.654663 & 0.212244 & -1.332485 \\
$\mathbf{6}$ & 3.8999 & -0.955455 & -1.504351 \\
\hline & & & \\
\hline
\end{tabular}




\begin{tabular}{|cccc|}
\hline $\mathbf{6}$ & 2.545907 & -0.992707 & -1.159895 \\
\hline $\mathbf{1}$ & 1.512553 & -2.275488 & 0.787969 \\
\hline $\mathbf{1}$ & -1.247474 & -5.570856 & 1.078614 \\
\hline $\mathbf{1}$ & -3.097717 & -4.134761 & 0.229964 \\
\hline $\mathbf{1}$ & -4.630493 & -2.29865 & -0.751148 \\
\hline $\mathbf{1}$ & -1.712397 & -0.220358 & -1.605988 \\
\hline $\mathbf{1}$ & -1.512553 & 2.275488 & 0.787969 \\
\hline $\mathbf{1}$ & 1.247474 & 5.570856 & 1.078614 \\
\hline $\mathbf{1}$ & 3.097717 & 4.134761 & 0.229964 \\
\hline $\mathbf{1}$ & 4.630493 & 2.29865 & -0.751148 \\
\hline $\mathbf{1}$ & 5.712397 & 0.220358 & -1.605988 \\
\hline $\mathbf{1}$ & 4.371344 & -1.848523 & -1.921053 \\
\hline $\mathbf{1}$ & 1.972698 & -1.901173 & -1.34018 \\
\hline $\mathbf{6}$ & -1.289007 & 4.826186 & 1.508115 \\
\hline $\mathbf{6}$ & -2.17509 & 5.519326 & 1.963709 \\
\hline $\mathbf{1}$ & -2.959168 & 6.132321 & 2.367392 \\
\hline $\mathbf{1}$ & -4.371344 & 1.848523 & -1.921053 \\
\hline $\mathbf{6}$ & 1.289007 & -4.826186 & 1.508115 \\
\hline $\mathbf{6}$ & 2.17509 & -5.519326 & 1.963709 \\
\hline $\mathbf{1}$ & 2.959168 & -6.132321 & 2.367392 \\
\hline & & & $\AA$ \\
\hline SAS & $=$ & 728.9 & Debye \\
\hline Total dipole moment & $=$ & 0.84 & \\
\hline & & & -1.34018 \\
\hline
\end{tabular}

Ethanol:

Table S18. B3LYP-D3/def2SVP (SCRF=SMD, Solvent=Ethanol) optimized geometry of $\mathbf{1 E}$.

\begin{tabular}{|cccc|}
\hline 6 & -0.531465 & -0.440041 & -0.273481 \\
\hline 6 & 0.531465 & 0.440041 & -0.273481 \\
6 & -0.497758 & -1.881869 & 0.066647 \\
6 & -1.814019 & -2.410694 & -0.040665 \\
6 & -2.703493 & -1.342588 & -0.495035 \\
\hline 6 & -1.942661 & -0.145671 & -0.60897 \\
\hline 6 & 0.497758 & 1.881869 & 0.066647 \\
\hline 6 & 1.814019 & 2.410694 & -0.040665 \\
6 & 2.703493 & 1.342588 & -0.495035 \\
\hline 6 & 1.942661 & 0.145671 & -0.60897 \\
6 & 0.514167 & -2.672007 & 0.613119 \\
6 & 0.246165 & -4.009829 & 0.969251 \\
6 & -1.054519 & -4.533969 & 0.799104 \\
6 & -2.085663 & -3.732323 & 0.312816 \\
6 & -4.053612 & -1.375289 & -0.842393 \\
6 & -4.65446 & -0.212232 & -1.332539 \\
6 & -3.899823 & 0.955652 & -1.503488 \\
6 & -2.545939 & 0.992881 & -1.158639 \\
\hline
\end{tabular}




\begin{tabular}{|c|c|c|c|}
\hline 6 & -0.514167 & 2.672007 & 0.613119 \\
\hline 6 & -0.246165 & 4.009829 & 0.969251 \\
\hline 6 & 1.054519 & 4.533969 & 0.799104 \\
\hline 6 & 2.085663 & 3.732323 & 0.312816 \\
\hline 6 & 4.053612 & 1.375289 & -0.842393 \\
\hline 6 & 4.65446 & 0.212232 & -1.332539 \\
\hline 6 & 3.899823 & -0.955652 & -1.503488 \\
\hline 6 & 2.545939 & -0.992881 & -1.158639 \\
\hline 1 & 1.512131 & -2.275546 & 0.789671 \\
\hline 1 & -1.247051 & -5.571702 & 1.076851 \\
\hline 1 & -3.097397 & -4.135264 & 0.228318 \\
\hline 1 & -4.630009 & -2.298943 & -0.752294 \\
\hline 1 & -5.712112 & -0.220349 & -1.606335 \\
\hline 1 & -1.972825 & 1.901531 & -1.338107 \\
\hline 1 & -1.512131 & 2.275546 & 0.789671 \\
\hline 1 & 1.247051 & 5.571702 & 1.076851 \\
\hline 1 & 3.097397 & 4.135264 & 0.228318 \\
\hline 1 & 4.630009 & 2.298943 & -0.752294 \\
\hline 1 & 5.712112 & 0.220349 & -1.606335 \\
\hline 1 & 4.371231 & -1.848963 & -1.919673 \\
\hline 1 & 1.972825 & -1.901531 & -1.338107 \\
\hline 6 & -1.288936 & 4.826958 & 1.507586 \\
\hline 6 & -2.174963 & 5.520406 & 1.962747 \\
\hline 1 & -2.958954 & 6.133525 & 2.366156 \\
\hline 1 & -4.371231 & 1.848963 & -1.919673 \\
\hline 6 & 1.288936 & -4.826958 & 1.507586 \\
\hline 6 & 2.174963 & -5.520406 & 1.962747 \\
\hline 1 & 2.958954 & -6.133525 & 2.366156 \\
\hline SAS & $=$ & 796.1 & $\AA$ \\
\hline Total dipole moment & $=$ & 0.82 & Debye \\
\hline
\end{tabular}

THF:

Table S19. B3LYP-D3/def2SVP (SCRF=SMD, Solvent=TetraHydroFuran) optimized geometry of $1 \boldsymbol{E}$.

\begin{tabular}{cccc}
6 & -0.531529 & -0.440087 & -0.272 \\
\hline 6 & 0.531529 & 0.440087 & -0.272 \\
6 & -0.497946 & -1.882295 & 0.067387 \\
6 & -1.814052 & -2.411272 & -0.040961 \\
6 & -2.703519 & -1.343028 & -0.495084 \\
6 & -1.942961 & -0.14579 & -0.60776 \\
6 & 0.497946 & 1.882295 & 0.067387 \\
\hline 6 & 1.814052 & 2.411272 & -0.040961 \\
6 & 2.703519 & 1.343028 & -0.495084 \\
6 & 1.942961 & 0.14579 & -0.60776 \\
6 & 0.513744 & -2.672932 & 0.613737 \\
\hline
\end{tabular}




\begin{tabular}{|c|c|c|c|}
\hline 6 & 0.245875 & -4.011184 & 0.968667 \\
\hline 6 & -1.054796 & -4.535291 & 0.797631 \\
\hline 6 & -2.085738 & -3.733234 & 0.311472 \\
\hline 6 & -4.053539 & -1.375953 & -0.843065 \\
\hline 6 & -4.654807 & -0.212778 & -1.332548 \\
\hline 6 & -3.900528 & 0.955627 & -1.501984 \\
\hline 6 & -2.546733 & 0.993004 & -1.156504 \\
\hline 6 & -0.513744 & 2.672932 & 0.613737 \\
\hline 6 & -0.245875 & 4.011184 & 0.968667 \\
\hline 6 & 1.054796 & 4.535291 & 0.797631 \\
\hline 6 & 2.085738 & 3.733234 & 0.311472 \\
\hline 6 & 4.053539 & 1.375953 & -0.843065 \\
\hline 6 & 4.654807 & 0.212778 & -1.332548 \\
\hline 6 & 3.900528 & -0.955627 & -1.501984 \\
\hline 6 & 2.546733 & -0.993004 & -1.156504 \\
\hline 1 & 1.511529 & -2.276498 & 0.790561 \\
\hline 1 & -1.247124 & -5.573144 & 1.074606 \\
\hline 1 & -3.097504 & -4.13568 & 0.226075 \\
\hline 1 & -4.629108 & -2.300095 & -0.753691 \\
\hline 1 & -5.71225 & -0.221082 & -1.606749 \\
\hline 1 & -1.973998 & 1.902113 & -1.333971 \\
\hline 1 & -1.511529 & 2.276498 & 0.790561 \\
\hline 1 & 1.247124 & 5.573144 & 1.074606 \\
\hline 1 & 3.097504 & 4.13568 & 0.226075 \\
\hline 1 & 4.629108 & 2.300095 & -0.753691 \\
\hline 1 & 5.71225 & 0.221082 & -1.606749 \\
\hline 1 & 4.371979 & -1.849225 & -1.91721 \\
\hline 1 & 1.973998 & -1.902113 & -1.333971 \\
\hline 6 & -1.288693 & 4.828849 & 1.506529 \\
\hline 6 & -2.174665 & 5.522791 & 1.961149 \\
\hline 1 & -2.958455 & 6.135983 & 2.36436 \\
\hline 1 & -4.371979 & 1.849225 & -1.91721 \\
\hline 6 & 1.288693 & -4.828849 & 1.506529 \\
\hline 6 & 2.174665 & -5.522791 & 1.961149 \\
\hline 1 & 2.958455 & -6.135983 & 2.36436 \\
\hline SAS & $=$ & 875.8 & $\AA$ \\
\hline Total dipole moment & $=$ & 0.79 & Debye \\
\hline
\end{tabular}

Benzene:

Table S20. B3LYP-D3/def2SVP (SCRF=SMD, Solvent=Benzene) optimized geometry of $\mathbf{1 E}$.

$\begin{array}{cccc}6 & -0.531596 & -0.440159 & -0.271284 \\ 6 & 0.531596 & 0.440159 & -0.271284 \\ 6 & -0.498214 & -1.882546 & 0.06807 \\ 6 & -1.814191 & -2.411745 & -0.041229\end{array}$




\begin{tabular}{|c|c|c|c|}
\hline 6 & -2.703567 & -1.343515 & -0.495896 \\
\hline 6 & -1.943119 & -0.146052 & -0.607791 \\
\hline 6 & 0.498214 & 1.882546 & 0.06807 \\
\hline 6 & 1.814191 & 2.411745 & -0.041229 \\
\hline 6 & 2.703567 & 1.343515 & -0.495896 \\
\hline 6 & 1.943119 & 0.146052 & -0.607791 \\
\hline 6 & 0.513219 & -2.673203 & 0.615067 \\
\hline 6 & 0.245546 & -4.011673 & 0.969664 \\
\hline 6 & -1.055049 & -4.535911 & 0.797771 \\
\hline 6 & -2.085785 & -3.733865 & 0.311024 \\
\hline 6 & -4.053422 & -1.376509 & -0.844908 \\
\hline 6 & -4.654708 & -0.21335 & -1.334555 \\
\hline 6 & -3.900555 & 0.95533 & -1.503024 \\
\hline 6 & -2.546952 & 0.992778 & -1.156543 \\
\hline 6 & -0.513219 & 2.673203 & 0.615067 \\
\hline 6 & -0.245546 & 4.011673 & 0.969664 \\
\hline 6 & 1.055049 & 4.535911 & 0.797771 \\
\hline 6 & 2.085785 & 3.733865 & 0.311024 \\
\hline 6 & 4.053422 & 1.376509 & -0.844908 \\
\hline 6 & 4.654708 & 0.21335 & -1.334555 \\
\hline 6 & 3.900555 & -0.95533 & -1.503024 \\
\hline 6 & 2.546952 & -0.992778 & -1.156543 \\
\hline 1 & 1.510737 & -2.276486 & 0.792412 \\
\hline 1 & -1.247417 & -5.573765 & 1.074658 \\
\hline 1 & -3.097475 & -4.136346 & 0.225047 \\
\hline 1 & -4.628735 & -2.30085 & -0.756063 \\
\hline 1 & -5.711969 & -0.221836 & -1.609493 \\
\hline 1 & -1.974378 & 1.902154 & -1.332803 \\
\hline 1 & -1.510737 & 2.276486 & 0.792412 \\
\hline 1 & 1.247417 & 5.573765 & 1.074658 \\
\hline 1 & 3.097475 & 4.136346 & 0.225047 \\
\hline 1 & 4.628735 & 2.30085 & -0.756063 \\
\hline 1 & 5.711969 & 0.221836 & -1.609493 \\
\hline 1 & 4.37178 & -1.849091 & -1.918148 \\
\hline 1 & 1.974378 & -1.902154 & -1.332803 \\
\hline 6 & -1.288295 & 4.829353 & 1.508096 \\
\hline 6 & -2.174065 & 5.523282 & 1.963225 \\
\hline 1 & -2.957593 & 6.136128 & 2.367353 \\
\hline 1 & -4.37178 & 1.849091 & -1.918148 \\
\hline 6 & 1.288295 & -4.829353 & 1.508096 \\
\hline 6 & 2.174065 & -5.523282 & 1.963225 \\
\hline 1 & 2.957593 & -6.136128 & 2.367353 \\
\hline SAS & $=$ & 891.1 & $\AA$ \\
\hline Total dipole moment & $=$ & 0.76 & Debye \\
\hline
\end{tabular}


Chlorobenzene:

Table S21. B3LYP-D3/def2SVP (SCRF=SMD, Solvent=ChloroBenzene) optimized geometry of $\mathbf{1 E}$.

\begin{tabular}{|c|c|c|c|}
\hline 6 & -0.531611 & -0.440156 & -0.27149 \\
\hline 6 & 0.531611 & 0.440156 & -0.27149 \\
\hline 6 & -0.498205 & -1.882631 & 0.067752 \\
\hline 6 & -1.814282 & -2.411693 & -0.041012 \\
\hline 6 & -2.703745 & -1.343419 & -0.495317 \\
\hline 6 & -1.943277 & -0.146009 & -0.607505 \\
\hline 6 & 0.498205 & 1.882631 & 0.067752 \\
\hline 6 & 1.814282 & 2.411693 & -0.041012 \\
\hline 6 & 2.703745 & 1.343419 & -0.495317 \\
\hline 6 & 1.943277 & 0.146009 & -0.607505 \\
\hline 6 & 0.513328 & -2.673599 & 0.614102 \\
\hline 6 & 0.24557 & -4.012027 & 0.968824 \\
\hline 6 & -1.055197 & -4.536032 & 0.797521 \\
\hline 6 & -2.085985 & -3.733792 & 0.311221 \\
\hline 6 & -4.053737 & -1.376486 & -0.843757 \\
\hline 6 & -4.655238 & -0.213346 & -1.333159 \\
\hline 6 & -3.901157 & 0.955342 & -1.501883 \\
\hline 6 & -2.547421 & 0.992825 & -1.155936 \\
\hline 6 & -0.513328 & 2.673599 & 0.614102 \\
\hline 6 & -0.24557 & 4.012027 & 0.968824 \\
\hline 6 & 1.055197 & 4.536032 & 0.797521 \\
\hline 6 & 2.085985 & 3.733792 & 0.311221 \\
\hline 6 & 4.053737 & 1.376486 & -0.843757 \\
\hline 6 & 4.655238 & 0.213346 & -1.333159 \\
\hline 6 & 3.901157 & -0.955342 & -1.501883 \\
\hline 6 & 2.547421 & -0.992825 & -1.155936 \\
\hline 1 & 1.511092 & -2.277238 & 0.790635 \\
\hline 1 & -1.247611 & -5.573855 & 1.074332 \\
\hline 1 & -3.097755 & -4.136026 & 0.225474 \\
\hline 1 & -4.628918 & -2.300843 & -0.754663 \\
\hline 1 & -5.712553 & -0.221849 & -1.607672 \\
\hline 1 & -1.974979 & 1.902232 & -1.332257 \\
\hline 1 & -1.511092 & 2.277238 & 0.790635 \\
\hline 1 & 1.247611 & 5.573855 & 1.074332 \\
\hline 1 & 3.097755 & 4.136026 & 0.225474 \\
\hline 1 & 4.628918 & 2.300843 & -0.754663 \\
\hline 1 & 5.712553 & 0.221849 & -1.607672 \\
\hline 1 & 4.372596 & -1.849051 & -1.916748 \\
\hline 1 & 1.974979 & -1.902232 & -1.332257 \\
\hline 6 & -1.288495 & 4.829889 & 1.506629 \\
\hline 6 & -2.174454 & 5.523966 & 1.961161 \\
\hline 1 & -2.958169 & 6.137141 & 2.364397 \\
\hline 1 & -4.372596 & 1.849051 & -1.916748 \\
\hline 6 & 1.288495 & -4.829889 & 1.506629 \\
\hline
\end{tabular}




\begin{tabular}{cccc}
6 & 2.174454 & -5.523966 & 1.961161 \\
1 & 2.958169 & -6.137141 & 2.364397 \\
& & & \\
SAS & $=$ & 929.7 & $\AA$ \\
Total dipole moment & $=$ & 0.83 & Debye \\
\hline
\end{tabular}

Cyclohexane:

Table S22. B3LYP-D3/def2SVP (SCRF=SMD, Solvent=Cyclohexane) optimized geometry of $\mathbf{1 E}$.

\begin{tabular}{|c|c|c|c|}
\hline 6 & -0.531588 & -0.440151 & -0.271064 \\
\hline 6 & 0.531588 & 0.440151 & -0.271064 \\
\hline 6 & -0.498173 & -1.882621 & 0.068002 \\
\hline 6 & -1.814132 & -2.411796 & -0.041349 \\
\hline 6 & -2.703536 & -1.343513 & -0.495737 \\
\hline 6 & -1.943177 & -0.145994 & -0.607396 \\
\hline 6 & 0.498173 & 1.882621 & 0.068002 \\
\hline 6 & 1.814132 & 2.411796 & -0.041349 \\
\hline 6 & 2.703536 & 1.343513 & -0.495737 \\
\hline 6 & 1.943177 & 0.145994 & -0.607396 \\
\hline 6 & 0.513256 & -2.67344 & 0.614774 \\
\hline 6 & 0.245527 & -4.011973 & 0.969113 \\
\hline 6 & -1.055099 & -4.536174 & 0.797186 \\
\hline 6 & -2.085824 & -3.733973 & 0.310605 \\
\hline 6 & -4.053415 & -1.376612 & -0.844635 \\
\hline 6 & -4.654873 & -0.213392 & -1.333999 \\
\hline 6 & -3.900815 & 0.955431 & -1.502209 \\
\hline 6 & -2.547167 & 0.992926 & -1.155789 \\
\hline 6 & -0.513256 & 2.67344 & 0.614774 \\
\hline 6 & -0.245527 & 4.011973 & 0.969113 \\
\hline 6 & 1.055099 & 4.536174 & 0.797186 \\
\hline 6 & 2.085824 & 3.733973 & 0.310605 \\
\hline 6 & 4.053415 & 1.376612 & -0.844635 \\
\hline 6 & 4.654873 & 0.213392 & -1.333999 \\
\hline 6 & 3.900815 & -0.955431 & -1.502209 \\
\hline 6 & 2.547167 & -0.992926 & -1.155789 \\
\hline 1 & 1.510786 & -2.276805 & 0.791999 \\
\hline 1 & -1.247356 & -5.574035 & 1.073919 \\
\hline 1 & -3.097583 & -4.136155 & 0.224482 \\
\hline 1 & -4.628401 & -2.301121 & -0.755841 \\
\hline 1 & -5.712103 & -0.221889 & -1.608858 \\
\hline 1 & -1.974653 & 1.902376 & -1.331594 \\
\hline 1 & -1.510786 & 2.276805 & 0.791999 \\
\hline 1 & 1.247356 & 5.574035 & 1.073919 \\
\hline 1 & 3.097583 & 4.136155 & 0.224482 \\
\hline 1 & 4.628401 & 2.301121 & -0.755841 \\
\hline 1 & 5.712103 & 0.221889 & -1.608858 \\
\hline
\end{tabular}




\begin{tabular}{|cccc|}
\hline 1 & 4.372118 & -1.849234 & -1.91701 \\
\hline 1 & 1.974653 & -1.902376 & -1.331594 \\
\hline 6 & -1.288288 & 4.82981 & 1.507348 \\
6 & -2.174071 & 5.52386 & 1.96233 \\
\hline 1 & -2.957529 & 6.13672 & 2.366371 \\
\hline 1 & -4.372118 & 1.849234 & -1.91701 \\
\hline 6 & 1.288288 & -4.82981 & 1.507348 \\
6 & 2.174071 & -5.52386 & 1.96233 \\
\hline 1 & 2.957529 & -6.13672 & 2.366371 \\
& & & $\AA$ \\
\hline SAS & $=$ & 932.0 & $\AA$ \\
\hline Total dipole moment & $=$ & 0.75 & Debye \\
\hline
\end{tabular}

Toluene:

Table S23. B3LYP-D3/def2SVP (SCRF=SMD, Solvent=Toluene) optimized geometry of $\mathbf{1 E}$.

\begin{tabular}{|c|c|c|c|}
\hline 6 & -0.531594 & -0.440149 & -0.271176 \\
\hline 6 & 0.531594 & 0.440149 & -0.271176 \\
\hline 6 & -0.498197 & -1.88255 & 0.068059 \\
\hline 6 & -1.814156 & -2.411753 & -0.041301 \\
\hline 6 & -2.703539 & -1.343497 & -0.495842 \\
\hline 6 & -1.943121 & -0.146016 & -0.607626 \\
\hline 6 & 0.498197 & 1.88255 & 0.068059 \\
\hline 6 & 1.814156 & 2.411753 & -0.041301 \\
\hline 6 & 2.703539 & 1.343497 & -0.495842 \\
\hline 6 & 1.943121 & 0.146016 & -0.607626 \\
\hline 6 & 0.513233 & -2.673245 & 0.614984 \\
\hline 6 & 0.245555 & -4.011739 & 0.969445 \\
\hline 6 & -1.055017 & -4.535984 & 0.797477 \\
\hline 6 & -2.085747 & -3.733904 & 0.310793 \\
\hline 6 & -4.053394 & -1.376494 & -0.844812 \\
\hline 6 & -4.654719 & -0.213304 & -1.33431 \\
\hline 6 & -3.9006 & 0.955406 & -1.502669 \\
\hline 6 & -2.546993 & 0.992856 & -1.156228 \\
\hline 6 & -0.513233 & 2.673245 & 0.614984 \\
\hline 6 & -0.245555 & 4.011739 & 0.969445 \\
\hline 6 & 1.055017 & 4.535984 & 0.797477 \\
\hline 6 & 2.085747 & 3.733904 & 0.310793 \\
\hline 6 & 4.053394 & 1.376494 & -0.844812 \\
\hline 6 & 4.654719 & 0.213304 & -1.33431 \\
\hline 6 & 3.9006 & -0.955406 & -1.502669 \\
\hline 6 & 2.546993 & -0.992856 & -1.156228 \\
\hline 1 & 1.510735 & -2.27654 & 0.792414 \\
\hline 1 & -1.247339 & -5.573863 & 1.074262 \\
\hline 1 & -3.097439 & -4.136345 & 0.22474 \\
\hline 1 & -4.628641 & -2.300874 & -0.756033 \\
\hline
\end{tabular}




\begin{tabular}{|cccc}
\hline 1 & -5.711979 & -0.221776 & -1.609213 \\
\hline 1 & -1.974444 & 1.902256 & -1.332402 \\
1 & -1.510735 & 2.27654 & 0.792414 \\
1 & 1.247339 & 5.573863 & 1.074262 \\
1 & 3.097439 & 4.136345 & 0.22474 \\
\hline 1 & 4.628641 & 2.300874 & -0.756033 \\
1 & 5.711979 & 0.221776 & -1.609213 \\
\hline 1 & 4.371852 & -1.849198 & -1.917664 \\
1 & 1.974444 & -1.902256 & -1.332402 \\
6 & -1.28829 & 4.829443 & 1.507827 \\
6 & -2.174064 & 5.523381 & 1.962919 \\
\hline 1 & -2.957584 & 6.136233 & 2.367004 \\
1 & -4.371852 & 1.849198 & -1.917664 \\
\hline 6 & 1.28829 & -4.829443 & 1.507827 \\
6 & 2.174064 & -5.523381 & 1.962919 \\
\hline 1 & 2.957584 & -6.136233 & 2.367004 \\
\hline SAS & & & \\
\hline Total dipole moment & $=$ & 933.0 & $\AA$ \\
\hline
\end{tabular}

\subsection{2. $Z$-2,2'-Diethynyl-9,9'-bifluorenylidene}

Methanol:

Table S24. B3LYP-D3/def $2 S V P(S C R F=S M D$, Solvent=Methanol) optimized geometry of $\mathbf{1 Z}$.

\begin{tabular}{cccc}
6 & 0.645445 & -0.243876 & -0.668092 \\
6 & -0.645445 & 0.243876 & -0.668092 \\
6 & 1.604716 & -0.245894 & -1.794916 \\
6 & 2.828271 & -0.826269 & -1.358637 \\
6 & 2.665032 & -1.251393 & 0.031012 \\
6 & 1.362286 & -0.877665 & 0.463615 \\
6 & -1.362286 & 0.877665 & 0.463615 \\
6 & -2.665032 & 1.251393 & 0.031012 \\
\hline 6 & -2.828271 & 0.826269 & -1.358637 \\
6 & -1.604716 & 0.245894 & -1.794916 \\
6 & 1.552391 & 0.361825 & -3.055909 \\
6 & 2.672532 & 0.312353 & -3.890354 \\
6 & 3.854419 & -0.313395 & -3.472684 \\
6 & 3.94361 & -0.872112 & -2.194447 \\
6 & 3.526208 & -1.952522 & 0.874865 \\
6 & 3.090574 & -2.309913 & 2.149587 \\
6 & 1.779955 & -2.000413 & 2.576024 \\
6 & 0.912822 & -1.293656 & 1.717522 \\
6 & -0.912822 & 1.293656 & 1.717522 \\
6 & -1.779955 & 2.000413 & 2.576024 \\
\hline
\end{tabular}




\begin{tabular}{|c|c|c|c|}
\hline 6 & -3.090574 & 2.309913 & 2.149587 \\
\hline 6 & -3.526208 & 1.952522 & 0.874865 \\
\hline 6 & -3.94361 & 0.872112 & -2.194447 \\
\hline 6 & -3.854419 & 0.313395 & -3.472684 \\
\hline 6 & -2.672532 & -0.312353 & -3.890354 \\
\hline 6 & -1.552391 & -0.361825 & -3.055909 \\
\hline 1 & 0.658638 & 0.887831 & -3.388895 \\
\hline 1 & 2.625928 & 0.776652 & -4.878197 \\
\hline 1 & 4.718388 & -0.34334 & -4.140773 \\
\hline 1 & 4.879483 & -1.319127 & -1.851171 \\
\hline 1 & 4.526163 & -2.236898 & 0.539662 \\
\hline 1 & 3.751702 & -2.855394 & 2.825235 \\
\hline 1 & -0.105282 & -1.1007 & 2.050178 \\
\hline 1 & 0.105282 & 1.1007 & 2.050178 \\
\hline 1 & -3.751702 & 2.855394 & 2.825235 \\
\hline 1 & -4.526163 & 2.236898 & 0.539662 \\
\hline 1 & -4.879483 & 1.319127 & -1.851171 \\
\hline 1 & -4.718388 & 0.34334 & -4.140773 \\
\hline 1 & -2.625928 & -0.776652 & -4.878197 \\
\hline 1 & -0.658638 & -0.887831 & -3.388895 \\
\hline 6 & 1.326755 & -2.40783 & 3.869566 \\
\hline 6 & 0.941742 & -2.752081 & 4.967845 \\
\hline 1 & 0.599934 & -3.054913 & 5.939889 \\
\hline 6 & -1.326755 & 2.40783 & 3.869566 \\
\hline 6 & -0.941742 & 2.752081 & 4.967845 \\
\hline 1 & -0.599934 & 3.054913 & 5.939889 \\
\hline SAS & $=$ & 720.9 & $\AA$ \\
\hline Total dipole moment & $=$ & 1.96 & Debye \\
\hline
\end{tabular}

Ethanol:

Table S25. B3LYP-D3/def2SVP (SCRF=SMD, Solvent=Ethanol) optimized geometry of $\mathbf{1 Z}$.

\begin{tabular}{|cccc|}
\hline 6 & 0.645396 & -0.244029 & -0.667984 \\
\hline 6 & -0.645396 & 0.244029 & -0.667984 \\
6 & 1.604514 & -0.246163 & -1.795017 \\
6 & 2.82796 & -0.826967 & -1.359051 \\
6 & 2.664934 & -1.251986 & 0.03065 \\
\hline 6 & 1.362409 & -0.877909 & 0.463566 \\
6 & -1.362409 & 0.877909 & 0.463566 \\
\hline 6 & -2.664934 & 1.251986 & 0.03065 \\
\hline 6 & -2.82796 & 0.826967 & -1.359051 \\
6 & -1.604514 & 0.246163 & -1.795017 \\
6 & 1.552206 & 0.361861 & -3.055849 \\
6 & 2.672108 & 0.312132 & -3.890594 \\
6 & 3.853837 & -0.314142 & -3.473324 \\
\hline
\end{tabular}




\begin{tabular}{|c|c|c|c|}
\hline 6 & 3.94304 & -0.873051 & -2.195199 \\
\hline 6 & 3.526168 & -1.953115 & 0.874421 \\
\hline 6 & 3.090993 & -2.309906 & 2.149448 \\
\hline 6 & 1.78066 & -1.999861 & 2.576298 \\
\hline 6 & 0.913316 & -1.293409 & 1.717774 \\
\hline 6 & -0.913316 & 1.293409 & 1.717774 \\
\hline 6 & -1.78066 & 1.999861 & 2.576298 \\
\hline 6 & -3.090993 & 2.309906 & 2.149448 \\
\hline 6 & -3.526168 & 1.953115 & 0.874421 \\
\hline 6 & -3.94304 & 0.873051 & -2.195199 \\
\hline 6 & -3.853837 & 0.314142 & -3.473324 \\
\hline 6 & -2.672108 & -0.312132 & -3.890594 \\
\hline 6 & -1.552206 & -0.361861 & -3.055849 \\
\hline 1 & 0.658562 & 0.888338 & -3.388333 \\
\hline 1 & 2.625487 & 0.77675 & -4.878281 \\
\hline 1 & 4.717658 & -0.344267 & -4.141588 \\
\hline 1 & 4.878794 & -1.320415 & -1.852094 \\
\hline 1 & 4.525896 & -2.237802 & 0.538828 \\
\hline 1 & 3.752119 & -2.85521 & 2.825209 \\
\hline 1 & -0.104588 & -1.100171 & 2.050781 \\
\hline 1 & 0.104588 & 1.100171 & 2.050781 \\
\hline 1 & -3.752119 & 2.85521 & 2.825209 \\
\hline 1 & -4.525896 & 2.237802 & 0.538828 \\
\hline 1 & -4.878794 & 1.320415 & -1.852094 \\
\hline 1 & -4.717658 & 0.344267 & -4.141588 \\
\hline 1 & -2.625487 & -0.77675 & -4.878281 \\
\hline 1 & -0.658562 & -0.888338 & -3.388333 \\
\hline 6 & 1.328065 & -2.406051 & 3.870421 \\
\hline 6 & 0.943514 & -2.748858 & 4.969274 \\
\hline 1 & 0.601896 & -3.050188 & 5.941741 \\
\hline 6 & -1.328065 & 2.406051 & 3.870421 \\
\hline 6 & -0.943514 & 2.748858 & 4.969274 \\
\hline 1 & -0.601896 & 3.050188 & 5.941741 \\
\hline SAS & $=$ & 785.1 & $\AA$ \\
\hline Total dipole moment & $=$ & 1.89 & Debye \\
\hline
\end{tabular}

THF:

Table S26. B3LYP-D3/def2SVP (SCRF=SMD, Solvent=TetraHydroFuran) optimized geometry of $\mathbf{1 Z}$.

\begin{tabular}{cccc}
6 & 0.645399 & -0.244233 & -0.668075 \\
\hline 6 & -0.645399 & 0.244233 & -0.668075 \\
6 & 1.604633 & -0.246689 & -1.79535 \\
6 & 2.828042 & -0.827635 & -1.359405 \\
6 & 2.665091 & -1.25255 & 0.030371 \\
6 & 1.362734 & -0.87816 & 0.463487
\end{tabular}




\begin{tabular}{|c|c|c|c|}
\hline 6 & -1.362734 & 0.87816 & 0.463487 \\
\hline 6 & -2.665091 & 1.25255 & 0.030371 \\
\hline 6 & -2.828042 & 0.827635 & -1.359405 \\
\hline 6 & -1.604633 & 0.246689 & -1.79535 \\
\hline 6 & 1.55259 & 0.361154 & -3.056332 \\
\hline 6 & 2.672534 & 0.311356 & -3.891155 \\
\hline 6 & 3.854291 & -0.314957 & -3.473796 \\
\hline 6 & 3.943177 & -0.873876 & -2.195596 \\
\hline 6 & 3.526466 & -1.953717 & 0.874046 \\
\hline 6 & 3.091753 & -2.310141 & 2.149382 \\
\hline 6 & 1.781631 & -1.999528 & 2.576711 \\
\hline 6 & 0.914172 & -1.293155 & 1.718123 \\
\hline 6 & -0.914172 & 1.293155 & 1.718123 \\
\hline 6 & -1.781631 & 1.999528 & 2.576711 \\
\hline 6 & -3.091753 & 2.310141 & 2.149382 \\
\hline 6 & -3.526466 & 1.953717 & 0.874046 \\
\hline 6 & -3.943177 & 0.873876 & -2.195596 \\
\hline 6 & -3.854291 & 0.314957 & -3.473796 \\
\hline 6 & -2.672534 & -0.311356 & -3.891155 \\
\hline 6 & -1.55259 & -0.361154 & -3.056332 \\
\hline 1 & 0.658723 & 0.88706 & -3.388708 \\
\hline 1 & 2.625922 & 0.775987 & -4.878725 \\
\hline 1 & 4.718122 & -0.345074 & -4.141887 \\
\hline 1 & 4.878547 & -1.321418 & -1.851951 \\
\hline 1 & 4.525868 & -2.238406 & 0.537755 \\
\hline 1 & 3.752746 & -2.855343 & 2.825162 \\
\hline 1 & -0.103261 & -1.098938 & 2.051556 \\
\hline 1 & 0.103261 & 1.098938 & 2.051556 \\
\hline 1 & -3.752746 & 2.855343 & 2.825162 \\
\hline 1 & -4.525868 & 2.238406 & 0.537755 \\
\hline 1 & -4.878547 & 1.321418 & -1.851951 \\
\hline 1 & -4.718122 & 0.345074 & -4.141887 \\
\hline 1 & -2.625922 & -0.775987 & -4.878725 \\
\hline 1 & -0.658723 & -0.88706 & -3.388708 \\
\hline 6 & 1.329385 & -2.404803 & 3.871414 \\
\hline 6 & 0.944938 & -2.746586 & 4.970652 \\
\hline 1 & 0.603073 & -3.046845 & 5.94317 \\
\hline 6 & -1.329385 & 2.404803 & 3.871414 \\
\hline 6 & -0.944938 & 2.746586 & 4.970652 \\
\hline 1 & -0.603073 & 3.046845 & 5.94317 \\
\hline SAS & $=$ & 864.3 & $\AA$ \\
\hline Total dipole moment & $=$ & 1.78 & Debye \\
\hline
\end{tabular}

Benzene: 
Table S27. B3LYP-D3/def2SVP (SCRF=SMD, Solvent=Benzene) optimized geometry of 1 Z.

\begin{tabular}{|c|c|c|c|}
\hline 6 & 0.64545 & -0.244367 & -0.668082 \\
\hline 6 & -0.64545 & 0.244367 & -0.668082 \\
\hline 6 & 1.605026 & -0.246468 & -1.795357 \\
\hline 6 & 2.828504 & -0.827441 & -1.359395 \\
\hline 6 & 2.665351 & -1.252932 & 0.030302 \\
\hline 6 & 1.362902 & -0.878709 & 0.463365 \\
\hline 6 & -1.362902 & 0.878709 & 0.463365 \\
\hline 6 & -2.665351 & 1.252932 & 0.030302 \\
\hline 6 & -2.828504 & 0.827441 & -1.359395 \\
\hline 6 & -1.605026 & 0.246468 & -1.795357 \\
\hline 6 & 1.553259 & 0.361849 & -3.056183 \\
\hline 6 & 2.67336 & 0.312679 & -3.890956 \\
\hline 6 & 3.855216 & -0.313555 & -3.473597 \\
\hline 6 & 3.943812 & -0.873051 & -2.195567 \\
\hline 6 & 3.52657 & -1.954402 & 0.874014 \\
\hline 6 & 3.091775 & -2.311212 & 2.149266 \\
\hline 6 & 1.781569 & -2.000666 & 2.576651 \\
\hline 6 & 0.914293 & -1.294042 & 1.717959 \\
\hline 6 & -0.914293 & 1.294042 & 1.717959 \\
\hline 6 & -1.781569 & 2.000666 & 2.576651 \\
\hline 6 & -3.091775 & 2.311212 & 2.149266 \\
\hline 6 & -3.52657 & 1.954402 & 0.874014 \\
\hline 6 & -3.943812 & 0.873051 & -2.195567 \\
\hline 6 & -3.855216 & 0.313555 & -3.473597 \\
\hline 6 & -2.67336 & -0.312679 & -3.890956 \\
\hline 6 & -1.553259 & -0.361849 & -3.056183 \\
\hline 1 & 0.659226 & 0.887476 & -3.388401 \\
\hline 1 & 2.626784 & 0.777909 & -4.878254 \\
\hline 1 & 4.719292 & -0.343083 & -4.14142 \\
\hline 1 & 4.879137 & -1.320533 & -1.851759 \\
\hline 1 & 4.525992 & -2.238965 & 0.53771 \\
\hline 1 & 3.752661 & -2.856614 & 2.824961 \\
\hline 1 & -0.103043 & -1.099527 & 2.051317 \\
\hline 1 & 0.103043 & 1.099527 & 2.051317 \\
\hline 1 & -3.752661 & 2.856614 & 2.824961 \\
\hline 1 & -4.525992 & 2.238965 & 0.53771 \\
\hline 1 & -4.879137 & 1.320533 & -1.851759 \\
\hline 1 & -4.719292 & 0.343083 & -4.14142 \\
\hline 1 & -2.626784 & -0.777909 & -4.878254 \\
\hline 1 & -0.659226 & -0.887476 & -3.388401 \\
\hline 6 & 1.329032 & -2.406183 & 3.871356 \\
\hline 6 & 0.944093 & -2.748088 & 4.970412 \\
\hline 1 & 0.601381 & -3.048534 & 5.942534 \\
\hline 6 & -1.329032 & 2.406183 & 3.871356 \\
\hline 6 & -0.944093 & 2.748088 & 4.970412 \\
\hline
\end{tabular}




\begin{tabular}{cccc} 
SAS & $=$ & 879.3 & $\AA$ \\
Total dipole moment & $=$ & 1.67 & Debye \\
\hline
\end{tabular}

Chlorobenzene:

Table S28. B3LYP-D3/def2SVP (SCRF=SMD, Solvent=ChloroBenzene $)$ optimized geometry of $\mathbf{I Z}$.

\begin{tabular}{|c|c|c|c|}
\hline 6 & 0.645442 & -0.244419 & -0.668205 \\
\hline 6 & -0.645442 & 0.244419 & -0.668205 \\
\hline 6 & 1.604943 & -0.247058 & -1.795585 \\
\hline 6 & 2.828328 & -0.828169 & -1.359607 \\
\hline 6 & 2.665288 & -1.253188 & 0.03021 \\
\hline 6 & 1.362975 & -0.878585 & 0.463388 \\
\hline 6 & -1.362975 & 0.878585 & 0.463388 \\
\hline 6 & -2.665288 & 1.253188 & 0.03021 \\
\hline 6 & -2.828328 & 0.828169 & -1.359607 \\
\hline 6 & -1.604943 & 0.247058 & -1.795585 \\
\hline 6 & 1.553232 & 0.36062 & -3.056737 \\
\hline 6 & 2.673254 & 0.310795 & -3.891563 \\
\hline 6 & 3.855008 & -0.315526 & -3.474055 \\
\hline 6 & 3.943576 & -0.874478 & -2.195799 \\
\hline 6 & 3.526657 & -1.954487 & 0.87391 \\
\hline 6 & 3.092165 & -2.310779 & 2.149402 \\
\hline 6 & 1.782144 & -1.9998 & 2.577035 \\
\hline 6 & 0.914742 & -1.293365 & 1.718295 \\
\hline 6 & -0.914742 & 1.293365 & 1.718295 \\
\hline 6 & -1.782144 & 1.9998 & 2.577035 \\
\hline 6 & -3.092165 & 2.310779 & 2.149402 \\
\hline 6 & -3.526657 & 1.954487 & 0.87391 \\
\hline 6 & -3.943576 & 0.874478 & -2.195799 \\
\hline 6 & -3.855008 & 0.315526 & -3.474055 \\
\hline 6 & -2.673254 & -0.310795 & -3.891563 \\
\hline 6 & -1.553232 & -0.36062 & -3.056737 \\
\hline 1 & 0.659242 & 0.886035 & -3.389277 \\
\hline 1 & 2.626652 & 0.775372 & -4.879108 \\
\hline 1 & 4.718916 & -0.345675 & -4.141974 \\
\hline 1 & 4.878717 & -1.322164 & -1.851865 \\
\hline 1 & 4.525902 & -2.239228 & 0.537345 \\
\hline 1 & 3.75315 & -2.855991 & 2.825091 \\
\hline 1 & -0.102361 & -1.098367 & 2.051969 \\
\hline 1 & 0.102361 & 1.098367 & 2.051969 \\
\hline 1 & -3.75315 & 2.855991 & 2.825091 \\
\hline 1 & -4.525902 & 2.239228 & 0.537345 \\
\hline 1 & -4.878717 & 1.322164 & -1.851865 \\
\hline 1 & -4.718916 & 0.345675 & -4.141974 \\
\hline
\end{tabular}




\begin{tabular}{|cccc|}
\hline 1 & -2.626652 & -0.775372 & -4.879108 \\
\hline 1 & -0.659242 & -0.886035 & -3.389277 \\
6 & 1.329993 & -2.404525 & 3.872125 \\
6 & 0.945597 & -2.745692 & 4.971606 \\
1 & 0.603639 & -3.045382 & 5.944207 \\
\hline 6 & -1.329993 & 2.404525 & 3.872125 \\
6 & -0.945597 & 2.745692 & 4.971606 \\
\hline 1 & -0.603639 & 3.045382 & 5.944207 \\
& & & $\AA$ \\
\hline SAS & $=$ & 917.6 & Debye \\
\hline
\end{tabular}

Cyclohexane:

Table S29. B3LYP-D3/def2SVP (SCRF=SMD, Solvent=Cyclohexane) optimized geometry of $\mathbf{1 Z}$.

\begin{tabular}{|c|c|c|c|}
\hline 6 & 0.645407 & -0.24445 & -0.668208 \\
\hline 6 & -0.645407 & 0.24445 & -0.668208 \\
\hline 6 & 1.604921 & -0.246856 & -1.795579 \\
\hline 6 & 2.828354 & -0.827831 & -1.359554 \\
\hline 6 & 2.665276 & -1.253025 & 0.030209 \\
\hline 6 & 1.362917 & -0.878628 & 0.463313 \\
\hline 6 & -1.362917 & 0.878628 & 0.463313 \\
\hline 6 & -2.665276 & 1.253025 & 0.030209 \\
\hline 6 & -2.828354 & 0.827831 & -1.359554 \\
\hline 6 & -1.604921 & 0.246856 & -1.795579 \\
\hline 6 & 1.553197 & 0.361201 & -3.056531 \\
\hline 6 & 2.673318 & 0.311789 & -3.891318 \\
\hline 6 & 3.855166 & -0.314465 & -3.473841 \\
\hline 6 & 3.94368 & -0.873744 & -2.195683 \\
\hline 6 & 3.526647 & -1.954315 & 0.873907 \\
\hline 6 & 3.092045 & -2.310777 & 2.149349 \\
\hline 6 & 1.781908 & -2.000003 & 2.576836 \\
\hline 6 & 0.914495 & -1.293601 & 1.718095 \\
\hline 6 & -0.914495 & 1.293601 & 1.718095 \\
\hline 6 & -1.781908 & 2.000003 & 2.576836 \\
\hline 6 & -3.092045 & 2.310777 & 2.149349 \\
\hline 6 & -3.526647 & 1.954315 & 0.873907 \\
\hline 6 & -3.94368 & 0.873744 & -2.195683 \\
\hline 6 & -3.855166 & 0.314465 & -3.473841 \\
\hline 6 & -2.673318 & -0.311789 & -3.891318 \\
\hline 6 & -1.553197 & -0.361201 & -3.056531 \\
\hline 1 & 0.659107 & 0.886643 & -3.388755 \\
\hline 1 & 2.626771 & 0.776827 & -4.87865 \\
\hline 1 & 4.719197 & -0.344162 & -4.141633 \\
\hline 1 & 4.878815 & -1.321298 & -1.851561 \\
\hline 1 & 4.525934 & -2.238865 & 0.537304 \\
\hline
\end{tabular}




\begin{tabular}{cccc}
\hline 1 & 3.752884 & -2.855988 & 2.82516 \\
1 & -0.102691 & -1.098771 & 2.051596 \\
1 & 0.102691 & 1.098771 & 2.051596 \\
1 & -3.752884 & 2.855988 & 2.82516 \\
\hline 1 & -4.525934 & 2.238865 & 0.537304 \\
1 & -4.878815 & 1.321298 & -1.851561 \\
1 & -4.719197 & 0.344162 & -4.141633 \\
1 & -2.626771 & -0.776827 & -4.87865 \\
\hline 1 & -0.659107 & -0.886643 & -3.388755 \\
6 & 1.329589 & -2.404985 & 3.871809 \\
6 & 0.944805 & -2.746353 & 4.971108 \\
\hline 1 & 0.602172 & -3.046325 & 5.943323 \\
\hline 6 & -1.329589 & 2.404985 & 3.871809 \\
6 & -0.944805 & 2.746353 & 4.971108 \\
\hline 1 & -0.602172 & 3.046325 & 5.943323 \\
& $=$ & & $\AA$ \\
SAS & $=$ & 919.8 & \\
Total dipole moment & $=$ & 1.65 & Debye \\
\hline
\end{tabular}

Toluene:

Table S30. B3LYP-D3/def2SVP (SCRF=SMD, Solvent=Toluene) optimized geometry of $\mathbf{1 Z}$.

\begin{tabular}{|c|c|c|c|}
\hline 6 & 0.645425 & -0.244408 & -0.668166 \\
\hline 6 & -0.645425 & 0.244408 & -0.668166 \\
\hline 6 & 1.604961 & -0.246645 & -1.79547 \\
\hline 6 & 2.828409 & -0.827644 & -1.359492 \\
\hline 6 & 2.665297 & -1.252968 & 0.030241 \\
\hline 6 & 1.362882 & -0.878675 & 0.463307 \\
\hline 6 & -1.362882 & 0.878675 & 0.463307 \\
\hline 6 & -2.665297 & 1.252968 & 0.030241 \\
\hline 6 & -2.828409 & 0.827644 & -1.359492 \\
\hline 6 & -1.604961 & 0.246645 & -1.79547 \\
\hline 6 & 1.553206 & 0.361565 & -3.056338 \\
\hline 6 & 2.673282 & 0.312235 & -3.891129 \\
\hline 6 & 3.855098 & -0.314048 & -3.473753 \\
\hline 6 & 3.943685 & -0.873421 & -2.195678 \\
\hline 6 & 3.526576 & -1.954285 & 0.873999 \\
\hline 6 & 3.091882 & -2.31084 & 2.149349 \\
\hline 6 & 1.781711 & -2.000215 & 2.576751 \\
\hline 6 & 0.914349 & -1.293809 & 1.717986 \\
\hline 6 & -0.914349 & 1.293809 & 1.717986 \\
\hline 6 & -1.781711 & 2.000215 & 2.576751 \\
\hline 6 & -3.091882 & 2.31084 & 2.149349 \\
\hline 6 & -3.526576 & 1.954285 & 0.873999 \\
\hline 6 & -3.943685 & 0.873421 & -2.195678 \\
\hline 6 & -3.855098 & 0.314048 & -3.473753 \\
\hline
\end{tabular}




\begin{tabular}{|c|c|c|c|}
\hline 6 & -2.673282 & -0.312235 & -3.891129 \\
\hline 6 & -1.553206 & -0.361565 & -3.056338 \\
\hline 1 & 0.659209 & 0.88724 & -3.388556 \\
\hline 1 & 2.626717 & 0.777381 & -4.878455 \\
\hline 1 & 4.719139 & -0.343704 & -4.141598 \\
\hline 1 & 4.878961 & -1.320946 & -1.851815 \\
\hline 1 & 4.525964 & -2.238877 & 0.537638 \\
\hline 1 & 3.752792 & -2.856078 & 2.825136 \\
\hline 1 & -0.102965 & -1.099277 & 2.051381 \\
\hline 1 & 0.102965 & 1.099277 & 2.051381 \\
\hline 1 & -3.752792 & 2.856078 & 2.825136 \\
\hline 1 & -4.525964 & 2.238877 & 0.537638 \\
\hline 1 & -4.878961 & 1.320946 & -1.851815 \\
\hline 1 & -4.719139 & 0.343704 & -4.141598 \\
\hline 1 & -2.626717 & -0.777381 & -4.878455 \\
\hline 1 & -0.659209 & -0.88724 & -3.388556 \\
\hline 6 & 1.329313 & -2.405354 & 3.871603 \\
\hline 6 & 0.944503 & -2.746856 & 4.970822 \\
\hline 1 & 0.601889 & -3.046942 & 5.94307 \\
\hline 6 & -1.329313 & 2.405354 & 3.871603 \\
\hline 6 & -0.944503 & 2.746856 & 4.970822 \\
\hline 1 & -0.601889 & 3.046942 & 5.94307 \\
\hline SAS & $=$ & 920.8 & $\AA$ \\
\hline Total dipole moment & $=$ & 1.67 & Debye \\
\hline
\end{tabular}

\subsubsection{E-3,3'-Dibromo-9,9'-bifluorenylidene}

Methanol:

Table S31. B3LYP-D3/def2SVP (SCRF=SMD, Solvent=Methanol) optimized geometry of S1Z.

\begin{tabular}{cccc}
1 & 3.388514 & 2.035885 & -1.124702 \\
\hline 35 & 3.21209 & 4.92748 & -0.495725 \\
6 & 2.01214 & 3.465122 & -0.265282 \\
6 & 2.408548 & 2.184174 & -0.669486 \\
6 & 0.74638 & 3.699538 & 0.279677 \\
\hline 1 & 0.435943 & 4.710965 & 0.545096 \\
6 & 1.543577 & 1.100847 & -0.498818 \\
\hline 1 & 1.854411 & 0.119349 & -0.854409 \\
6 & -0.10774 & 2.611705 & 0.447464 \\
6 & 0.292766 & 1.291589 & 0.101192 \\
\hline 1 & -1.940219 & 4.590329 & 1.539764 \\
6 & -1.496195 & 2.565593 & 0.910884 \\
6 & -2.305329 & 3.56461 & 1.449226 \\
\hline 1 & -3.610158 & -0.336668 & -0.911108 \\
6 & -0.855441 & 0.378647 & 0.28737 \\
\hline
\end{tabular}




\begin{tabular}{|c|c|c|c|}
\hline 6 & -3.297666 & -1.379935 & -0.929688 \\
\hline 1 & -5.150105 & -2.035449 & -1.828905 \\
\hline 6 & -4.166111 & -2.333828 & -1.465216 \\
\hline 6 & -1.959922 & 1.226705 & 0.789303 \\
\hline 6 & -0.890018 & -0.979309 & 0.038651 \\
\hline 6 & -3.586754 & 3.228726 & 1.896554 \\
\hline 6 & -2.041536 & -1.768886 & -0.448257 \\
\hline 35 & -4.972747 & -4.961845 & -2.268004 \\
\hline 6 & -3.768109 & -3.674172 & -1.545356 \\
\hline 1 & -4.234999 & 4.000236 & 2.318577 \\
\hline 6 & 0.217709 & -1.948324 & 0.196664 \\
\hline 6 & -1.640056 & -3.125299 & -0.596491 \\
\hline 6 & -3.222585 & 0.89675 & 1.298183 \\
\hline 6 & -4.031815 & 1.901947 & 1.835323 \\
\hline 6 & -2.49763 & -4.084408 & -1.131026 \\
\hline 6 & 1.485733 & -1.818083 & 0.777441 \\
\hline 6 & -0.247174 & -3.244111 & -0.160077 \\
\hline 1 & 1.840704 & -0.854553 & 1.141082 \\
\hline 6 & 2.298283 & -2.947024 & 0.915455 \\
\hline 6 & 0.565303 & -4.3677 & -0.018747 \\
\hline 1 & -2.186446 & -5.12368 & -1.244523 \\
\hline 1 & -3.575759 & -0.133733 & 1.29984 \\
\hline 6 & 1.851512 & -4.210055 & 0.506405 \\
\hline 1 & -5.021134 & 1.644738 & 2.220867 \\
\hline 1 & 3.291675 & -2.841538 & 1.357721 \\
\hline 1 & 0.199282 & -5.359493 & -0.294388 \\
\hline 1 & 2.502398 & -5.079953 & 0.621197 \\
\hline SAS & $=$ & 714.5 & $\AA$ \\
\hline Total dipole moment & $=$ & 1.09 & Debye \\
\hline
\end{tabular}

Benzene:

Table S32. B3LYP-D3/def2SVP (SCRF=SMD, Solvent=Benzene) optimized geometry of $\boldsymbol{S 1 Z}$.

\begin{tabular}{|cccc|}
\hline 1 & 3.38822 & 2.039506 & -1.127049 \\
\hline 35 & 3.212176 & 4.929984 & -0.495272 \\
6 & 2.012554 & 3.467232 & -0.265426 \\
6 & 2.408585 & 2.186392 & -0.670977 \\
\hline 6 & 0.746924 & 3.7006 & 0.280509 \\
\hline 1 & 0.43687 & 4.711721 & 0.546938 \\
\hline 6 & 1.543642 & 1.102867 & -0.500814 \\
\hline 1 & 1.853858 & 0.121377 & -0.856435 \\
6 & -0.107299 & 2.612599 & 0.447714 \\
6 & 0.292805 & 1.292717 & 0.099903 \\
\hline 1 & -1.93811 & 4.589813 & 1.543271 \\
\hline 6 & -1.495679 & 2.566114 & 0.91182 \\
\hline
\end{tabular}




\begin{tabular}{|c|c|c|c|}
\hline 6 & -2.304435 & 3.564737 & 1.451907 \\
\hline 1 & -3.609628 & -0.337846 & -0.913727 \\
\hline 6 & -0.855393 & 0.379171 & 0.285614 \\
\hline 6 & -3.297754 & -1.381117 & -0.932269 \\
\hline 1 & -5.14984 & -2.038003 & -1.832384 \\
\hline 6 & -4.166166 & -2.335375 & -1.467395 \\
\hline 6 & -1.959899 & 1.227445 & 0.788672 \\
\hline 6 & -0.890083 & -0.979176 & 0.036824 \\
\hline 6 & -3.585894 & 3.229042 & 1.89955 \\
\hline 6 & -2.041588 & -1.769484 & -0.44986 \\
\hline 35 & -4.972833 & -4.964349 & -2.268463 \\
\hline 6 & -3.768528 & -3.676096 & -1.546232 \\
\hline 1 & -4.233424 & 4.000169 & 2.323106 \\
\hline 6 & 0.217679 & -1.948792 & 0.195813 \\
\hline 6 & -1.640495 & -3.126225 & -0.596568 \\
\hline 6 & -3.222815 & 0.897708 & 1.297361 \\
\hline 6 & -4.031611 & 1.90245 & 1.83639 \\
\hline 6 & -2.498168 & -4.085699 & -1.130617 \\
\hline 6 & 1.485953 & -1.818687 & 0.776332 \\
\hline 6 & -0.247682 & -3.244931 & -0.159379 \\
\hline 1 & 1.841152 & -0.854458 & 1.137297 \\
\hline 6 & 2.298089 & -2.947873 & 0.916277 \\
\hline 6 & 0.564435 & -4.36877 & -0.016274 \\
\hline 1 & -2.187356 & -5.125043 & -1.243056 \\
\hline 1 & -3.576244 & -0.13248 & 1.296271 \\
\hline 6 & 1.85068 & -4.211413 & 0.509105 \\
\hline 1 & -5.020711 & 1.644909 & 2.221978 \\
\hline 1 & 3.291262 & -2.842092 & 1.358705 \\
\hline 1 & 0.197207 & -5.360258 & -0.290902 \\
\hline 1 & 2.500865 & -5.081498 & 0.625474 \\
\hline SAS & $=$ & 881.5 & $\AA$ \\
\hline Total dipole moment & $=$ & 0.93 & Debye \\
\hline
\end{tabular}

\subsection{4. $Z$-3,3'-Dibromo-9,9'-bifluorenylidene}

Methanol:

Table S33. B3LYP-D3/def2SVP (SCRF=SMD, Solvent=Methanol) optimized geometry of S1Z.

\begin{tabular}{|cccc|}
1 & 3.451932 & 2.055137 & -1.061478 \\
6 & 2.085264 & 3.491651 & -0.2012 \\
6 & 2.468061 & 2.208304 & -0.611915 \\
6 & 0.81257 & 3.710699 & 0.334631 \\
1 & 0.49645 & 4.718931 & 0.612386 \\
\hline 6 & 1.604152 & 1.119265 & -0.465737 \\
\hline 1 & 1.910631 & 0.139071 & -0.828626
\end{tabular}




\begin{tabular}{|c|c|c|c|}
\hline 6 & -0.051155 & 2.627095 & 0.484021 \\
\hline 6 & 0.348206 & 1.310335 & 0.124329 \\
\hline 1 & -1.888475 & 4.597706 & 1.585349 \\
\hline 6 & -1.443916 & 2.575439 & 0.933652 \\
\hline 6 & -2.249655 & 3.574286 & 1.475969 \\
\hline 1 & -3.545782 & -0.296133 & -0.9473 \\
\hline 6 & -0.804029 & 0.396141 & 0.290841 \\
\hline 6 & -3.242425 & -1.342289 & -0.959363 \\
\hline 1 & -5.091801 & -1.983636 & -1.875258 \\
\hline 6 & -4.113931 & -2.289667 & -1.501545 \\
\hline 6 & -1.910896 & 1.239682 & 0.790533 \\
\hline 6 & -0.84176 & -0.960024 & 0.032923 \\
\hline 6 & -3.533854 & 3.224924 & 1.903748 \\
\hline 6 & -1.994296 & -1.740998 & -0.465223 \\
\hline 35 & -4.934472 & -4.912148 & -2.308041 \\
\hline 6 & -3.726155 & -3.633339 & -1.576138 \\
\hline 35 & -4.668544 & 4.568614 & 2.637258 \\
\hline 6 & 0.258342 & -1.936591 & 0.197894 \\
\hline 6 & -1.602153 & -3.100547 & -0.608925 \\
\hline 6 & -3.178502 & 0.91064 & 1.28639 \\
\hline 6 & -3.9955 & 1.904775 & 1.82973 \\
\hline 6 & -2.462676 & -4.053253 & -1.150099 \\
\hline 6 & 1.523745 & -1.815341 & 0.786231 \\
\hline 6 & -0.213771 & -3.229218 & -0.161196 \\
\hline 1 & 1.884498 & -0.853623 & 1.148661 \\
\hline 6 & 2.326259 & -2.950526 & 0.931273 \\
\hline 6 & 0.588864 & -4.358968 & -0.012943 \\
\hline 1 & -2.158839 & -5.095089 & -1.259929 \\
\hline 1 & -3.539308 & -0.117124 & 1.274778 \\
\hline 6 & 1.872463 & -4.210708 & 0.521136 \\
\hline 1 & -4.988297 & 1.653316 & 2.204789 \\
\hline 1 & 3.317713 & -2.85206 & 1.379482 \\
\hline 1 & 0.21705 & -5.348151 & -0.290223 \\
\hline 1 & 2.515779 & -5.085482 & 0.641468 \\
\hline 1 & 2.775842 & 4.329487 & -0.322442 \\
\hline SAS & $=$ & 714.7 & $\AA$ \\
\hline Total dipole moment & $=$ & 2.74 & Debye \\
\hline
\end{tabular}

Benzene:

Table S34. B3LYP-D3/def2SVP (SCRF=SMD, Solvent=Benzene) optimized geometry of $\boldsymbol{S 1 Z}$.

\begin{tabular}{|llll|}
\hline 1 & -1.852515 & 5.342759 & -2.023316 \\
\hline 6 & -3.466369 & 3.936543 & -1.723429 \\
6 & -2.13155 & 4.355155 & -1.648992 \\
6 & -3.829833 & 2.658051 & -1.289321 \\
\hline
\end{tabular}




\begin{tabular}{|c|c|c|c|}
\hline 1 & -4.861525 & 2.313074 & -1.389005 \\
\hline 6 & -1.145796 & 3.521723 & -1.11251 \\
\hline 1 & -0.109128 & 3.855158 & -1.100749 \\
\hline 6 & -2.85025 & 1.82464 & -0.751869 \\
\hline 6 & -1.503012 & 2.260582 & -0.618247 \\
\hline 1 & -5.036767 & -0.066967 & -0.413369 \\
\hline 6 & -2.927568 & 0.433622 & -0.300006 \\
\hline 6 & -4.033454 & -0.399591 & -0.144656 \\
\hline 1 & 0.483887 & -1.586972 & -1.003487 \\
\hline 6 & -0.679612 & 1.133818 & -0.123236 \\
\hline 6 & 1.456601 & -1.254802 & -0.643345 \\
\hline 1 & 2.433648 & -3.084561 & -1.251425 \\
\hline 6 & 2.558209 & -2.09899 & -0.801534 \\
\hline 6 & -1.617863 & 0.003835 & 0.050561 \\
\hline 6 & 0.679612 & 1.133819 & 0.123227 \\
\hline 6 & -3.828603 & -1.673958 & 0.39245 \\
\hline 6 & 1.617864 & 0.003837 & -0.050567 \\
\hline 35 & 5.316553 & -2.845126 & -0.605719 \\
\hline 6 & 3.828606 & -1.673954 & -0.392451 \\
\hline 35 & -5.316548 & -2.845132 & 0.605723 \\
\hline 6 & 1.503008 & 2.260583 & 0.618244 \\
\hline 6 & 2.927567 & 0.433625 & 0.300005 \\
\hline 6 & -1.456597 & -1.254806 & 0.643335 \\
\hline 6 & -2.558204 & -2.098995 & 0.801528 \\
\hline 6 & 4.033454 & -0.399588 & 0.144658 \\
\hline 6 & 1.14579 & 3.521723 & 1.112507 \\
\hline 6 & 2.850246 & 1.824642 & 0.75187 \\
\hline 1 & 0.109122 & 3.855158 & 1.100742 \\
\hline 6 & 2.131541 & 4.355155 & 1.648995 \\
\hline 6 & 3.829827 & 2.658053 & 1.289328 \\
\hline 1 & 5.036767 & -0.066963 & 0.413376 \\
\hline 1 & -0.483881 & -1.586976 & 1.003472 \\
\hline 6 & 3.46636 & 3.936543 & 1.723436 \\
\hline 1 & -2.43364 & -3.084567 & 1.251415 \\
\hline 1 & 1.852505 & 5.342758 & 2.023318 \\
\hline 1 & 4.861518 & 2.313076 & 1.389015 \\
\hline 1 & 4.22223 & 4.603224 & 2.144795 \\
\hline 1 & -4.22224 & 4.603224 & -2.144783 \\
\hline SAS & $=$ & 881.1 & $\AA ̊$ \\
\hline Total dipole moment & $=$ & 2.52 & Debye \\
\hline
\end{tabular}

\section{References}

(1) Majer, V.; Svoboda, V.; Hála, S.; Pick, J., Temperature dependence of heats of vaporization of saturated hydrocarbons $\mathrm{C} 5-\mathrm{C} 8$; Experimental data and an estimation method Collect. Czech. Chem. C. 1979, 44, 637-651. 
(2) Eubank, P. T.; Cediel, L. E.; Holste, J. C.; Hall, K. R., Enthalpies for toluene and methylcyclohexane in the fluid state, J. Chem. Eng. Data 1984, 29, 389-393.

(3) Gaw, W. J.; Swinton, F. L. Thermodynamic properties of binary systems containing hexafluorobenzene. Part 4.-Excess Gibbs free energies of the three systems hexafluorobenzene + benzene, toluene, and p-xylene T. Faraday Soc. 1968, 64, 2023-2034.

(4) Viton, C.; Chavret, M.; Jose, J., Enthalpies of vaporization of normal alkanes from nonane to pentadecane at temperatures from 298 to $359 \mathrm{~K}$ ELDATA: Int. Electron. J. Phys. Chem. Data 1996, 2, 103.

(5) Stephenson, R. M.; Malanowski, S., Handbook of the Thermodynamics of Organic Compounds 1987.

(6) Dong, J.-Q.; Lin, R.-S.; Yen, W.-H., Heats of vaporization and gaseous molar heat capacities of ethanol and the binary mixture of ethanol and benzene Can. J. Chem. 1988, 66, 783-790.

(7) Dreyer, R.; Martin, W.; von Weber, U., Die Sättigungsdampfdrucke von Benzol, Toluol, Äthylbenzol, Styrol, Cumol und Brombenzol zwischen 10 und 760 Torr J. Prakt. Chem. 1955, $1,324-328$

(8) Majer, V.; Svoboda, V., Enthalpies of Vaporization of Organic Compounds: A Critical Review and Data Compilation, Blackwell Scientific Publications, Oxford, 1985, 300.

(9) Majer, V.; Svoboda, V.; Lencka, M., Enthalpies of vaporization and cohesive energies of pyridine and isomeric methylpyridines $J$. Chem. Thermodyn. 1984, 16, 1019-1024.

(10) Zaraiskii, A.P., Zh. Fiz. Khim., 1985, 59, 2087.

(11) Vercher, E.; Vázquez, M. I.; Martínez-Andreu, A., Isobaric Vapor-Liquid Equilibria for Water + Acetic Acid + Lithium Acetate J. Chem. Eng. Data 2001, 46, 1584-1588.

(12) Cervenkova, I.; Boublik, T., Vapor pressure, refractive indexes and densities at 20.0.degree.C, and vapor-liquid equilibrium at $101.325 \mathrm{kPa}$ in the tert-amyl methyl ether-methanol system J. Chem. Eng. Data 1984, 29, 425-427.

(13) Kawata, S.; Furudate, J.; Kimura, T.; Minaki, H.; Saito, A.; Katagiri, H.; Pu, Y.-J.; Controlling the excited-state energy levels of 9,9'-bifluorenylidene derivatives by twisting their structure to attaining singlet fission character in organic photovoltaics J. Mater. Chem. C, 2017, $5,4909-4914$.

(14) Krause, L.; Herbst-Irmer, R.; Sheldrick, G. M.; Stalke, D. J. Appl. Cryst. 2015, 48, 3-10.

(15) Sheldrick, G. M., SHELXT - Integrated space-group and crystal-structure determination Acta Cryst. A 2015, 71, 3-8.

(16) Sheldrick, G. M., Crystal structure refinement with SHELXL Acta Cryst. C 2015, 71, 38.

(17) https://www.ccdc.cam.ac.uk/structures/ 